Internet Engineering Task Force (IETF)

Request for Comments: 5934

R. Housley

Category: Standards Track

ISSN : 2070-1721

Vigil Security, LLC

S. Ashmore

National Security Agency

C. Wallace

Cygnacom Solutions

August 2010

Trust Anchor Management Protocol (TAMP)

Abstract

This document describes a transport independent protocol for the management of trust anchors (TAs) and community identifiers stored in a trust anchor store. The protocol makes use of the cryptographic Message Syntax (CMS), and a digital signature is used to provide integrity protection and data origin authentication. The protocol can be used to manage trust anchor stores containing trust anchors represented as Certificate, TBSCertificate, or TrustAnchorInfo objects.

Status of This Memo

This is an Internet Standards Track document.

This document is a product of the Internet Engineering Task Force (IETF). It represents the consensus of the IETF community. It has received public review and has been approved for publication by the Internet Engineering Steering Group (IESG). Further information on Internet standards is available in Section 2 of RFC 5741.

Information about the current status of this document, any errata, and how to provide feedback on it may be obtained at http: //wWw.rfc-editor.org/info/rfc5934. 
Copyright Notice

Copyright (c) 2010 IETF Trust and the persons identified as the document authors. All rights reserved.

This document is subject to BCP 78 and the IETF Trust's Legal Provisions Relating to IETF Documents (http://trustee.ietf.org/license-info) in effect on the date of publication of this document. Please review these documents carefully, as they describe your rights and restrictions with respect to this document. Code Components extracted from this document must include Simplified BSD License text as described in section $4 . e$ of the Trust Legal Provisions and are provided without warranty as described in the Simplified BSD License.

This document may contain material from IETF Documents or IETF Contributions published or made publicly available before November 10, 2008. The person(s) controlling the copyright in some of this material may not have granted the IETF Trust the right to allow modifications of such material outside the IETF Standards Process. Without obtaining an adequate license from the person(s) controlling the copyright in such materials, this document may not be modified outside the IETF standards Process, and derivative works of it may not be created outside the IETF Standards Process, except to format it for publication as an RFC or to translate it into languages other than English. 
Table of Contents

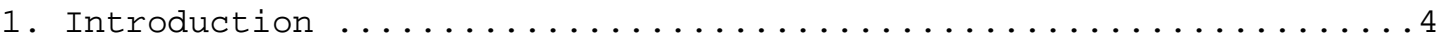

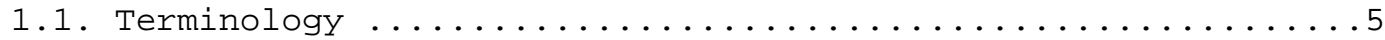

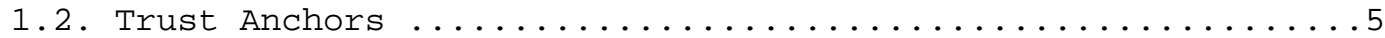

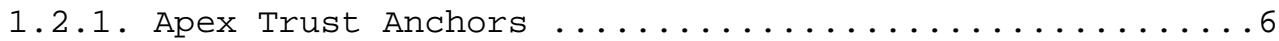

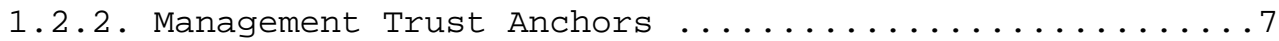

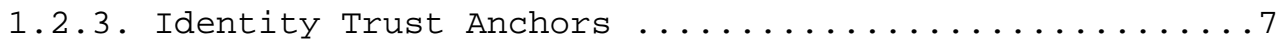

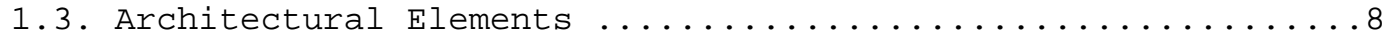

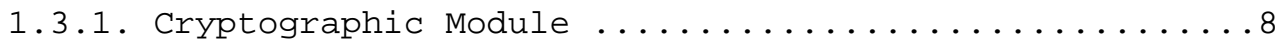

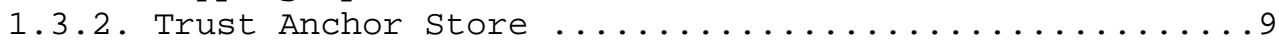

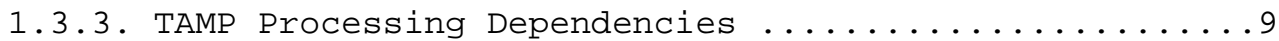

1.3.4. Application-Specific Protocol Processing .........10

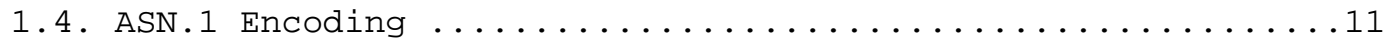

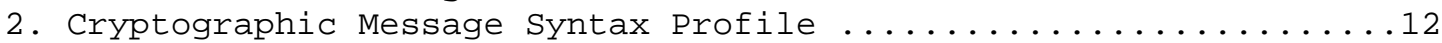

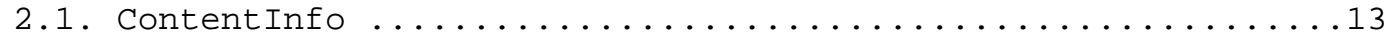

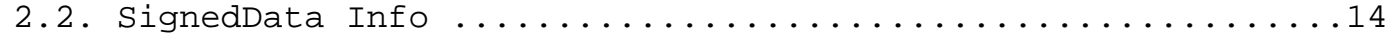

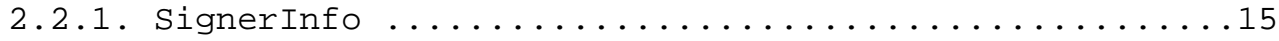

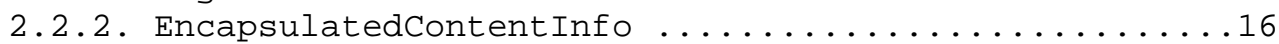

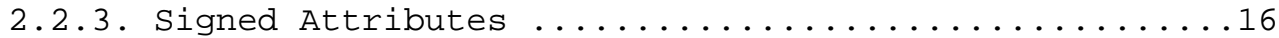

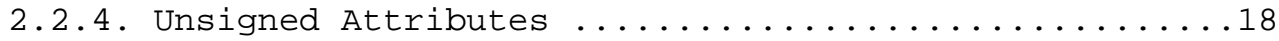

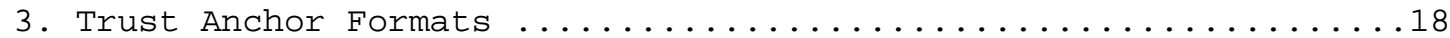

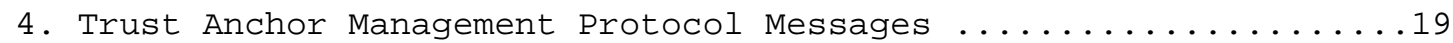

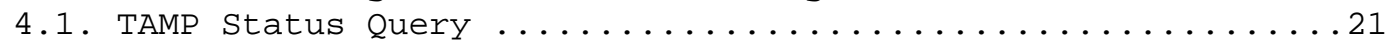

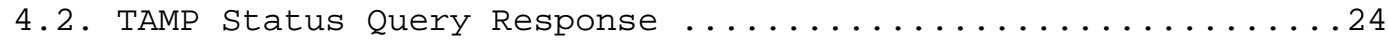

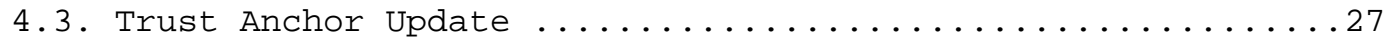

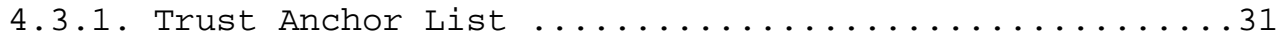

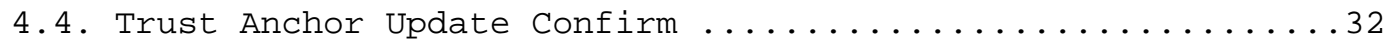

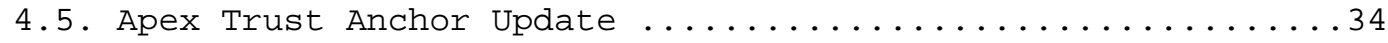

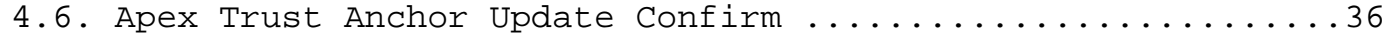

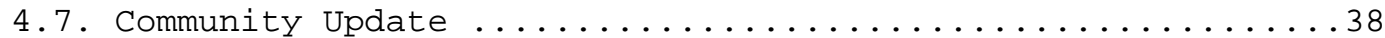

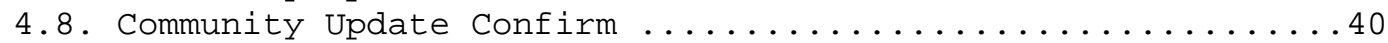

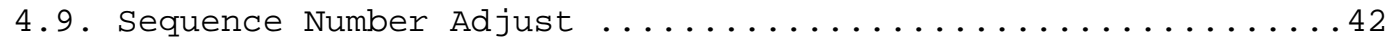

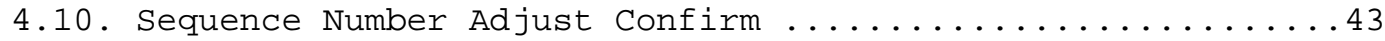

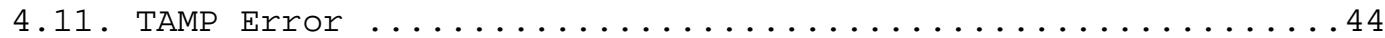

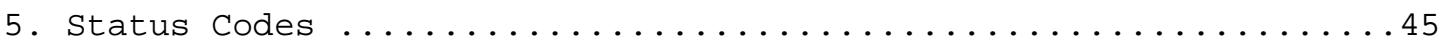

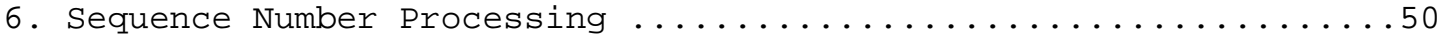

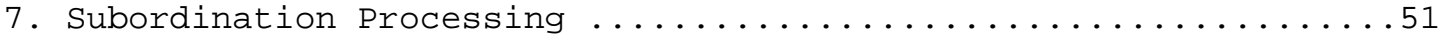

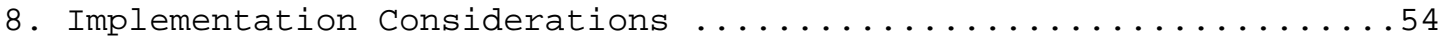

9. Wrapped Apex Contingency Key Certificate Extension .........54

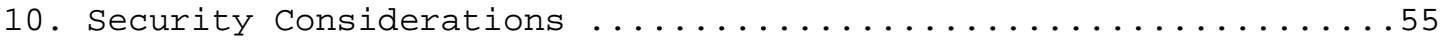

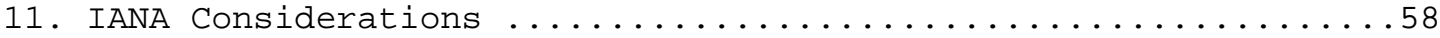

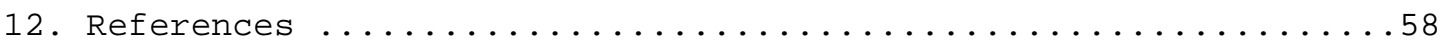

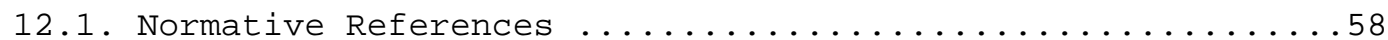

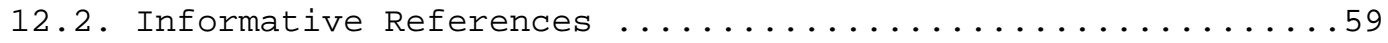




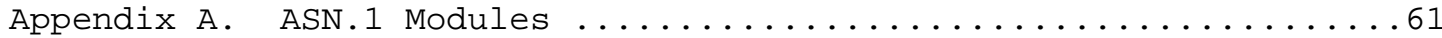

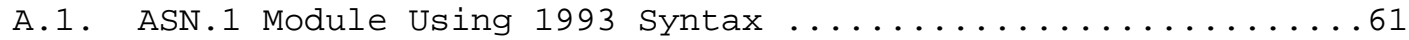

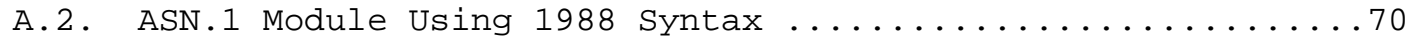

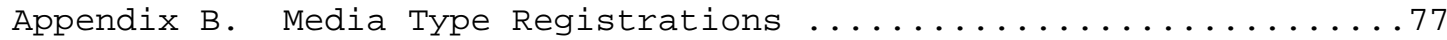

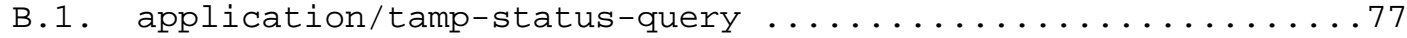

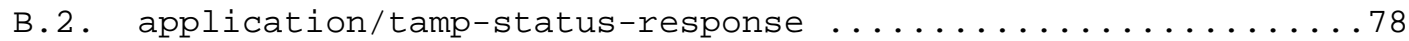

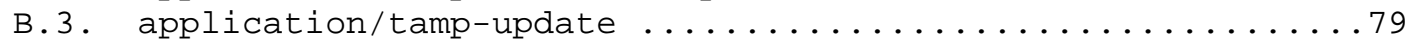

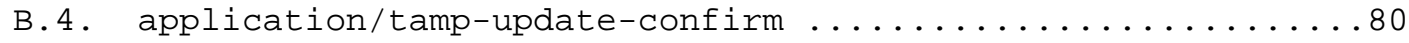

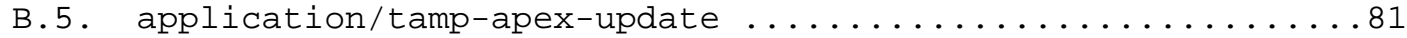

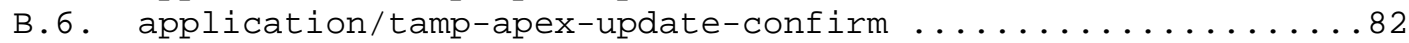

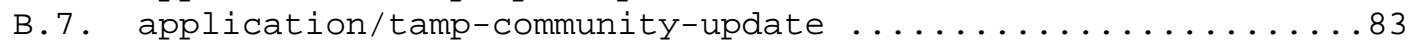

B.8. application/tamp-community-update-confirm ..........84

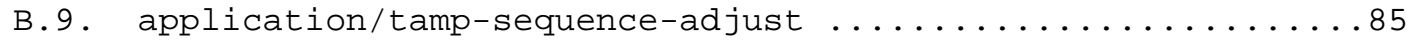

B.10. application/tamp-sequence-adjust-confirm ..........86

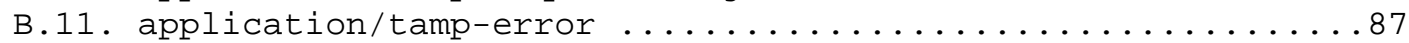

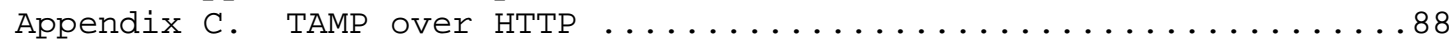

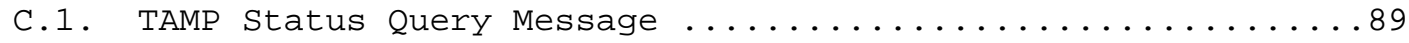

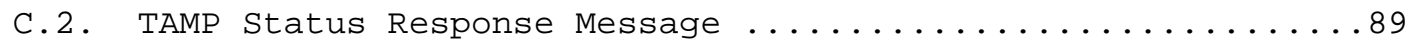

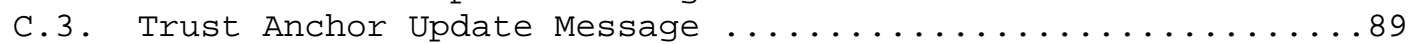

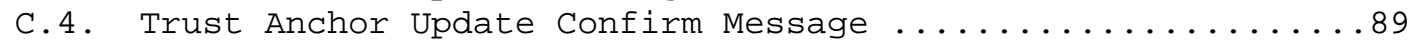

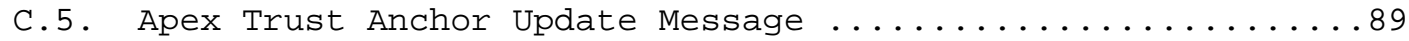

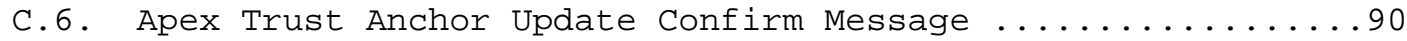

C.7. Community Update Message .......................990

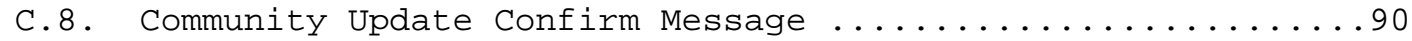

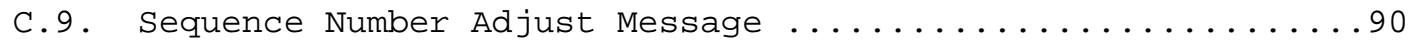

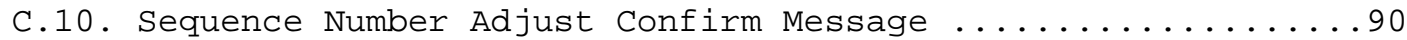

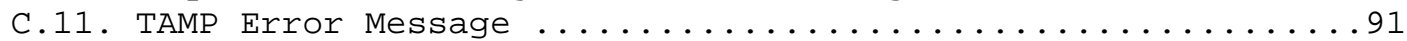

1. Introduction

This document describes the Trust Anchor Management Protocol (TAMP). TAMP may be used to manage the trust anchors and community identifiers in any device that uses digital signatures; however, this specification was written with the requirements of cryptographic modules in mind. For example, TAMP can support signed firmware packages [RFC4108], where the trust anchor public key can be used to validate digital signatures on firmware packages or validate the X.509 certification path [RFC5280][X.509] of the firmware package signer.

Most TAMP messages are digitally signed to provide integrity protection and data origin authentication. Both signed and unsigned TAMP messages employ the Cryptographic Message Syntax (CMS)

[RFC5652]. The CMS is a data protection encapsulation syntax that makes use of ASN.1 [X.680]. 
This specification does not provide for confidentiality of TAMP messages. If confidentiality is required, then the communications environment that is used to transfer TAMP messages must provide it. This specification is intended to satisfy the protocol-related requirements expressed in "Trust Anchor Management Requirements" [TA-MGMT-REQS] and uses vocabulary from that document.

TAMP messages may be exchanged in real time over a network, such as via HTTP as described in Appendix A, or may be stored and transferred using other means. TAMP exchanges consist of a request message that includes instructions for a trust anchor store and, optionally, a corresponding response message that reports the result of carrying out the instructions in the request. Response messages need not be propagated in all cases. For example, a GPS receiver may be unable to transmit a response and may instead use an attached display to indicate the results of processing a TAMP request.

\subsection{Terminology}

The key words "MUST", "MUST NOT", "REQUIRED", "SHALL", "SHALL NOT", "SHOULD", "SHOULD NOT", "RECOMMENDED", "MAY", and "OPTIONAL" in this document are to be interpreted as described in RFC 2119 [RFC2119].

\subsection{Trust Anchors}

TAMP manages trust anchors. A trust anchor contains a public key that is used to validate digital signatures. TAMP recognizes three formats for representing trust anchor information: Certificate [RFC5280], TBSCertificate [RFC5280], and TrustAnchorInfo [RFC5914].

All trust anchors are distinguished by the public key, and all trust anchors consist of the following components:

- A public key signature algorithm identifier and associated public key, which MAY include parameters

- A public key identifier

Other information may appear in a trust anchor, including certification path processing controls and a human readable name.

TAMP recognizes three types of trust anchors based on functionality: apex trust anchors, management trust anchors, and identity trust anchors.

In addition to the information described above, apex trust anchors and management trust anchors that sign TAMP messages have an associated sequence number that is used for replay detection. 
The public key is used to name a trust anchor, and the public key identifier is used to identify the trust anchor as a signer of a particular object, such as a SignedData object or a public key certificate. This public key identifier can be stored with the trust anchor, or in most public key identifier assignment methods, it can be computed from the public key whenever needed.

A trust anchor public key can be used in two different ways to support digital signature validation. In the first approach, the trust anchor public key is used directly to validate the digital signature. In the second approach, the trust anchor public key is used to validate an X.509 certification path, and then the subject public key in the final certificate in the certification path is used to validate the digital signature. When the second approach is employed, the certified public key may be used for things other than digital signature validation; the other possible actions are constrained by the key usage certificate extension.

TAMP implementations MUST support validation of TAMP messages that are directly validated using a trust anchor. Support for TAMP messages validated using an X.509 certificate validated using a trust anchor, or using longer certification paths, is OPTIONAL. The CMS provides a location to carry X.509 certificates, and this facility can be used to transfer certificates to aid in the construction of the certification path.

\subsubsection{Apex Trust Anchors}

Within the context of a single trust anchor store, one trust anchor is superior to all others. This trust anchor is referred to as the apex trust anchor. This trust anchor represents the ultimate authority over the trust anchor store. Much of this authority can be delegated to other trust anchors.

The apex trust anchor private key is expected to be controlled by an entity with information assurance responsibility for the trust anchor store. The apex trust anchor is by definition unconstrained and therefore does not have explicit authorization information associated with it.

Due to the special nature of the apex trust anchor, TAMP includes separate facilities to change it. In particular, TAMP includes a facility to securely replace the apex trust anchor. This action might be taken for one or more of the following reasons:

- The crypto period for the apex trust anchor public/private key pair has come to an end 
- The apex trust anchor private key is no longer available

- The apex trust anchor public/private key pair needs to be revoked

- The authority has decided to use a different digital signature algorithm or the same digital signature algorithm with different parameters, such as a different elliptic curve

- The authority has decided to use a different key size

- The authority has decided to transfer control to another authority

To accommodate these requirements, the apex trust anchor MAY include two public keys. Whenever the apex trust anchor is updated, both public keys will be replaced. The first public key, called the operational public key, is used in the same manner as other trust anchors. Any type of TAMP message, including an Apex Trust Anchor Update message, can be validated with the operational public key. The second public key, called the contingency public key, can only be used to update the apex trust anchor. The contingency private key SHOULD be used at only one point in time; it is used only to sign an Apex Trust Anchor Update message that results in its own replacement (as well as the replacement of the operational public key). The contingency public key is distributed in encrypted form. When the contingency public key is used to validate an Apex Trust Anchor Update message, the symmetric key needed to decrypt the contingency public key is provided as part of the signed Apex Trust Anchor Update message that is to be verified with the contingency public key.

\subsubsection{Management Trust Anchors}

Management trust anchors are used in the management of cryptographic modules. For example, the TAMP messages specified in this document are validated to a management trust anchor. Likewise, a signed firmware package as specified in [RFC4108] is validated to a management trust anchor.

\subsubsection{Identity Trust Anchors}

Identity trust anchors are used to validate certification paths, and they represent the trust anchor for a public key infrastructure. They are most often used in the validation of certificates associated with non-management applications. 


\subsection{Architectural Elements}

TAMP does not assume any particular architecture. However, TAMP REQUIRES the following architectural elements: a cryptographic module, a trust anchor store, TAMP protocol processing, and other application-specific protocol processing.

A globally unique algorithm identifier MUST be assigned for each oneway hash function, digital signature generation/validation algorithm, and symmetric key unwrapping algorithm that is implemented. To support CMS, an object identifier (OID) is assigned to name a one-way hash function, and another OID is assigned to name each combination of a one-way hash function when used with a digital signature algorithm. Similarly, certificates associate OIDs assigned to public key algorithms with subject public keys, and certificates make use of an OID that names both the one-way hash function and the digital signature algorithm for the certificate issuer digital signature. [RFC3279], [RFC3370], [RFC5753], and [RFC5754] provide OIDs for a number of commonly used algorithms; however, OIDs may be defined in later or different specifications.

\subsubsection{Cryptographic Module}

The cryptographic module MUST include the following capabilities:

- The cryptographic module SHOULD support the secure storage of a digital signature private key to sign TAMP responses and either a certificate containing the associated public key or a certificate designator. In the latter case, the certificate is stored elsewhere but is available to parties that need to validate cryptographic module digital signatures. The designator is a public key identifier.

- The cryptographic module MUST support at least one one-way hash function, one digital signature validation algorithm, one digital signature generation algorithm, and, if contingency keys are supported, one symmetric key unwrapping algorithm. If only one one-way hash function is present, it MUST be consistent with the digital signature validation and digital signature generation algorithms. If only one digital signature validation algorithm is present, it MUST be consistent with the apex trust anchor operational public key. If only one digital signature generation algorithm is present, it MUST be consistent with the cryptographic module digital signature private key. These algorithms MUST be available for processing TAMP messages, including the content types defined in [RFC5652], and for validation of X.509 
certification paths. As with similar specifications, such as RFC 5280, this specification does not mandate support for any cryptographic algorithms. However, algorithm requirements may be imposed by specifications that use trust anchors managed via TAMP.

\subsubsection{Trust Anchor Store}

The trust anchor store MUST include the following capabilities:

- Each trust anchor store MUST have a unique name. For example, a cryptographic module containing a single trust anchor store may be identified by a unique serial number with respect to other modules within the same family where the family is represented as an ASN.1 object identifier (OID) and the unique serial number is represented as a string of octets. Other means of establishing a unique name are also possible.

- Each trust anchor store SHOULD have the capability to securely store one or more community identifiers. The community identifier is an OID, and it identifies a collection of cryptographic modules that can be the target of a single TAMP message or the intended recipients for a particular management message.

- The trust anchor store SHOULD support the use of an apex trust anchor. If apex support is provided, the trust anchor store MUST support the secure storage of exactly one apex trust anchor. The trust anchor store SHOULD support the secure storage of at least one additional trust anchor. Each trust anchor MUST contain a unique public key. A public key MUST NOT appear more than once in a trust anchor store.

- The trust anchor store MUST have the capability to securely store a sequence number for each trust anchor authorized to generate TAMP messages and be able to report the sequence number along with the key identifier of the trust anchor.

\subsubsection{TAMP Processing Dependencies}

TAMP processing MUST include the following capabilities:

- TAMP processing MUST have a means of locating an appropriate trust anchor. Two mechanisms are available. The first mechanism is based on the public key identifier for digital signature verification, and the second mechanism is based on the trust anchor X.500 distinguished name and other X.509 certification path controls for certificate path discovery and validation. The first mechanism MUST be supported, but the second mechanism MAY be supported. 
- TAMP processing MUST be able to invoke the digital signature validation algorithm using the public key held in secure storage for trust anchors.

- TAMP processing MUST have read and write access to secure storage for sequence numbers associated with each TAMP message signer as described in section 6.

- TAMP processing MUST have read and write access to secure storage for trust anchors in order to update them. Update operations include adding trust anchors, removing trust anchors, and modifying trust anchors. Application-specific constraints MUST be securely stored with each management trust anchor as described in Section 1.3.4.

- TAMP processing MUST have read access to secure storage for the community membership list, if any, to determine whether a targeted message ought to be accepted.

- To implement the OPTIONAL community identifier update feature, TAMP processing MUST have read and write access to secure storage for the community membership list.

- To generate signed confirmation messages, TAMP processing MUST be able to invoke the digital signature generation algorithm using the cryptographic module digital signature private key, and it MUST have read access to the cryptographic module certificate or its designator. TAMP uses X.509 certificates [RFC5280].

- The TAMP processing MUST have read access to the trust anchor store unique name.

\subsubsection{Application-Specific Protocol Processing}

The apex trust anchor and management trust anchors managed with TAMP can be used by the TAMP application. Other management applications MAY make use of all three types of trust anchors, but non-management applications SHOULD only make use of identity trust anchors. Applications MUST ensure that usage of a trust anchor is consistent with any constraints associated with the trust anchor. For example, if name constraints are associated with a trust anchor, certification paths that start with the trust anchor and contain certificates with names that violate the name constraints MUST be rejected.

The application-specific protocol processing MUST be provided with the following services: 
- The application-specific protocol processing MUST have a means of locating an appropriate trust anchor. Two mechanisms are available to applications. The first mechanism is based on the public key identifier for digital signature verification, and the second mechanism is based on the trust anchor X.500 distinguished name and other X.509 certification path controls for certificate path discovery and validation.

- The application-specific protocol processing MUST be able to invoke the digital signature validation algorithm using the public key held in secure storage for trust anchors.

- The application-specific protocol processing MUST have read access to data associated with trust anchors to ensure that constraints can be enforced appropriately. For example, an application MUST have read access to any name constraints associated with a TA to ensure that certification paths terminated by that TA do not include certificates issued to entities outside the TA managerdesignated namespace.

- The application-specific protocol processing MUST have read access to secure storage for the community membership list, if any, to determine whether a targeted message ought to be accepted.

- If the application-specific protocol requires digital signatures on confirmation messages or receipts, then the applicationspecific protocol processing MUST be able to invoke the digital signature generation algorithm with the cryptographic module digital signature private key and its associated certificate or certificate designator. Digital signature generation MUST be controlled in a manner that ensures that the content type of signed confirmation messages or receipts is appropriate for the application-specific protocol processing.

- The application-specific protocol processing MUST have read access to the trust anchor store unique name.

\subsection{ASN.1 Encoding}

The CMS uses Abstract Syntax Notation One (ASN.1) [X.680]. ASN.1 is a formal notation used for describing data protocols, regardless of the programming language used by the implementation. Encoding rules describe how the values defined in ASN.1 will be represented for transmission. The Basic Encoding Rules (BER) [X.690] are the most widely employed rule set, but they offer more than one way to represent data structures. For example, definite-length encoding and indefinite-length encoding are supported. This flexibility is not desirable when digital signatures are used. As a result, the 
Distinguished Encoding Rules (DER) [X.690] were invented. DER is a subset of BER that ensures a single way to represent a given value. For example, DER always employs definite-length encoding.

Digitally signed structures MUST be encoded with DER. In other specifications, structures that are not digitally signed do not require DER, but in this specification, DER is REQUIRED for all structures. By always using DER, the TAMP processor will have fewer options to implement.

ASN.1 is used throughout the text of this document for illustrative purposes. The authoritative source of ASN.1 for the structures defined in this document is Appendix A.

\section{Cryptographic Message Syntax Profile}

TAMP makes use of signed and unsigned messages. The Cryptographic Message Syntax (CMS) is used in both cases. A digital signature is used to protect the message from undetected modification and provide data origin authentication. TAMP makes no general provision for encryption of content.

CMS is used to construct a signed TAMP message. The CMS Contentinfo content type MUST always be present. For signed messages, ContentInfo MUST encapsulate the CMS SignedData content type; for unsigned messages, ContentInfo MUST encapsulate the TAMP message directly. The CMS SignedData content type MUST encapsulate the TAMP message. A unique content type identifier identifies the particular type of TAMP message. The CMS encapsulation of a signed TAMP message is summarized by:

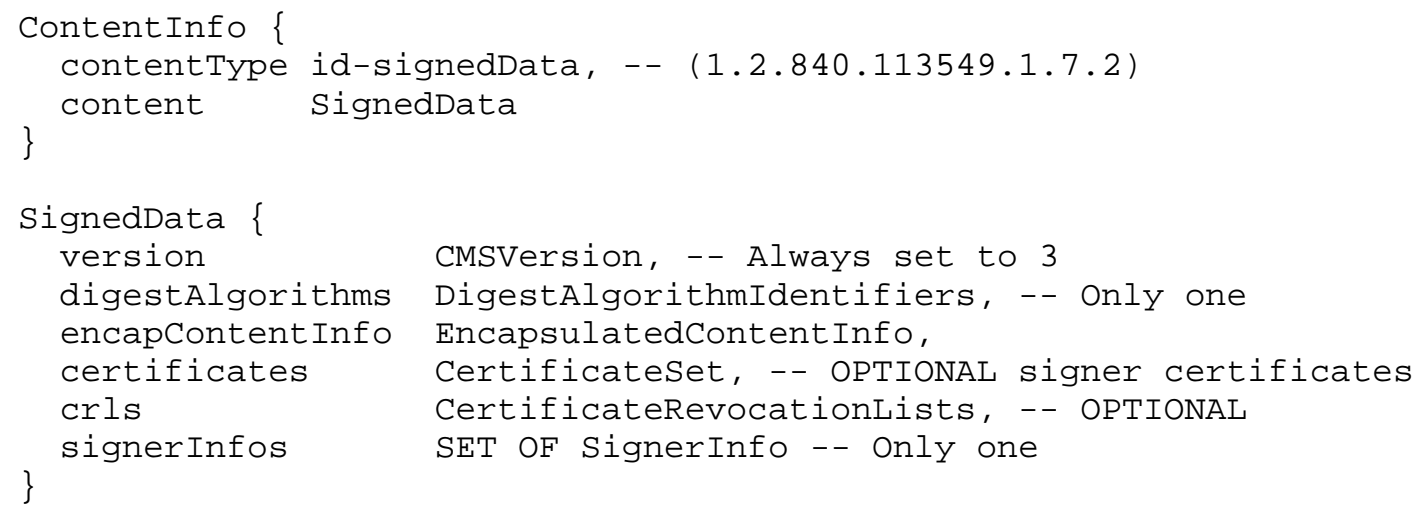




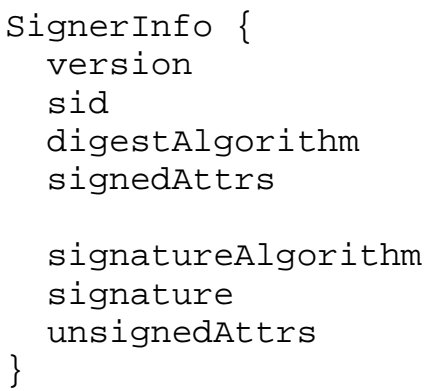

When a TAMP message is used to update the apex trust anchor, this same structure is used; however, the digital signature will be validated with either the apex trust anchor operational public key or the contingency public key. When the contingency public key is used, the symmetric key needed to decrypt the previously stored contingency public key is provided as a contingency-public-key-decrypt-key unsigned attribute. Section 4.5 of this document describes the Apex Trust Anchor Update message.

CMS is also used to construct an unsigned TAMP message. The CMS ContentInfo structure MUST always be present, and it MUST be the outermost layer of encapsulation. A unique content type identifier identifies the particular TAMP message. The CMS encapsulation of an unsigned TAMP message is summarized by:

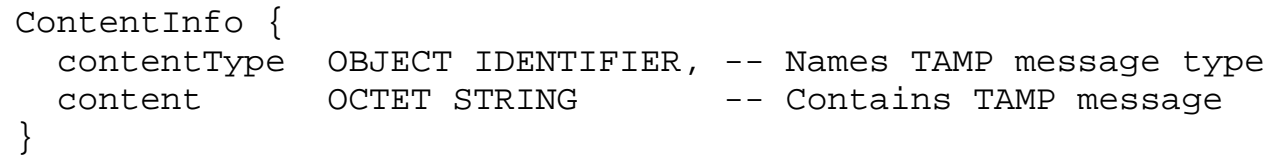

\subsection{Contentinfo}

CMS requires the outermost encapsulation to be ContentInfo [RFC5652].

The fields of ContentInfo are used as follows:

- contentType indicates the type of the associated content, and for TAMP, the encapsulated type is either signedData or the content type identifier associated with an unsigned TAMP message. When the id-signedData (1.2.840.113549.1.7.2) object identifier is present in this field, then a signed TAMP message is in the content. Otherwise, an unsigned TAMP message is in the content. 
- content holds the content, and for TAMP, the content is either a SignedData content or an unsigned TAMP message.

\subsection{SignedData Info}

The SignedData content type [RFC5652] contains the signed TAMP message and a digital signature value; the signedData content type MAY also contain the certificates needed to validate the digital signature. The fields of SignedData are used as follows:

- version is the syntax version number, and for TAMP, the version number MUST be set to 3 .

- digestAlgorithms is a collection of one-way hash function identifiers, and for TAMP, it contains a single one-way hash function identifier. The one-way hash function employed by the TAMP message originator in generating the digital signature MUST be present.

- encapcontentInfo is the signed content, consisting of a content type identifier and the content itself. The use of the EncapsulatedContentInfo type is discussed further in Section 2.2.2.

- certificates is an OPTIONAL collection of certificates. It MAY be omitted, or it MAY include the X.509 certificates needed to construct the certification path of the TAMP message originator. For TAMP messages sent to a trust anchor store where an apex trust anchor or management trust anchor is used directly to validate the TAMP message digital signature, this field SHOULD be omitted. When an apex trust anchor or management trust anchor is used to validate an X.509 certification path [RFC5280], and the subject public key from the final certificate in the certification path is used to validate the TAMP message digital signature, the certificate of the TAMP message originator SHOULD be included, and additional certificates to support certification path construction MAY be included. For TAMP messages sent by a trust anchor store, this field SHOULD include only the signer's certificate or should be omitted. A TAMP message recipient MUST NOT reject a valid TAMP message that contains certificates that are not needed to validate the digital signature. PKCS\#6 extended certificates [PKCS\#6] and attribute certificates (either version 1 or version 2) [RFC5755] MUST NOT be included in the set of certificates; these certificate formats are not used in TAMP. Certification authority (CA) certificates and end entity certificates MUST conform to the profiles defined in [RFC5280]. 
- crls is an OPTIONAL collection of certificate revocation lists (CRLS).

- signerInfos is a collection of per-signer information, and for TAMP, the collection MUST contain exactly one SignerInfo. The use of the SignerInfo type is discussed further in section 2.2.1.

\subsubsection{SignerInfo}

The TAMP message originator is represented in the SignerInfo type.

The fields of SignerInfo are used as follows:

- version is the syntax version number. With TAMP, the version MUST be set to 3 .

- sid identifies the TAMP message originator's public key. The subjectKeyIdentifier alternative is always used with TAMP, which identifies the public key directly. When the public key is included in a TrustAnchorInfo object, this identifier is included in the keyId field. When the public key is included in a Certificate or TBSCertificate, this identifier is included in the subjectKeyIdentifier certificate extension.

- digestAlgorithm identifies the one-way hash function, and any associated parameters, used by the TAMP message originator. It MUST contain the one-way hash functions employed by the originator. This message digest algorithm identifier MUST match the one carried in the digestAlgorithms field in SignedData. The message digest algorithm identifier is carried in two places to facilitate stream processing by the receiver.

- signedAttrs is an OPTIONAL set of attributes that are signed along with the content. The signedAttrs are OPTIONAL in the CMS, but signedAttrs is REQUIRED for all signed TAMP messages. The SET OF Attribute MUST be encoded with the Distinguished Encoding Rules (DER) [X.690]. Section 2.2.3 of this document lists the signed attributes that MUST be included in the collection. Other signed attributes MAY be included, but any unrecognized signed attributes MUST be ignored.

- signatureAlgorithm identifies the digital signature algorithm, and any associated parameters, used by the TAMP message originator to generate the digital signature.

- signature is the digital signature value generated by the TAMP message originator. 
- unsignedAttrs is an OPTIONAL set of attributes that are not signed. For TAMP, this field is usually omitted. It is present only in Apex Trust Anchor Update messages that are to be validated using the apex trust anchor contingency public key. In this case, the SET OF Attribute MUST include the symmetric key needed to decrypt the contingency public key in the contingency-public-keydecrypt-key unsigned attribute. Section 2.2.4 of this document describes this unsigned attribute.

\subsubsection{EncapsulatedContentInfo}

The EncapsulatedContentInfo structure contains the TAMP message. The fields of EncapsulatedContentInfo are used as follows:

- econtentType is an object identifier that uniquely specifies the content type, and for TAMP, the value identifies the TAMP message. The list of TAMP message content types is provided in Section 4.

o eContent is the TAMP message, encoded as an octet string. In general, the CMS does not require the eContent to be DER-encoded before constructing the octet string. However, TAMP messages MUST be DER-encoded.

\subsubsection{Signed Attributes}

The TAMP message originator MUST digitally sign a collection of attributes along with the TAMP message. Each attribute in the collection MUST be DER-encoded. The syntax for attributes is defined in [RFC5912].

Each of the attributes used with this CMS profile has a single attribute value. Even though the syntax is defined as a SET OF AttributeValue, there MUST be exactly one instance of AttributeValue present.

The SignedAttributes syntax within SignerInfo is defined as a SET OF Attribute. The SignedAttributes MUST include only one instance of any particular attribute. TAMP messages that violate this rule MUST be rejected as malformed.

The TAMP message originator MUST include the content-type and message-digest attributes. The TAMP message originator MAY also include the binary-signing-time attribute. 
The TAMP message originator MAY include any other attribute that it deems appropriate. The intent is to allow additional signed attributes to be included if a future need is identified. This does not cause an interoperability concern because unrecognized signed attributes MUST be ignored.

The following summarizes the signed attribute requirements for TAMP messages:

- content-type MUST be supported.

- message-digest MUST be supported.

o binary-signing-time MAY be supported. When present, it is generally ignored by the recipient.

- other attributes MAY be supported. Unrecognized attributes MUST be ignored by the recipient.

\subsubsection{Content-Type Attribute}

The TAMP message originator MUST include a content-type attribute; it is an object identifier that uniquely specifies the content type. Section 11.1 of [RFC5652] defines the content-type attribute. For TAMP, the value identifies the TAMP message. The list of TAMP message content types and their identifiers is provided in Section 4.

A content-type attribute MUST contain the same object identifier as the content type contained in the EncapsulatedContentInfo.

\subsubsection{Message-Digest Attribute}

The TAMP message originator MUST include a message-digest attribute, having as its value the output of a one-way hash function computed on the TAMP message that is being signed. Section 11.2 of [RFC5652] defines the message-digest attribute.

\subsubsection{Binary-Signing-Time Attribute}

The TAMP message originator MAY include a binary-signing-time attribute, specifying the time at which the digital signature was applied to the TAMP message. The binary-signing-time attribute is defined in [RFC4049].

No processing of the binary-signing-time attribute is REQUIRED of a TAMP message recipient; however, the binary-signing-time attribute MAY be included by the TAMP message originator as a form of message identifier. 


\subsubsection{Unsigned Attributes}

For TAMP, unsigned attributes are usually omitted. An unsigned attribute is present only in Apex Trust Anchor Update messages that are to be validated by the apex trust anchor contingency public key. In this case, the symmetric key to decrypt the previous contingency public key is provided in the contingency-public-key-decrypt-key unsigned attribute. This attribute MUST be supported, and it is described in section 2.2.4.1.

The TAMP message originator SHOULD NOT include other unsigned attributes, and any unrecognized unsigned attributes MUST be ignored.

The UnsignedAttributes syntax within SignerInfo is defined as a SET OF Attribute. The UnsignedAttributes MUST include only one instance of any particular attribute. TAMP messages that violate this rule MUST be rejected as malformed.

2.2.4.1. Contingency-Public-Key-Decrypt-Key Attribute

The contingency-public-key-decrypt-key attribute provides the plaintext symmetric key needed to decrypt the previously distributed apex trust anchor contingency public key. The symmetric key MUST be useable with the symmetric algorithm used to previously encrypt the contingency public key.

The contingency-public-key-decrypt-key attribute has the following syntax:

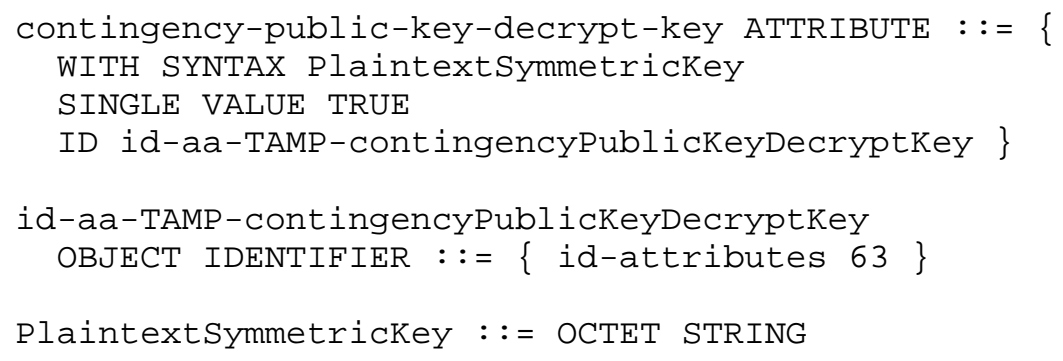

3. Trust Anchor Formats

TAMP recognizes three formats for representing trust anchor information within the protocol itself: Certificate [RFC5280], TBSCertificate [RFC5280], and TrustAnchorInfo [RFC5914]. The TrustAnchorChoice structure, defined in [RFC5914], is used to select one of these options. 


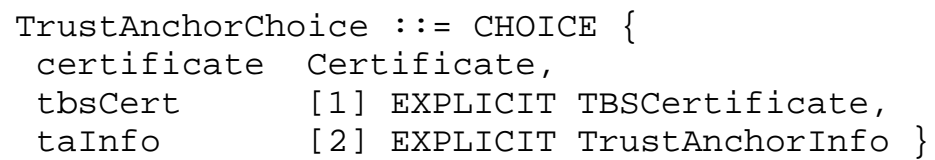

The Certificate structure is commonly used to represent trust anchors. Certificates include a signature, which removes the ability for relying parties to customize the information within the structure itself. TBSCertificate contains all of the information of the Certificate structure except for the signature, enabling tailoring of the information. TrustAnchorInfo is intended to serve as a minimalist representation of trust anchor information for scenarios where storage or bandwidth is highly constrained.

Implementations are not required to support all three options. The unsupportedTrustAnchorFormat error code should be indicated when generating a TAMPError due to receipt of an unsupported trust anchor format.

4. Trust Anchor Management Protocol Messages

TAMP makes use of signed and unsigned messages. The CMS is used in both cases. An object identifier is assigned to each TAMP message type, and this object identifier is used as a content type in the CMS .

TAMP specifies eleven message types. The following provides the content type identifier for each TAMP message type, and it indicates whether a digital signature is required. If the following indicates that the TAMP message MUST be signed, then implementations MUST reject a message of that type that is not signed.

- The TAMP Status Query message MUST be signed. It uses the following object identifier: \{id-tamp 1 .

- The TAMP Status Response message SHOULD be signed. It uses the following object identifier: \{id-tamp 2 \}.

- The Trust Anchor Update message MUST be signed. It uses the following object identifier: $\{$ id-tamp 3$\}$.

- The Trust Anchor Update Confirm message SHould be signed. It uses the following object identifier: $\{$ id-tamp 4 \}.

- The Apex Trust Anchor Update message MUST be signed. It uses the following object identifier: \{id-tamp 5 . 
- The Apex Trust Anchor Update Confirm message SHOULD be signed. It uses the following object identifier: \{id-tamp 6 .

- The Community Update message MUST be signed. It uses the following object identifier: \{id-tamp 7 .

- The Community Update Confirm message SHould be signed. It uses the following object identifier: $\{$ id-tamp 8$\}$.

- The Sequence Number Adjust MUST be signed. It uses the following object identifier: $\{$ id-tamp 10$\}$.

- The Sequence Number Adjust Confirm message SHOULD be signed. It uses the following object identifier: \{id-tamp 11$\}$.

- The TAMP Error message SHOULD be signed. It uses the following object identifier: \{id-tamp 9 .

Trust anchor managers generate TAMP Status Query, Trust Anchor Update, Apex Trust Anchor Update, Community Update, and Sequence Number Adjust messages. Trust anchor stores generate TAMP status Response, Trust Anchor Update Confirm, Apex Trust Anchor Update Confirm, Community Update Confirm, Sequence Number Adjust Confirm, and TAMP Error messages.

Support for Trust Anchor Update messages is REQUIRED. Support for all other message formats is RECOMMENDED. Implementations that support the HTTP binding described in Appendix C MUST additionally support Trust Anchor Update Confirm and TAMP Error messages and MAY support 0 or more of the following pairs of messages: TAMP Status Query and TAMP Status Query Response; Apex Trust Anchor Update and Apex Trust Anchor Update Confirm; Community Update and Community Update Confirm; Sequence Number Adjust and Sequence Number Adjust Confirm. Implementations that operate in a disconnected manner MUST NOT assume a response will be received from each consumer of a TAMP message.

A typical interaction between a trust anchor manager and a trust anchor store will follow the message flow shown in Figure 1 . Figure 1 does not illustrate a flow where an error occurs. 


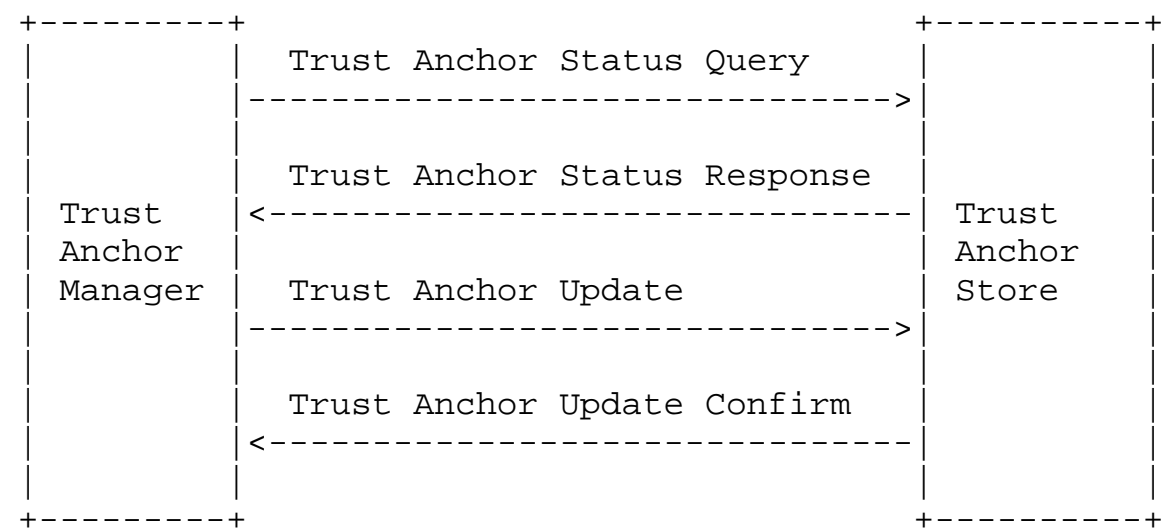

Figure 1. Typical TAMP Message Flow

Each TAMP query and update message includes an indication of the type of response that is desired. The response can either be terse or verbose. All trust anchor stores MUST support both the terse and verbose responses and SHOULD generate a response of the type indicated in the corresponding request. TAMP response processors MUST support processing of both terse and verbose responses.

Trust anchor stores SHOULD be able to process and properly act upon the valid payload of the TAMP Status Query message, the Trust Anchor Update message, the Apex Trust Anchor Update message, and the Sequence Number Adjust message. TAMP implementations MAY also process and act upon the valid payload of the Community Update message.

TAMP implementations SHOULD support generation of the TAMP Status Response message, the Trust Anchor Update Confirm message, the Apex Trust Anchor Update Confirm message, the Sequence Number Adjust Confirm message, and the TAMP Error message. If a TAMP implementation supports the Community Update message, then generation of Community Update Confirm messages SHOULD also be supported.

\subsection{TAMP Status Query}

The TAMP Status Query message is used to request information about the trust anchors that are currently installed in a trust anchor store, and for the list of communities to which the store belongs. The TAMP Status Query message MUST be signed. For the query message to be valid, the trust anchor store MUST be an intended recipient of the query; the sequence number checking described in section 6 MUST be successful when the TAMP message signer is a trust anchor; and the digital signature MUST be validated by the apex trust anchor 


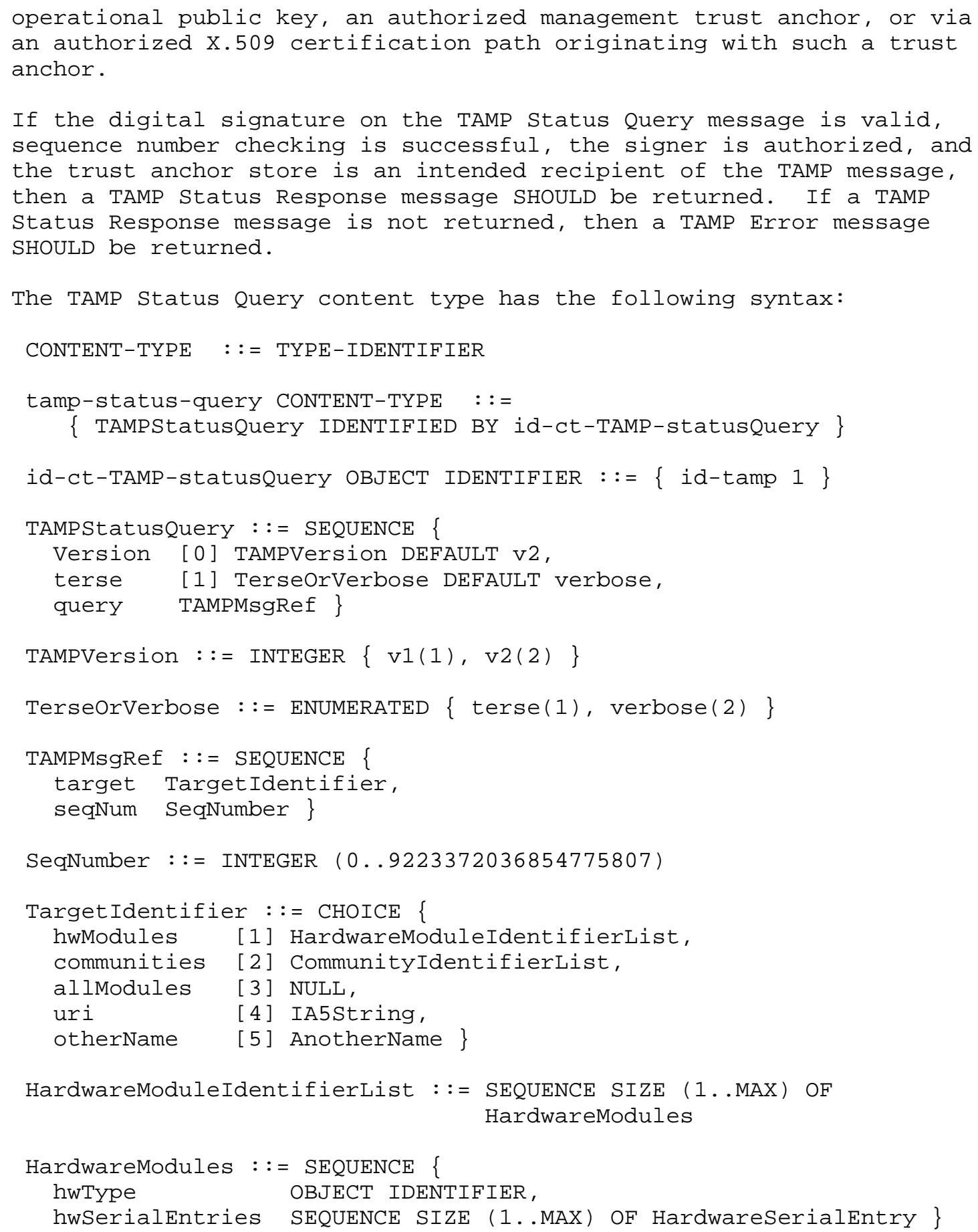




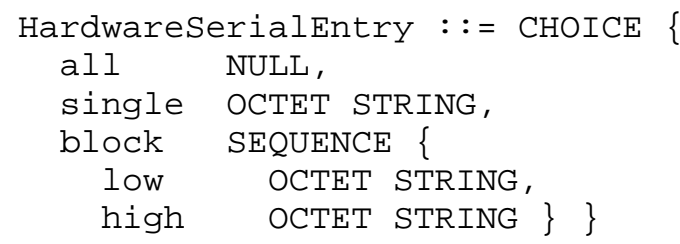

CommunityIdentifierList : := SEQUENCE SIZE (0..MAX) OF Community

Community $::=$ OBJECT IDENTIFIER

The fields of TAMPStatusQuery are used as follows:

- version identifies version of TAMP. For this version of the specification, the default value, v2, MUST be used.

- terse indicates the type of response that is desired. A terse response is indicated by a value of 1 , and a verbose response is indicated by a value of 2 , which is omitted during encoding since it is the default value.

- query contains two items: the target and the seqNum. target identifies the target(s) of the query message. seqNum is a single-use value that will be used to match the TAMP Status Query message with the TAMP Status Response message. The sequence number is also used to detect TAMP message replay. The sequence number processing described in section 6 MUST successfully complete before a response is returned.

The fields of TAMPMsgRef are used as follows:

- target identifies the target(s) of the query. Several alternatives for naming a target are provided. To identify a cryptographic module, a combination of a cryptographic type and serial number are used. The cryptographic type is represented as an ASN.1 object identifier, and the unique serial number is represented as a string of octets. To facilitate compact representation of serial numbers, a contiguous block can be specified by the lowest included serial number and the highest included serial number. When present, the high and low octet strings MUST have the same length. The HardwareModuleIdentifierList sequence MUST NOT contain duplicate hwType values, so that each member of the sequence names all of the cryptographic modules of this type. Object identifiers are also used to identify communities of trust anchor stores. A sequence of these object identifiers is used if more than one community is the target of the message. A trust anchor store is considered a target if it is a member of any of the listed 
communities. An explicit NULL value is used to identify all modules that consider the signer of the TAMP message to be an authorized source for that message type. The uri field can be used to identify a target, i.e., a trust anchor store, using a Uniform Resource Identifier [RFC3986]. Additional name types are supported via the otherName field, which is of type AnotherName. AnotherName is defined in [RFC5280]. The format and semantics of the name are indicated through the OBJECT IDENTIFIER in the typeid field. The name itself is conveyed as a value field in otherName. Implementations MUST support the allModules option and SHOULD support all TargetIdentifier options.

- seqNum contains a single-use value that will be used to match the TAMP Status Query message with the successful TAMP Status Response message. The sequence number processing described in section 6 MUST successfully complete before a response is returned.

To determine whether a particular cryptographic module serial number is considered part of a specified block, all of the following conditions MUST be met. First, the cryptographic module serial number MUST be the same length as both the high and low octet strings. Second, the cryptographic module serial number MUST be greater than or equal to the low octet string. Third, the cryptographic module serial number MUST be less than or equal to the high octet string.

One octet string is equal to another if they are of the same length and are the same at each octet position. An octet string, S1, is greater than another, S2, where S1 and S2 have the same length, if and only if S1 and S2 have different octets in one or more positions, and in the first such position, the octet in $\mathrm{S} 1$ is greater than that in S2, considering the octets as unsigned binary numbers. Note that these octet string comparison definitions are consistent with those in clause 6 of [X.690].

\subsection{TAMP Status Query Response}

The TAMP Status Response message is a reply by a trust anchor store to a valid TAMP Status Query message. The TAMP Status Response message provides information about the trust anchors that are currently installed in the trust anchor store and the list of communities to which the trust anchor store belongs, if any. The TAMP Status Response message MAY be signed or unsigned. A TAMP Status Response message MUST be signed if the implementation is capable of signing it. 
The TAMP Status Response content type has the following syntax:

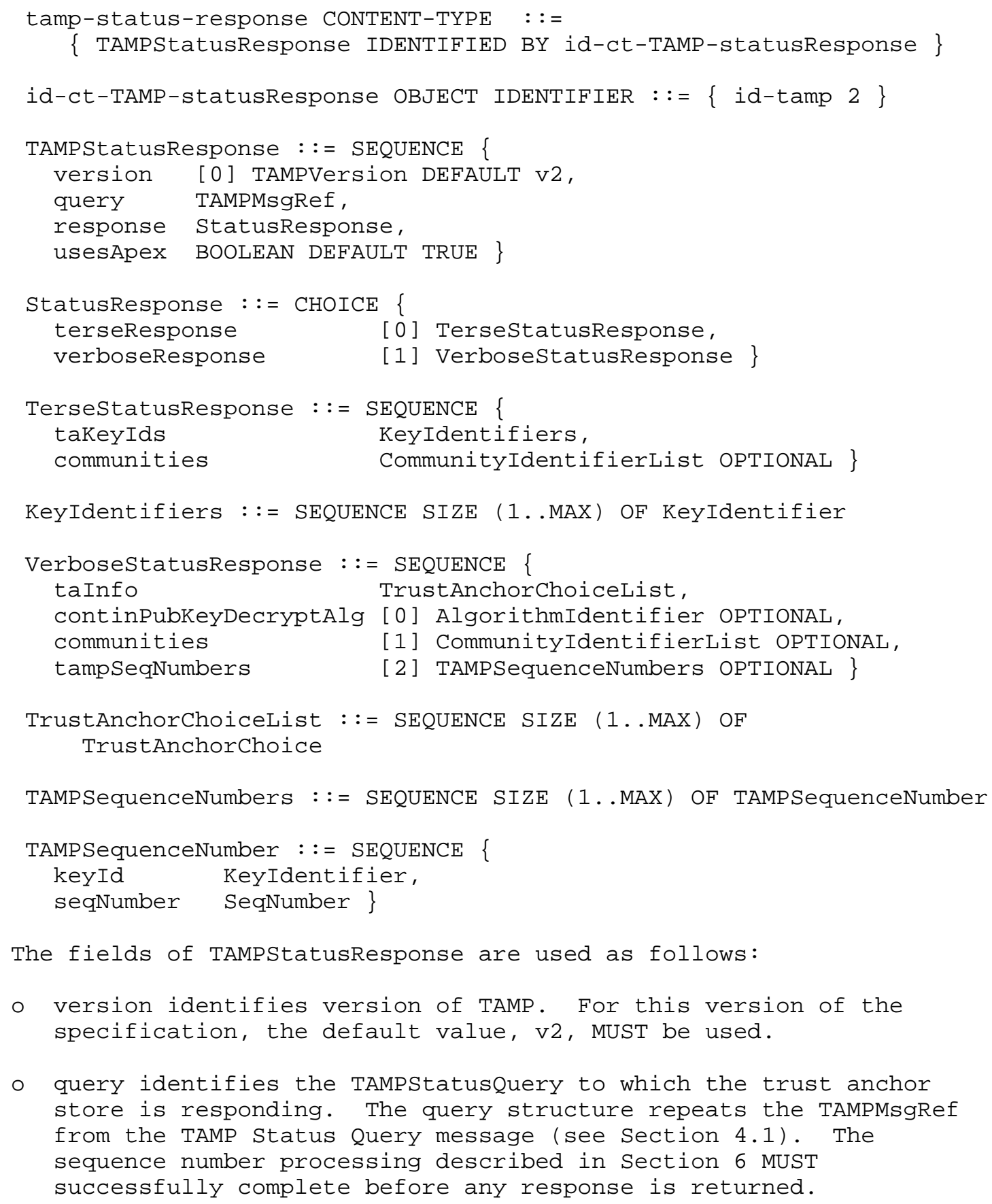


- response contains either a terse response or a verbose response. The terse response is represented by TersestatusResponse, and the verbose response is represented by VerbosestatusResponse.

- usesApex is a Boolean value that indicates whether the first item in the TersestatusResponse.takeyIds or VerboseStatusResponse.taInfo field identifies the apex TA.

The fields of TersestatusResponse are used as follows:

- takeyIds contains a sequence of key identifiers. Each trust anchor contained in the trust anchor store is represented by one key identifier. When TAMPStatusResponse.usesApex is TRUE, the apex trust anchor is represented by the first key identifier in the sequence, which contains the key identifier of the operational public key.

- communities is OPTIONAL. When present, it contains a sequence of object identifiers. Each object identifier names one community to which this trust anchor store belongs. When the trust anchor store belongs to no communities, this field is omitted.

The fields of VerbosestatusResponse are used as follows:

- taInfo contains a sequence of TrustAnchorchoice structures. One entry in the sequence is provided for each trust anchor contained in the trust anchor store. When TAMPStatusResponse.usesApex is TRUE, the apex trust anchor is the first trust anchor in the sequence.

o continPubKeyDecryptAlg is OPTIONAL. When present, it indicates the decryption algorithm needed to decrypt the currently installed apex trust anchor contingency public key, if a contingency key is associated with the apex trust anchor. When present, TAMPStatusResponse.usesApex MUST be TRUE.

- communities is OPTIONAL. When present, it contains a sequence of object identifiers. Each object identifier names one community to which this trust anchor store belongs. When the trust anchor store belongs to no communities, this field is omitted.

- tampseqNumbers is OPTIONAL. When present, it is used to indicate the currently held sequence number for each trust anchor authorized to sign TAMP messages. The keyId field identifies the trust anchor, and the seqNumber field provides the current sequence number associated with the trust anchor. 


\subsection{Trust Anchor Update}

The Trust Anchor Update message is used to add, remove, and change management and identity trust anchors. The Trust Anchor Update message cannot be used to update the apex trust anchor. The Trust Anchor Update message MUST be signed. For a Trust Anchor Update message to be valid, the trust anchor store MUST be an intended recipient of the update; the sequence number checking described in Section 6 MUST be successful when the TAMP message signer is a trust anchor; and the digital signature MUST be validated using the apex trust anchor operational public key, an authorized management trust anchor, or via an authorized X.509 certification path originating with such a trust anchor.

If the digital signature on the Trust Anchor Update message is valid, sequence number checking is successful, the signer is authorized, and the trust anchor store is an intended recipient of the TAMP message, then the trust anchor store MUST perform the specified updates and return a Trust Anchor Update Confirm message. If a Trust Anchor Update Confirm message is not returned, then a TAMP Error message SHOULD be returned.

The Trust Anchor Update content type has the following syntax:

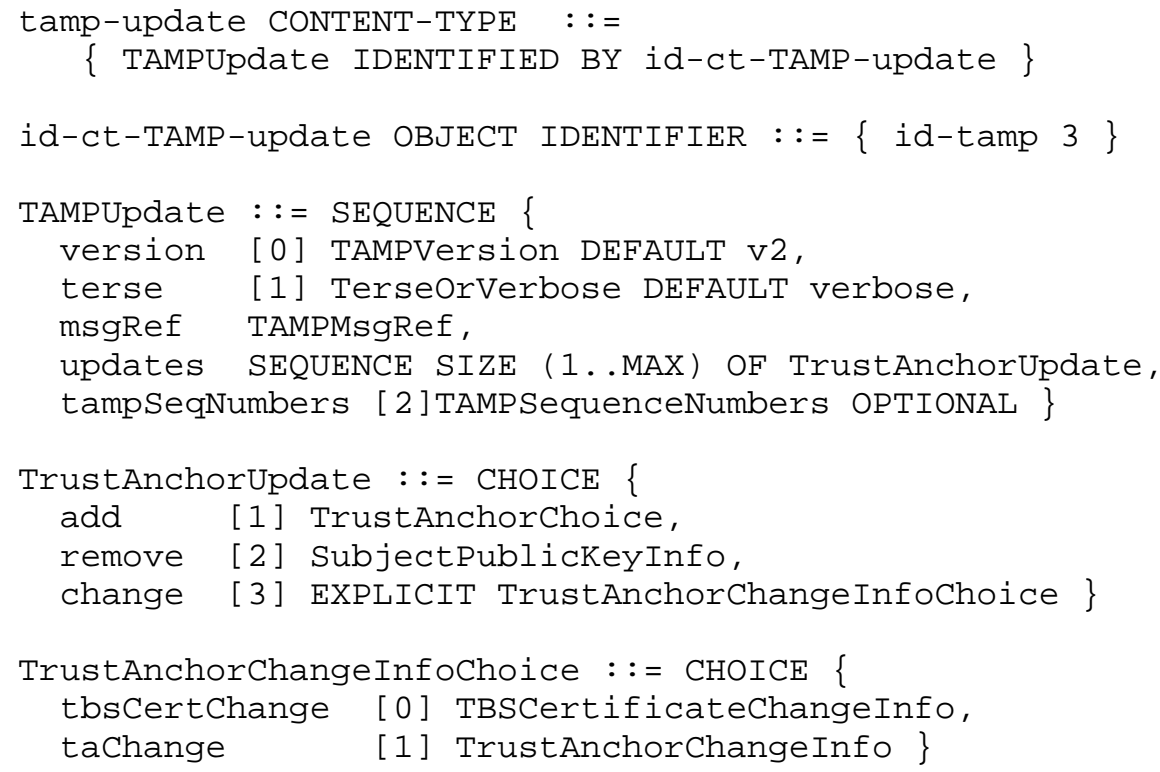




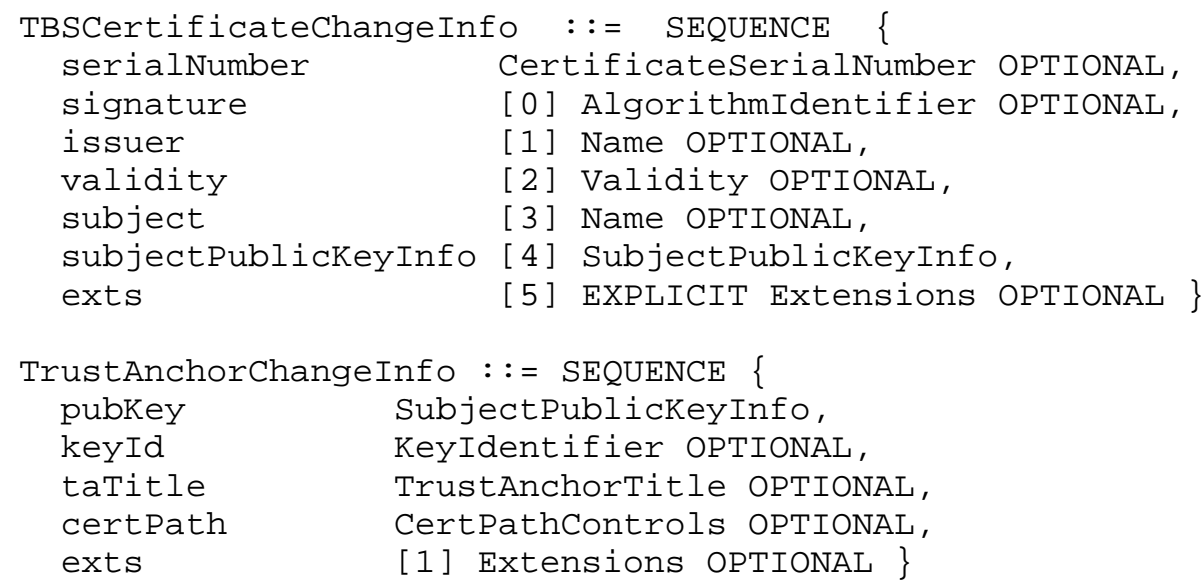

o msgRef contains two items: the target and the seqNum. target identifies the target(s) of the update message. The TargetIdentifier syntax is described in section 4.1. seqNum is a single-use value that will be used to match the Trust Anchor Update message with the Trust Anchor Update Confirm message. The sequence number is also used to detect TAMP message replay. The sequence number processing described in section 6 MUST successfully complete before any of the updates are processed.

- updates contains a sequence of updates, which are used to add, remove, and change management or identity trust anchors. Each entry in the sequence represents one of these actions, and is indicated by an instance of TrustAnchorUpdate. The actions are a batch of updates that MUST be processed in the order that they appear, but each of the updates is processed independently. Each of the updates MUST satisfy the subordination checks described in Section 7. Even if one or more of the updates fail, then the remaining updates MUST be processed. These updates MUST NOT make any changes to the apex trust anchor. 
- tampSeqNumbers MAY be included to provide the initial or new sequence numbers for trust anchors added or changed by the updates field. Elements included in the tampseqNumbers field that do not correspond to an element in the updates field are ignored. Elements included in the tampseqNumbers field that do correspond to an element in the updates field and contain a sequence number less than or equal to the most recently stored sequence number for the trust anchor are ignored. Elements included in the tampseqNumbers field that do correspond to an element in the updates field and contain a sequence number greater than the most recently stored sequence number for the indicated trust anchor are processed by setting the stored sequence number for the trust anchor equal to the new value.

The TrustAnchorUpdate is a choice of three structures, and each alternative represents one of the three possible actions: add, remove, and change. A description of the syntax associated with each of these actions follows:

- add is used to insert a new management or identity trust anchor into the trust anchor store. The TrustAnchorchoice structure is used to provide the trusted public key and all of the information associated with it. However, the action MUST fail with the error code notAuthorized if the subordination checks described in Section 7 are not satisfied. See section 3 for a discussion of the TrustAnchorchoice structure. The apex trust anchor cannot be introduced into a trust anchor store using this action; therefore, the id-pe-wrappedApexContinkey MUST NOT be present in the extensions field. The constraints of the existing trust anchors are unchanged by this action. An attempt to add a management or identity trust anchor that is already in place with the same values for every field in the TrustAnchorChoice structure MUST be treated as a successful addition. An attempt to add a management or identity trust anchor that is already present with the same pubkey values, but with different values for any of the fields in the TrustAnchorchoice structure, MUST fail with the error code improperTAAddition. This means a trust anchor may not be added twice using different TrustAnchorChoice options. If a different format is desired, the existing trust anchor must be removed and the new format added.

o remove is used to delete an existing management or identity trust anchor from the trust anchor store, including the deletion of the management trust anchor associated with the TAMP message signer. However, the action MUST fail with the error code notAuthorized if the subordination checks described in section 7 are not satisfied. The public key contained in subjectPublickeyInfo names the management or identity trust anchor to be deleted. An attempt to 
delete a trust anchor that is not present MUST be treated as a successful deletion. The constraints of the deleted trust anchor are not distributed to other trust anchors in any manner. The apex trust anchor cannot be removed using this action, which ensures that this action cannot place the trust anchor store in an unrecoverable configuration.

- change is used to update the information associated with an existing management or identity trust anchor in the trust anchor store. Attempts to change a trust anchor added as a certificate MUST fail with the error code improperTAChange. The public key contained in the SubjectPublickeyInfo field of TrustAnchorChangeInfo or in the subjectPublickeyInfo field of a TBSCertificatechangeInfo names the to-be-updated trust anchor. However, the action MUST fail with the error code notAuthorized if the subordination checks described in section 7 are not satisfied. An attempt to change a trust anchor that is not present MUST result in a failure with the trustAnchorNotFound status code. The TrustAnchorChangeInfo structure or the TBSCertificatechangeInfo structure is used to provide the revised configuration of the management or identity trust anchor. If the update fails for any reason, then the original trust anchor configuration MUST be preserved. The apex trust anchor information cannot be changed using this action. Attempts to change a trust anchor added as a TBSCertificate using a TrustAnchorChangeInfo MUST fail with an improperTAChange error. Attempts to change a trust anchor added as a TrustAnchorInfo using a TBSCertificateChangeInfo MUST fail with an improperTAChange error.

The fields of TrustAnchorChangeInfo are used as follows:

- pubkey contains the algorithm identifier and the public key of the management or identity trust anchor. It is used to locate the to-be-updated trust anchor in the trust anchor store.

o keyId is OPTIONAL, and when present, it contains the public key identifier of the trust anchor public key, which is contained in the pubKey field. If this field is not present, then the public key identifier remains unchanged. If this field is present, the provided public key identifier replaces the previous one.

o taTitle is OPTIONAL, and when present, it provides a human readable name for the management or identity trust anchor. When absent in a change trust anchor update, any title that was previously associated with the trust anchor is removed. Similarly, when present in a change trust anchor update, the title 
in the message is associated with the trust anchor. If a previous title was associated with the trust anchor, then the title is replaced. If a title was not previously associated with the trust anchor, then the title from the update message is added.

- certPath is OPTIONAL, and when present, it provides the controls needed to construct and validate an X.509 certification path. When absent in a change trust anchor update, any controls that were previously associated with the management or identity trust anchor are removed, which means that delegation is no longer permitted. Similarly, when present in a change trust anchor update, the controls in the message are associated with the management or identity trust anchor. If previous controls, including the trust anchor distinguished name, were associated with the trust anchor, then the controls are replaced, which means that delegation continues to be supported, but that different certification paths will be valid. If controls were not previously associated with the management or identity trust anchor, then the controls from the update message are added, which enables delegation. The syntax and semantics of CertPathcontrols are discussed in [RFC5914].

o exts is OPTIONAL, and when present, it provides the extensions values that are associated with the trust anchor. When absent in a change trust anchor update, any extensions that were previously associated with the trust anchor are removed. Similarly, when present in a change trust anchor update, the extensions in the message are associated with the trust anchor. Any extensions previously associated with the trust anchor are replaced or removed.

The fields of TBSCertificatechangeInfo are used to alter the fields within a TBSCertificate structure. TBSCertificate is described in [RFC5280]. For all fields except exts, if the field is absent in a change trust anchor update, then any previous value associated with a trust anchor is unchanged. For the exts field, if the field is absent in a change trust anchor update, then any previous value associated with a trust anchor is removed. For all fields, if the field is present in a change trust anchor update, then any previous value associated with a trust anchor is replaced with the value from the update message.

\subsubsection{Trust Anchor List}

[RFC5914] defines the TrustAnchorList structure to convey a list of trust anchors. TAMP implementations MAY process TrustAnchorList objects (with econtentType (or contentType) using the id-cttrustAnchorList OID defined in [RFC5914]) as equivalent to TAMPUpdate 
objects with terse set to terse, msgRef set to allModules (with a suitable sequence number), and all elements within the list contained within the add field. This alternative to TrustAnchorUpdate is provided for implementations that perform integrity and authorization checks out-of-band as a simple means of transferring trust anchors from one trust anchor store to another. It does not provide a means of removing or changing trust anchors and has no HTTP binding.

\subsection{Trust Anchor Update Confirm}

The Trust Anchor Update Confirm message is a reply by a trust anchor store to a valid Trust Anchor Update message. The Trust Anchor Update Confirm message provides success and failure information for each of the requested updates. The Trust Anchor Update Confirm message MAY be signed or unsigned. A Trust Anchor Update Confirm message MUST be signed if the implementation is capable of signing it.

The Trust Anchor Update Confirm content type has the following syntax:

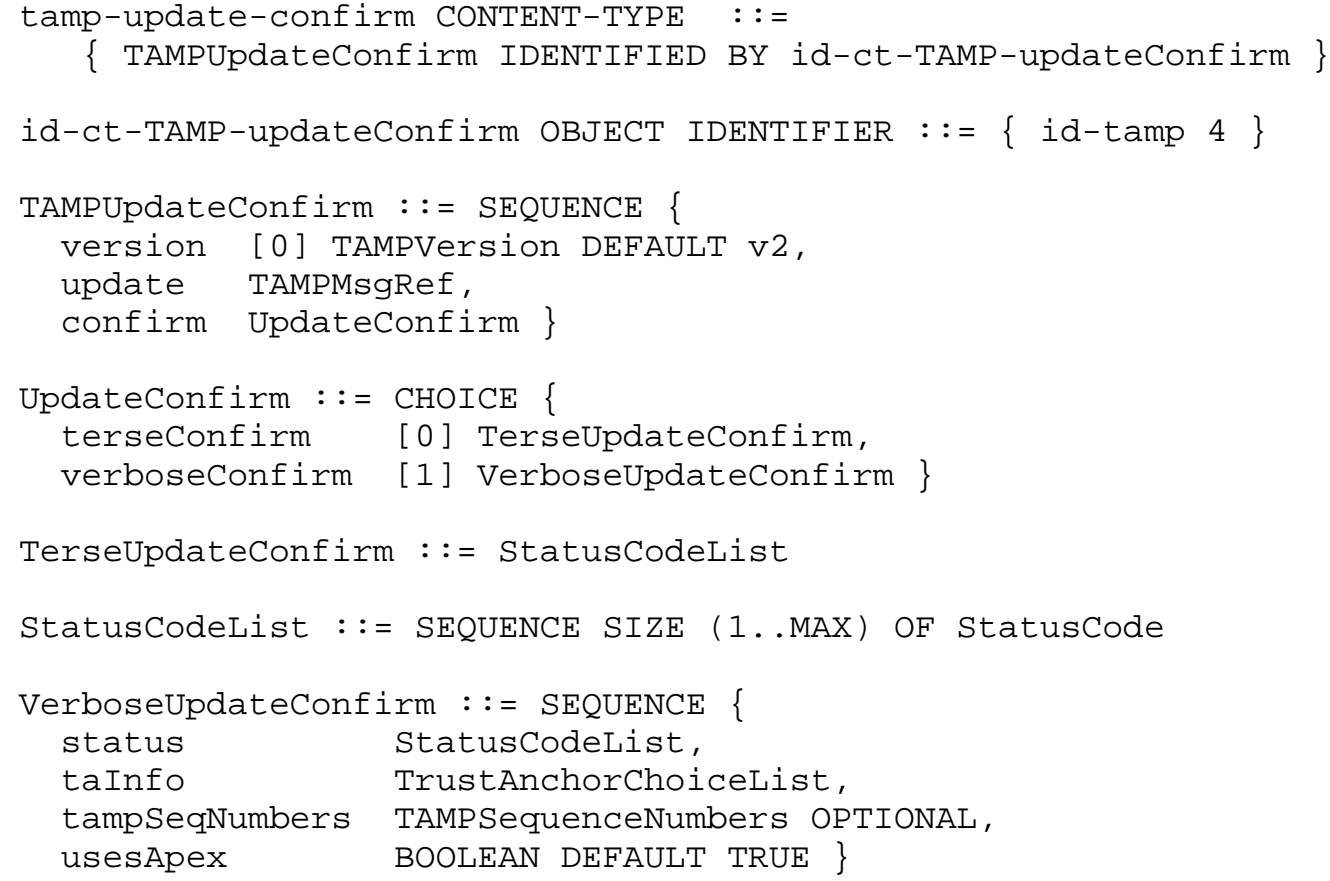


The fields of TAMPUpdateConfirm are used as follows:

o version identifies version of TAMP. For this version of the specification, the default value, v2, MUST be used.

- update identifies the TAMPUpdate message to which the trust anchor store is responding. The update structure repeats the TAMPMsgRef from the Trust Anchor Update message (see Section 4.3). The sequence number processing described in section 6 MUST successfully complete before any of the updates are processed.

- confirm contains either a terse update confirmation or a verbose update confirmation. The terse update confirmation is represented by TerseUpdateConfirm, and the verbose response is represented by VerboseUpdateConfirm.

The TerseUpdateConfirm contains a sequence of status codes, one for each TrustAnchorUpdate structure in the Trust Anchor Update message. The status codes MUST appear in the same order as the TrustAnchorUpdate structures to which they apply, and the number of elements in the status code list MUST be the same as the number of elements in the trust anchor update list. Each of the status codes is discussed in section 5.

The fields of VerboseUpdateConfirm are used as follows:

o status contains a sequence of status codes, one for each TrustAnchorUpdate structure in the Trust Anchor Update message. The status codes appear in the same order as the TrustAnchorUpdate structures to which they apply, and the number of elements in the status code list MUST be the same as the number of elements in the trust anchor update list. Each of the status codes is discussed in Section 5 .

- taInfo contains a sequence of TrustAnchorChoice structures. One entry in the sequence is provided for each trust anchor contained in the trust anchor store. These represent the state of the trust anchors after the updates have been processed. When usesApex is true, the apex trust anchor is the first trust anchor in the sequence.

- tampseqNumbers is used to indicate the currently held sequence number for each trust anchor authorized to sign TAMP messages. The keyId field identifies the trust anchor, and the seqNumber field provides the current sequence number associated with the trust anchor. 
- usesApex is a Boolean value that indicates whether the first item in the taInfo field identifies the apex TA.

\subsection{Apex Trust Anchor Update}

The Apex Trust Anchor Update message replaces the operational public key and, optionally, the contingency public key associated with the apex trust anchor. Each trust anchor store has exactly one apex trust anchor. No constraints are associated with the apex trust anchor. The public key identifier of the operational public key is used to identify the apex trust anchor in subsequent TAMP messages. The digital signature on the Apex Trust Anchor Update message is validated with either the current operational public key or the current contingency public key. For the Apex Trust Anchor Update message that is validated with the operational public key to be valid, the trust anchor store MUST be a target of the update, the sequence number MUST be larger than the most recently stored sequence number for the operational public key, and the digital signature MUST be validated directly with the operational public key. That is, no delegation via a certification path is permitted. For the Apex Trust Anchor Update message that is validated with the contingency public key to be valid, the trust anchor store MUST be a target of the update, the provided decryption key MUST properly decrypt the contingency public key, and the digital signature MUST be validated directly with the decrypted contingency public key. Again, no delegation via a certification path is permitted.

If the Apex Trust Anchor Update message is validated using the operational public key, then sequence number processing is handled normally, as described in Section 6. If the Apex Trust Anchor Update message is validated using the contingency public key, then the TAMPMsgRef sequence number MUST contain a zero value. A sequence number for subsequent messages that will be validated with the new operational public key can optionally be provided. If no value is provided, then the trust anchor store MUST be prepared to accept any sequence number in the next TAMP message validated with the newly installed apex trust anchor operational public key. If the Apex Trust Anchor Update message is valid and the clearTrustAnchors flag is set to TRUE, then all of the management and identity trust anchors stored in the trust anchor store MUST be deleted. That is, the new apex trust anchor MUST be the only trust anchor remaining in the trust anchor store. If the Apex Trust Anchor Update message is valid and the clearCommunities flag is set to TRUE, then all community identifiers stored in the trust anchor store MUST be deleted.

The SignedData structure includes a SignerInfo.sid value, and it identifies the apex trust anchor public key that will be used to validate the digital signature on this TAMP message. The public key 
identifier for the operational public key is known in advance, and it is stored as part of the apex trust anchor. The public key identifier for the contingency public key is not known in advance; however, the presence of the unsigned attribute containing the symmetric key needed to decrypt the contingency public key unambiguously indicates that the TAMP message signer used the contingency private key to sign the Apex Trust Anchor Update message.

If the digital signature on the Apex Trust Anchor Update message is valid using either the apex trust anchor operational public key or the apex trust anchor contingency public key, sequence number checking is successful, and the trust anchor store is an intended recipient of the TAMP message, then the trust anchor store MUST update the apex trust anchor and return an Apex Trust Anchor Update Confirm message. If an Apex Trust Anchor Update Confirm message is not returned, then a TAMP Error message SHOULD be returned. Note that the sequence number MUST be zero if the Apex Trust Anchor Update message is validated with the apex trust anchor contingency public key.

The Apex Trust Anchor Update content type has the following syntax:

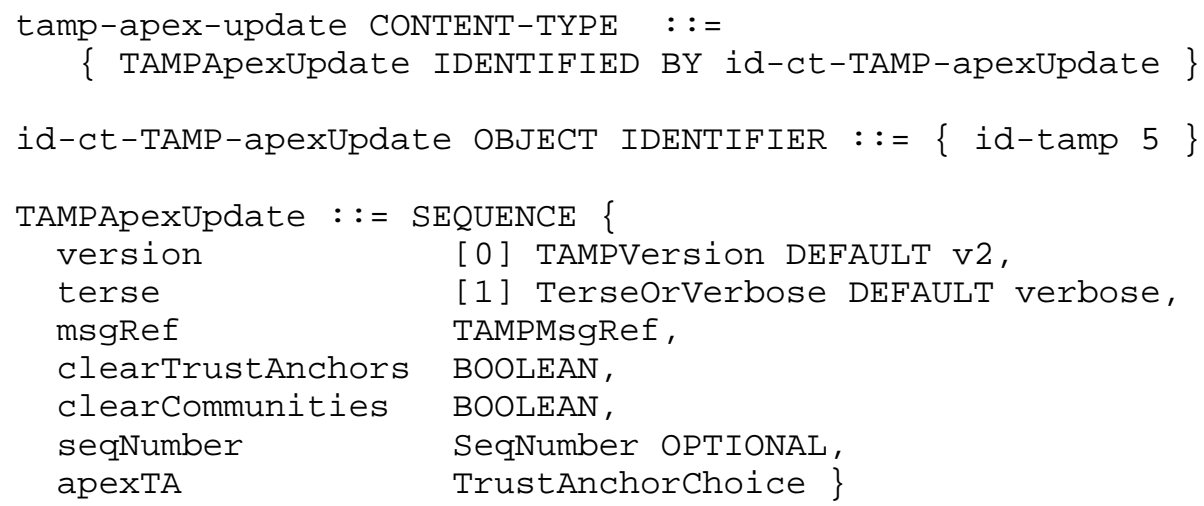

The fields of TAMPApexUpdate are used as follows:

- version identifies version of TAMP. For this version of the specification, the default value, v2, MUST be used.

- terse indicates the type of response that is desired. A terse response is indicated by a value of 1 , and a verbose response is indicated by a value of 2 , which is omitted during encoding since it is the default value.

- msgRef contains two items: the target and the seqNum. target identifies the target(s) of the Apex Trust Anchor Update message. 
The TargetIdentifier syntax as described in section 4.1 is used. seqNum is a single-use value that will be used to match the Apex Trust Anchor Update message with the Apex Trust Anchor Update Confirm message. The sequence number is also used to detect TAMP message replay if the message is validated with the apex trust anchor operational public key. The sequence number processing described in section 6 MUST successfully complete before any action is taken. However, seqNum MUST contain a zero value if the message is validated with the apex trust anchor contingency public key.

- clearTrustAnchors is a Boolean. If the value is set to TRUE, then all of the management and identity trust anchors stored in the trust anchor store MUST be deleted, leaving the newly installed apex trust anchor as the only trust anchor in the trust anchor store. If the value is set to FALSE, the other trust anchors MUST NOT be changed.

- clearCommunities is a Boolean. If the value is set to TRUE, then all of the community identifiers stored in the trust anchor store MUST be deleted, leaving none. If the value is set to FALSE, the list of community identifiers MUST NOT be changed.

o seqNumber is OPTIONAL, and when present, it provides the initial sequence number for the apex trust anchor. If seqNumber is absent, the trust anchor store is prepared to accept any sequence number value for the apex trust anchor operational public key.

- apextA provides the information for the replacement apex trust anchor. The TrustAnchorchoice structure is used to provide the trusted public key and all of the information associated with it. The pubKey, keyId, taTitle, certPath, and exts fields apply to the operational public key of the apex trust anchor. The ApexTrustAnchorInfo certificate extension MAY appear as an extension. Section 9 describes the WrappedApexContingencykey certificate extension.

\subsection{Apex Trust Anchor Update Confirm}

The Apex Trust Anchor Update Confirm message is a reply by a trust anchor store to a valid Apex Trust Anchor Update message. The Apex Trust Anchor Update Confirm message provides success or failure information for the apex trust anchor update. The Apex Trust Anchor Update Confirm message MAY be signed or unsigned. An Apex Trust Anchor Update Confirm message MUST be signed if the trust anchor store is capable of signing it. 
The Apex Trust Anchor Update Confirm content type has the following syntax:

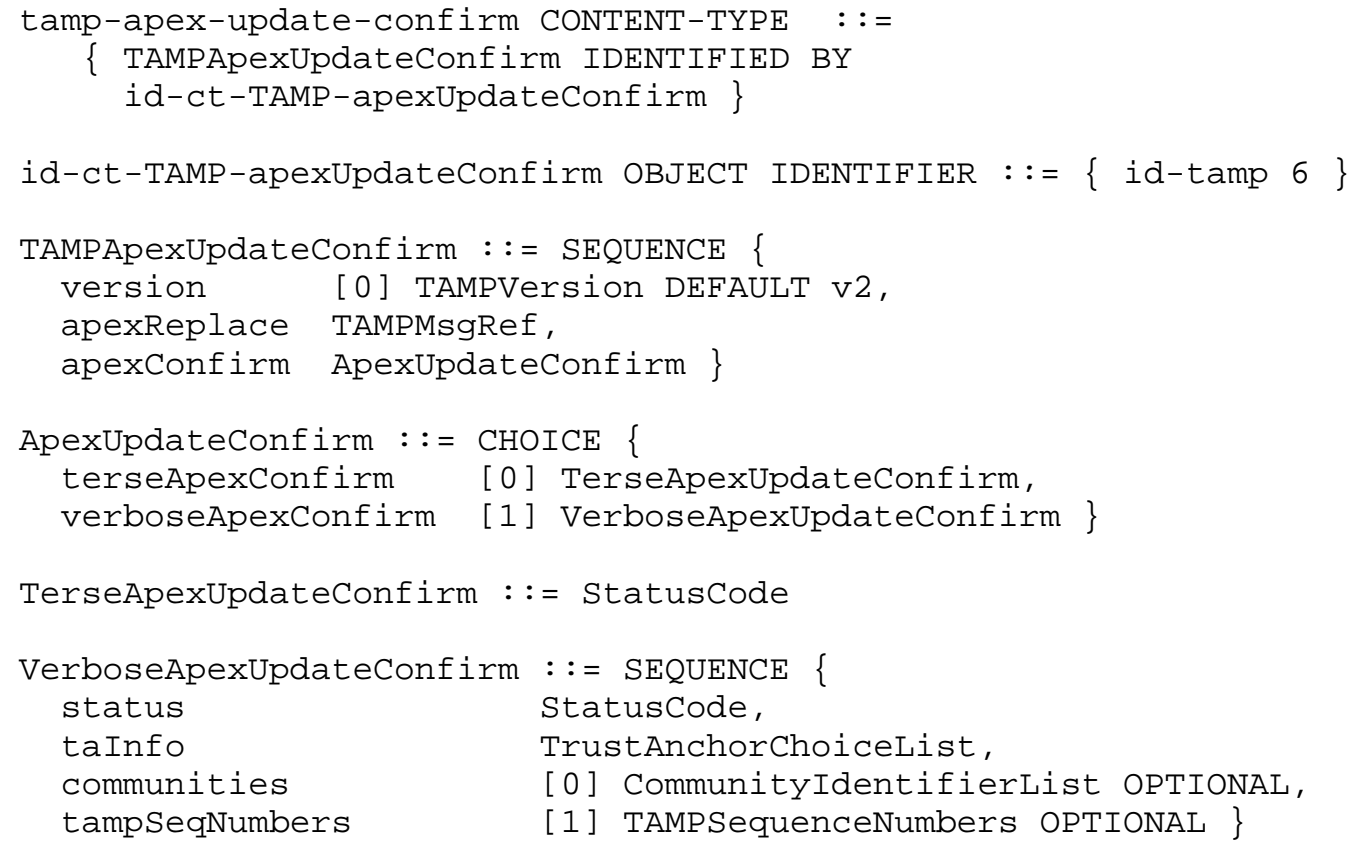

The fields of TAMPApexUpdateConfirm are used as follows:

- version identifies version of TAMP. For this version of the specification, the default value, v2, MUST be used.

- apexReplace identifies the Apex Trust Anchor Update message to which the trust anchor store is responding. The apexReplace structure repeats the TAMPMsgRef from the beginning of the Apex Trust Anchor Update message (see Section 4.5). When the Apex Trust Anchor Update message is validated with the operational public key, the sequence number processing described in section 6 MUST successfully complete before an Apex Trust Anchor Update Confirm message is generated. When the Apex Trust Anchor Update message is validated with the contingency public key, normal sequence number processing is ignored, but the seqNum MUST be zero.

- apexConfirm contains either a terse update confirmation or a verbose update confirmation. The terse update confirmation is represented by TerseApexUpdateConfirm, and the verbose response is represented by VerboseApexUpdateConfirm. 
The TerseApexUpdateConfirm contains a single status code, indicating the success or failure of the apex trust anchor update. If the apex trust anchor update failed, then the status code provides the reason for the failure. Each of the status codes is discussed in section 5.

The fields of VerboseApexUpdateConfirm are used as follows:

- status contains a single status code, indicating the success or failure of the apex trust anchor update. If the apex trust anchor update failed, then the status code provides the reason for the failure. Each of the status codes is discussed in section 5 .

- taInfo contains a sequence of TrustAnchorChoice structures. One entry in the sequence is provided for each trust anchor contained in the trust anchor store. These represent the state of the trust anchors after the apex trust anchor update has been processed. See [RFC5914] for a description of the TrustAnchorInfo structure. The apex trust anchor is the first trust anchor in the sequence.

- communities is OPTIONAL. When present, it contains a sequence of object identifiers. Each object identifier names one community to which this trust anchor store belongs. When the trust anchor store belongs to no communities, this field is omitted.

- tampseqNumbers is used to indicate the currently held sequence number for each trust anchor authorized to sign TAMP messages. The keyId field identifies the trust anchor, and the seqNumber field provides the current sequence number associated with the trust anchor.

\subsection{Community Update}

The trust anchor store maintains a list of identifiers for the communities of which it is a member. The Community Update message can be used to remove or add community identifiers from this list. The Community Update message MUST be signed. For the Community Update message to be valid, the trust anchor store MUST be a target of the update; the sequence number checking described in Section 6 MUST be successful when the TAMP message signer is a trust anchor; and the digital signature MUST be validated by the apex trust anchor operational public key, an authorized management trust anchor, or via an authorized X.509 certification path originating with such a trust anchor.

If the trust anchor store supports the Community Update message, the digital signature on the Community Update message is valid, sequence number checking is successful, the signer is authorized, and the trust anchor store is an intended recipient of the TAMP message, then 
the trust anchor store MUST make the specified updates and return a Community Update Confirm message. If a Community Update Confirm message is not returned, then a TAMP Error message SHOULD be returned.

The Community Update message contains a batch of updates, and all of the updates MUST be accepted for the trust anchor store to return a successful Community Update Confirm message. The remove updates, if present, MUST be processed before the add updates. Where remove is present with an empty list, all community identifiers MUST be removed. This approach prevents community identifiers that are intended to be mutually exclusive from being installed by a successful addition and a failed removal. Where add is present, at least one community identifier MUST appear in the list.

The Community Update content type has the following syntax:

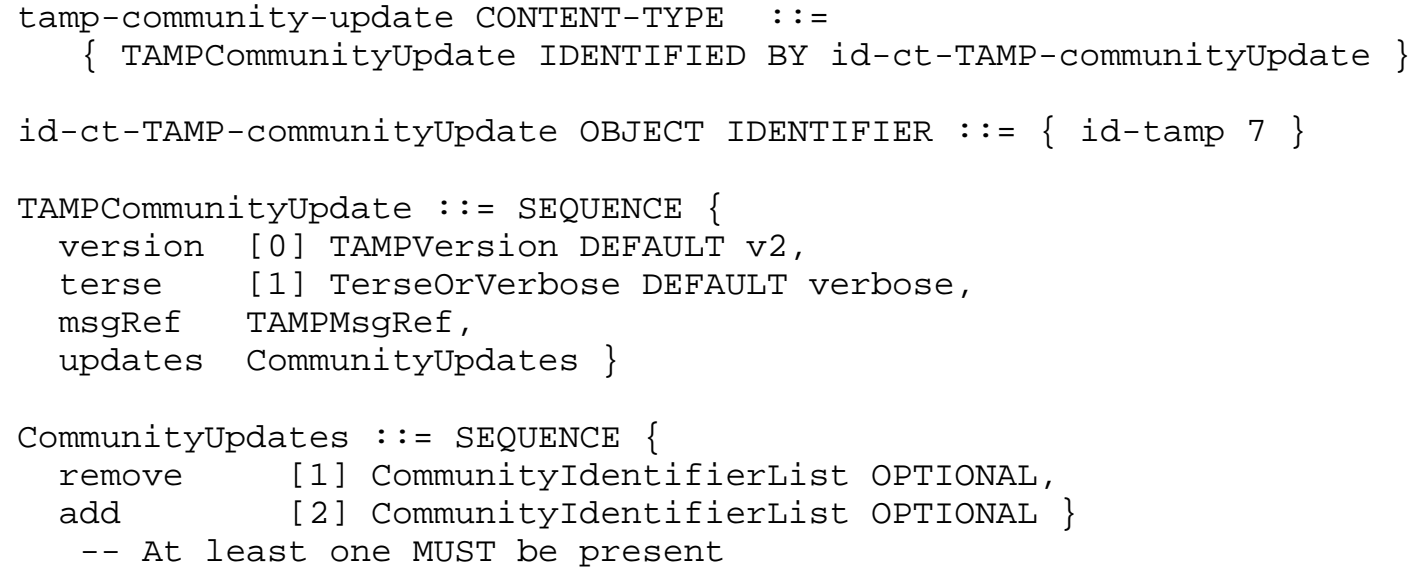

The fields of TAMPCommunityUpdate are used as follows:

- version identifies version of TAMP. For this version of the specification, the default value, v2, MUST be used.

- terse indicates the type of response that is desired. A terse response is indicated by a value of 1 , and a verbose response is indicated by a value of 2 , which is omitted during encoding since it is the default value.

o msgRef contains two items: the target and the segNum. target identifies the target(s) of the update message. The TargetIdentifier syntax as described in section 4.1 is used. seqNum is a single-use value that will be used to match the Community Update message with the Community Update Confirm 
message. The sequence number is also used to detect TAMP message replay. The sequence number processing described in section 6 MUST successfully complete before any of the updates are processed.

- updates contains a sequence of community identifiers to be removed and a sequence of community identifiers to be added. These are represented by the Communityupdates structure.

The Communityupdates is a sequence of two OPTIONAL sequences, but at least one of these sequences MUST be present. The first sequence contains community identifiers to be removed, and if there are none, it is absent. Where remove is present with an empty list, all community identifiers MUST be removed. The second sequence contains community identifiers to be added, and if there are none, it is absent. The remove updates, if present, MUST be processed before the add updates. An error is generated if any of the requested removals or additions cannot be accomplished. However, requests to remove community identifiers that are not present are treated as successful removals. Likewise, requests to add community identifiers that are already present are treated as successful additions. If an error is generated, the trust anchor store community list MUST NOT be changed.

A description of the syntax associated with each of these actions follows:

- remove is used to remove one, multiple, or all community identifiers from the trust anchor store.

- add is used to insert one or more new community identifiers into the trust anchor store.

\subsection{Community Update Confirm}

The Community Update Confirm message is a reply by a trust anchor store to a valid Community Update message. The Community Update Confirm message provides success or failure information for the requested updates. Success is returned only if the whole batch of updates is successfully processed. If any of the requested updates cannot be performed, then a failure is indicated, and the set of community identifiers stored in the trust anchor store is unchanged. The Community Update Confirm message MAY be signed or unsigned. A Community Update Confirm message MUST be signed if the trust anchor store is capable of signing it. 
The Community Update Confirm content type has the following syntax:

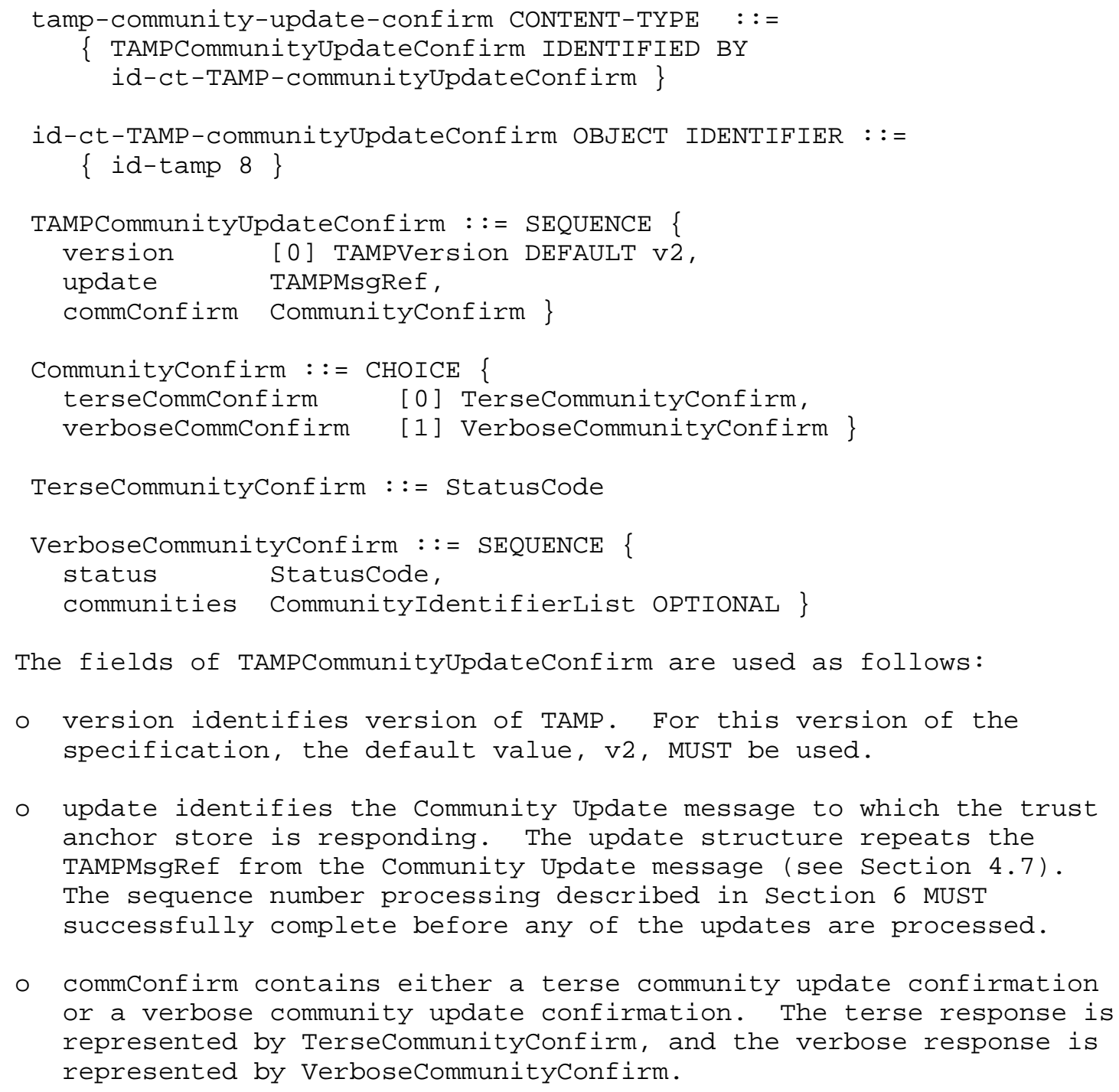


The fields of VerbosecommunityConfirm are used as follows:

- status contains a single status code, indicating the success or failure of the Community Update message processing. If the community update failed, then the status code indicates the reason for the failure. Each of the status codes is discussed in Section 5 .

- communities is OPTIONAL. When present, it contains the sequence of community identifiers present in the trust anchor store after the update is processed. When the trust anchor store belongs to no communities, this field is omitted.

4.9. Sequence Number Adjust

The trust anchor store maintains the current sequence number for the apex trust anchor and each management trust anchor authorized for TAMP messages. Sequence number processing is discussed in section 6 . The Sequence Number Adjust message can be used to provide the most recently used sequence number to one or more targets, thereby reducing the possibility of replay. The sequence Number Adjust message MUST be signed. For the Sequence Number Adjust message to be valid, the trust anchor store MUST be an intended recipient of the Sequence Number Adjust message, the sequence number MUST be equal to or larger than the most recently stored sequence number for the originating trust anchor, and the digital signature MUST be validated by the apex trust anchor operational public key or an authorized management trust anchor.

If the digital signature on the Sequence Number Adjust message is valid, the sequence number is equal to or larger than the most recently stored sequence number for the originating trust anchor, the signer is authorized, and the trust anchor store is an intended recipient of the TAMP message, then the trust anchor store MUST update the sequence number associated with the originating trust anchor and return a Sequence Number Adjust Confirm message. If a Sequence Number Adjust Confirm message is not returned, then a TAMP Error message SHOULD be returned.

The Sequence Number Adjust message contains an adjustment for the sequence number of the TAMP message signer. 
The Sequence Number Adjust content type has the following syntax:

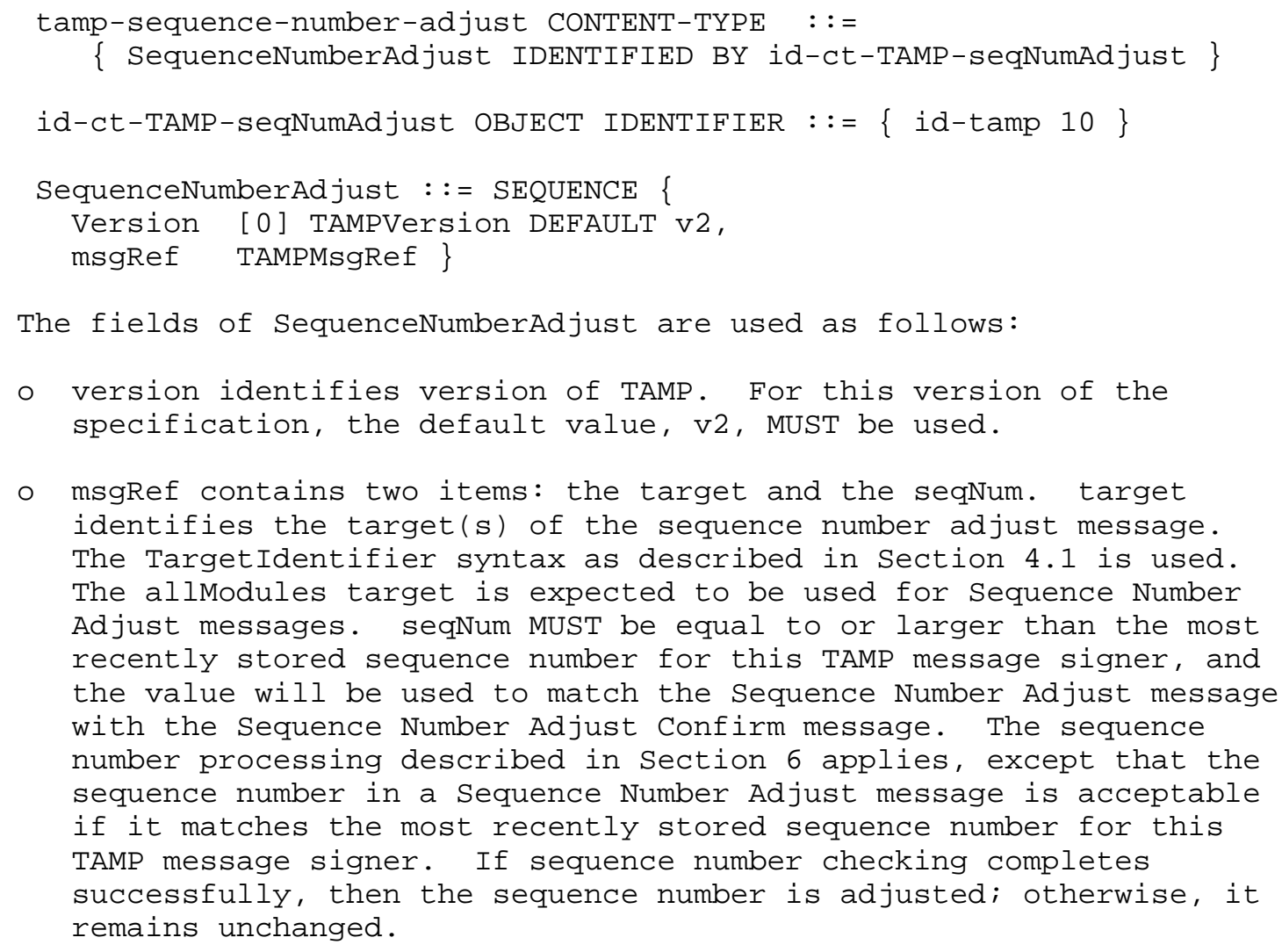

The Sequence Number Adjust Confirm message is a reply by a trust anchor store to a valid Sequence Number Adjust message. The Sequence Number Adjust Confirm message provides success or failure information. Success is returned only if the sequence number for the trust anchor that signed the Sequence Number Adjust message originator is adjusted. If the sequence number cannot be adjusted, then a failure is indicated, and the sequence number stored in the trust anchor store is unchanged. The Sequence Number Adjust Confirm message MAY be signed or unsigned. A Sequence Number Adjust Confirm message MUST be signed if the trust anchor store is capable of signing it. 
The Sequence Number Adjust Confirm content type has the following syntax:

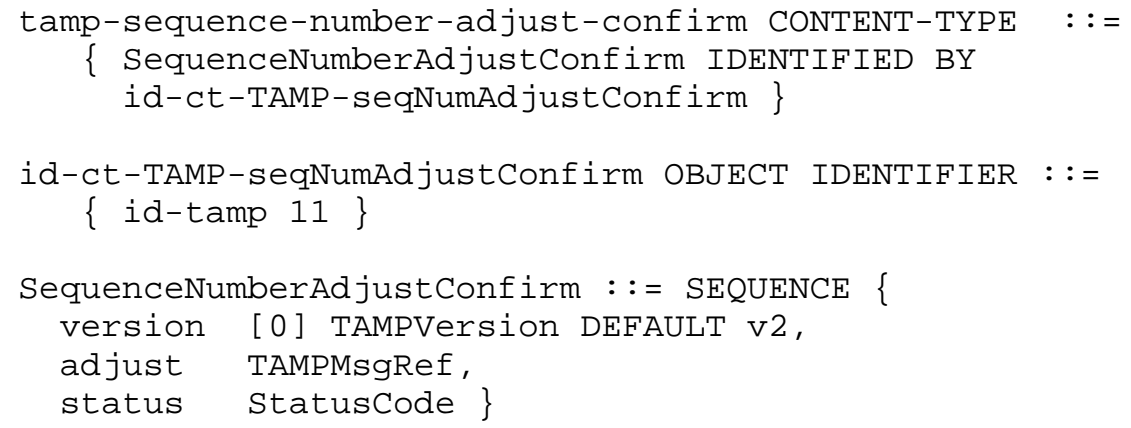

The fields of SequenceNumberAdjustConfirm are used as follows:

- version identifies version of TAMP. For this version of the specification, the default value, v2, MUST be used.

- adjust identifies the Sequence Number Adjust message to which the trust anchor store is responding. The adjust structure repeats the TAMPMsgRef from the Sequence Number Adjust message (see Section 4.9). The sequence number processing described in Section 6 MUST successfully complete to adjust the sequence number associated with the sequence Number Adjust message originator.

- status contains a single status code, indicating the success or failure of the sequence Number Adjust message processing. If the adjustment failed, then the status code indicates the reason for the failure. Each of the status codes is discussed in Section 5.

\subsection{TAMP Error}

The TAMP Error message is a reply by a trust anchor store to any invalid TAMP message. The TAMP Error message provides an indication of the reason for the error. The TAMP Error message MAY be signed or unsigned. A TAMP Error message MUST be signed if the trust anchor store is capable of signing it. For the request types defined in this specification, TAMP Error messages MUST NOT be used to indicate a request message was successfully processed. Each TAMP Error message identifies the type of TAMP message that caused the error. In cases where the TAMP message type cannot be determined, errors MAY be returned via other means, such as at the protocol level, via an attached display, etc. 
The TAMP Error message content type has the following syntax:

tamp-error CONTENT-TYPE : :=

\{ TAMPError IDENTIFIED BY id-ct-TAMP-error \}

id-ct-TAMP-error OBJECT IDENTIFIER : := \{id-tamp 9$\}$

TAMPError : := SEQUENCE \{

version [0] TAMPVersion DEFAULT v2,

msgType OBJECT IDENTIFIER,

status Statuscode,

msgRef TAMPMsgRef OPTIONAL \}

The fields of TAMPError are used as follows:

- version identifies version of TAMP. For this version of the specification, the default value, v2, MUST be used.

- msgType indicates the content type of the TAMP message that caused the error.

- status contains a status code that indicates the reason for the error. Each of the status codes is discussed in section 5.

- msgref is OPTIONAL, but whenever possible it SHOULD be present. It identifies the TAMP message that caused the error. It repeats the target and seqNum from the TAMP message that caused the error (see Sections 4.1,4.3, 4.5,4.7, and 4.9).

5. Status Codes

The Trust Anchor Update Confirm, the Apex Trust Anchor Update Confirm, the Community Update Confirm, the Sequence Number Adjust Confirm, and the TAMP Error messages include status codes. The syntax for the status codes is:

$\begin{array}{ll}\text { StatusCode : := ENUMERATED } & \\ \text { success } & (0), \\ \text { decodeFailure } & (1), \\ \text { badContentInfo } & (2), \\ \text { badSignedData } & (3), \\ \text { badEncapContent } & (4), \\ \text { badCertificate } & (5), \\ \text { badSignerInfo } & (6), \\ \text { badSignedAttrs } & (7), \\ \text { badUnsignedAttrs } & (8), \\ \text { missingContent } & (9), \\ \text { noTrustAnchor } & (10),\end{array}$




$\begin{array}{lr}\text { notAuthorized } & (11), \\ \text { badDigestAlgorithm } & (12), \\ \text { badSignatureAlgorithm } & (13), \\ \text { unsupportedKeySize } & (14), \\ \text { unsupportedParameters } & (15), \\ \text { signatureFailure } & (16), \\ \text { insufficientMemory } & (17), \\ \text { unsupportedTAMPMsgType } & (18), \\ \text { apexTAMPAnchor } & (19), \\ \text { improperTAAddition } & (20), \\ \text { segNumFailure } & (21), \\ \text { contingencyPublickeyDecrypt } & (22), \\ \text { incorrectTarget } & (23), \\ \text { communityUpdateFailed } & (24), \\ \text { trustAnchorNotFound } & (25), \\ \text { unsupportedTAAlgorithm } & (26), \\ \text { unsupportedTAKeySize } & (27), \\ \text { unsupportedContinPubKeyDecryptAlg } & (28), \\ \text { missingSignature } & (29), \\ \text { resourcesBusy } & (30), \\ \text { versionNumberMismatch } & (31), \\ \text { missingPolicySet } & (32), \\ \text { revokedCertificate } & (33), \\ \text { unsupportedTrustAnchorFormat } & (34), \\ \text { improperTAChange } & (35), \\ \text { malformed } & (36), \\ \text { cmsError } & (37), \\ \text { unsupportedTargetIdentifier } & (38), \\ \text { other } & (127), \\ & \end{array}$

The various values of Statuscode are used as follows:

- success is used to indicate that an update, portion of an update, or adjust was processed successfully.

- decodefailure is used to indicate that the trust anchor store was unable to successfully decode the provided message. The specified content type and the provided content do not match.

- badContentInfo is used to indicate that the ContentInfo syntax is invalid or that the contentType carried within the contentInfo is unknown or unsupported.

- badSignedData is used to indicate that the SignedData syntax is invalid, the version is unknown or unsupported, or more than one entry is present in digestAlgorithms. 
- badEncapContent is used to indicate that the EncapsulatedContentInfo syntax is invalid. This error can be generated due to problems located in SignedData.

- badCertificate is used to indicate that the syntax for one or more certificates in Certificateset is invalid.

- badSignerInfo is used to indicate that the SignerInfo syntax is invalid, or the version is unknown or unsupported.

- badSignedAttrs is used to indicate that the signedAttrs syntax within SignerInfo is invalid.

- badUnsignedAttrs is used to indicate that the unsignedAttrs syntax within SignerInfo is invalid.

- missingContent is used to indicate that the OPTIONAL eContent is missing in EncapsulatedContentInfo, which is REQUIRED in this specification. This error can be generated due to problems located in SignedData.

- notrustAnchor is used to indicate one of two possible error situations. In one case, the subjectKeyIdentifier does not identify the public key of a trust anchor or a certification path that terminates with an installed trust anchor. In the other case, the issuerAndSerialNumber is used to identify the TAMP message signer, which is prohibited by this specification.

- notAuthorized is used to indicate one of two possible error situations. In one case, the sid within SignerInfo leads to an installed trust anchor, but that trust anchor is not an authorized signer for the received TAMP message content type. Identity trust anchors are not authorized signers for any of the TAMP message content types. In the other case, the signer of a Trust Anchor Update message is not authorized to manage the to-be-updated trust anchor as determined by a failure of the subordination processing in Section 7.

- badDigestAlgorithm is used to indicate that the digestAlgorithm in either SignerInfo or SignedData is unknown or unsupported.

- badSignatureAlgorithm is used to indicate that the signatureAlgorithm in SignerInfo is unknown or unsupported.

- unsupportedKeysize is used to indicate that the signatureAlgorithm in SignerInfo is known and supported, but the TAMP message digital signature could not be validated because an unsupported key size was employed by the signer. 
o unsupportedParameters is used to indicate that the signatureAlgorithm in SignerInfo is known, but the TAMP message digital signature could not be validated because unsupported parameters were employed by the signer.

- signatureFailure is used to indicate that the signatureAlgorithm in SignerInfo is known and supported, but the digital signature in the signature field within SignerInfo could not be validated.

- insufficientMemory indicates that the update could not be processed because the trust anchor store did not have sufficient memory to store the resulting trust anchor configuration or community identifier.

- unsupportedTAMPMsgType indicates that the TAMP message could not be processed because the trust anchor store does not support the provided TAMP message type. This code will be used if the id-ct-TAMP-communityUpdate content type is provided and the trust anchor store does not support the Community Update message. This status code will also be used if the contentType value within econtent Type is not one that is defined in this specification.

- apexTAMPAnchor indicates that the update could not be processed because the Trust Anchor Update message tried to remove the apex trust anchor.

- improperTAAddition indicates that a trust anchor update is trying to add a new trust anchor that may already exist, but some attributes of the to-be-added trust anchor are being modified in an improper manner. The desired trust anchor configuration may be attainable with a change operation instead of an add operation.

- seqNumFailure indicates that the TAMP message could not be processed because the processing of the sequence number, which is described in section 6, resulted in an error.

- contingencyPublickeyDecrypt indicates that the update could not be processed because an error occurred while decrypting the contingency public key.

- incorrectTarget indicates that the query, update, or adjust message could not be processed because the trust anchor store is not the intended recipient.

- communityupdateFailed indicates that the community update requested the addition of a community identifier or the removal of a community identifier, but the request could not be honored. 
- trustAnchorNotFound indicates that a change to a trust anchor was requested, but the referenced trust anchor is not represented in the trust anchor store.

o unsupportedTAAlgorithm indicates that an update message would result in the trust anchor with a public key associated with a digital signature validation algorithm that is not implemented. In addition, this status code is used if the algorithm is supported, but the parameters associated with the algorithm are not supported.

- unsupportedTAKeySize indicates that the trust anchor would include a public key of a size that is not supported.

- unsupportedContinPubKeyDecryptAlg indicates that the decryption algorithm for the apex trust anchor contingency public key is not supported.

- missingsignature indicates that an unsigned TAMP message was received, but the received TAMP message type MUST be signed.

- resourcesBusy indicates that the resources necessary to process the TAMP message are not available at the present time, but the resources might be available at some point in the future.

- versionNumberMismatch indicates that the version number in a received TAMP message is not acceptable.

- missingPolicyset indicates that the policyFlags associated with a trust anchor are set in a fashion that requires the policyset to be present, but the policyset is missing.

- revokedCertificate indicates that one or more of the certificates needed to properly process the TAMP message have been revoked.

- unsupportedTrustAnchorFormat indicates that an unsupported trust anchor format was presented or the version is unknown or unsupported.

- improperTAChange indicates that a trust anchor update is trying to change a new trust anchor using a format different than the format of the existing trust anchor.

- malformed indicates an error in the composition of the CMS structure encapsulating a TAMP message. 
- cmsError indicates an error processing a CMS structure that encapsulated a TAMP message, such as an error processing Content Type or MessageDigest attributes.

- unsupportedTargetIdentifier indicates that a msgRef with an unsupported TargetIdentifier option was encountered.

- other indicates that the update could not be processed, but the reason is not covered by any of the assigned status codes. Use of this status code SHOULD be avoided.

6. Sequence Number Processing

The sequence number processing facilities in TAMP represent a balance between replay protection, operational considerations, and trust anchor store memory management. The goal is to provide replay protection without making TAMP difficult to use, creating an environment where surprising error conditions occur on a regular basis, or imposing onerous memory management requirements on implementations. This balance is achieved by performing sequence number checking on TAMP messages that are validated directly using a trust anchor, and allowing these checks to be skipped whenever the TAMP message originator is not represented by a trust anchor. Implementations MUST perform sequence number checking on TAMP messages that are validated directly using a trust anchor and MAY perform sequence number checking for TAMP messages validated using a certification path.

The TAMP Status Query, Trust Anchor Update, Apex Trust Anchor Update, Community Update, and Sequence Number Adjust messages include a sequence number. This single-use identifier is used to match a TAMP message with the response to that TAMP message. When the TAMP message is validated directly using a trust anchor, the sequence number is also used to detect TAMP message replay.

To provide replay protection, each TAMP message originator MUST treat the sequence number as a monotonically increasing non-negative integer. The sequence number counter is associated with the signing operation performed by the private key. The trust anchor store MUST ensure that a newly received TAMP message that is validated directly by a trust anchor public key contains a sequence number that is greater than the most recent successfully processed TAMP message from that originator. Note that the sequence Number Adjust message is considered valid if the sequence number is greater than or equal to the most recent successfully processed TAMP message from that 
originator. If the sequence number in a received TAMP message does not meet these conditions, then the trust anchor store MUST reject the TAMP message, returning a sequence number failure (seqNumFailure) error.

Whenever a trust anchor is authorized for TAMP messages, either as a newly installed trust anchor or as a modification to an existing trust anchor, if a sequence number value is not provided in the Trust Anchor Update message, memory MUST be allocated for the sequence number and set to zero. The first TAMP message received that is validated using that trust anchor is not rejected based on sequence number checks, and the sequence number from that first TAMP message is stored. The TAMP message recipient MUST maintain a database of the most recent sequence number from a successfully processed TAMP message from a trust anchor. The index for this database is the trust anchor public key. This could be the apex trust anchor operational public key or a management trust anchor public key. In the first case, the apex trust anchor operational public key is used directly to validate the TAMP message digital signature. In the second case, a management trust anchor public key is used directly to validate the TAMP message digital signature.

Sequence number values MUST be 64-bit non-negative integers. Since ASN.1 encoding of an INTEGER always includes a sign bit, a TAMP message signer can generate 9,223,372,036,854,775,807 TAMP messages before exhausting the 64-bit sequence number space, before which the TAMP message signer MUST transition to a different public/private key pair. The ability to reset a sequence number provided by the Trust Anchor Update and Sequence Number Adjust messages is not intended to avoid the transition to a different key pair; rather, it is intended to aid recovery from operational errors. A relatively small nonvolatile storage requirement is imposed on the trust anchor store for the apex trust anchor and each management trust anchor authorized for TAMP messages.

When the apex trust anchor or a management trust anchor is replaced or removed from the trust anchor store, the associated sequence number storage SHOULD be reclaimed.

7. Subordination Processing

When a TAMP update message is processed, several checks are performed:

- TAMP message authentication is checked including, if necessary, building and validating a certification path to the signer. 
- The signer's authorization is checked, including authorization to manage trust anchors included in the update message.

- Calculation of the trust anchor information to be stored.

This section describes how to perform the second and third steps. Section 1.2 discusses authentication of TAMP messages. Where a trust anchor is represented as a certificate and the calculation of the trust anchor information to be stored is different than the information in the certificate, the TAMP update fails. The TAMP message signer may then wrap the certificate inside a TrustAnchorInfo structure to assert the intended information.

The apex trust anchor is unconstrained, which means that subordination checking need not be performed on Trust Anchor Update messages signed with the apex trust anchor operational public key and that trust anchor information can be stored as it appears in the update message. Subordination checking is performed as part of the validation process of all other Trust Anchor Update messages.

For a Trust Anchor Update message that is not signed with the apex trust anchor operational public key to be valid, the digital signature MUST be validated using an authorized trust anchor, either directly or via an $\mathrm{x} .509$ certification path originating with the apex trust anchor operational public key or an authorized management trust anchor. The following subordination checks MUST also be performed as part of validation of the update message.

Each Trust Anchor Update message contains one or more individual updates, each of which is used to add, modify, or remove a trust anchor. For each individual update, the constraints of the TAMP message signer MUST be greater than or equal to the constraints of the trust anchor in the update. Specifically, constraints included in the CertPathControls field of a TrustAnchorInfo object (or equivalent extensions in Certificate or TBSCertificate objects) must be checked as described below. [RFC5280] describes how the intersection and union operations referenced below are performed.

o The values of the policy flags stored with a trust anchor as the result of a TAMPUpdate are either true or equal to the value of the policy flags associated with the TAMP message signer, i.e., an update may set a flag to false only if the value associated with the TAMP message signer is false. The policy flags associated with the TAMP message signer are read from the policyFlags field or policyConstraints and inhibitAnyPolicy extensions if the signer 
is represented as a trust anchor or from the explicit_policy, policy_mapping, and inhibit_anypolicy state variables following path validation if the signer is not represented as a trust anchor.

- The certificate policies stored with a trust anchor as the result of a TAMPUpdate are equal to the intersection of the value of the certificate policies associated with the TAMP message signer and the value of the policyset field or certificatePolicies extension from the update. The certificate policies associated with the TAMP message signer are read from the policyset field in a TrustAnchorInfo or certificatePolicies extension in a Certificate or TBSCertificate if the signer is represented as a trust anchor or from the valid_policy_tree returned following path validation if the signer is not represented by a trust anchor. Where the TAMP message signer is represented as a trust anchor, no policy mapping is performed. If the intersection is NULL and the to-be-stored requireExplicitPolicy value is true, the TAMP update fails.

- The excluded names stored with a trust anchor as the result of a TAMPUpdate are equal to the union of the excluded names associated with the TAMP message signer and the value from the nameConstr field or nameConstraints extension from the update. The name constraints associated with the TAMP message signer are read from the nameConstr field in a TrustAnchorInfo or nameConstraints extension in a Certificate or TBSCertificate if the signer is a trust anchor or from the excludedSubtrees state variable following path validation if the signer is not a trust anchor. The name of the trust anchor included in the update MUST NOT fall within the excluded name space of the TAMP signer. If the name of the trust anchor falls within the excluded name space of the TAMP signer, the TAMP update fails.

- The permitted names stored with a trust anchor as the result of a TAMPUpdate are equal to the intersection of the permitted names associated with the TAMP message signer and the value from the nameConstr field or nameConstraints extension from the update. The name constraints associated with the TAMP message signer are read from the nameConstr field in a TrustAnchorInfo or nameConstraints extension in a Certificate or TBSCertificate if the signer is a trust anchor or from the permittedSubtrees state variable following path validation if the signer is not a trust anchor. The name of the trust anchor included in the update MUST fall within the permitted name space of the TAMP signer. If the name of the trust anchor does not fall within the permitted name space of the TAMP signer, the TAMP update fails. If the intersection is NULL for all name forms, the TAMP update fails. 
No other extensions defined in [RFC5280] must be processed as part of subordination processing. Other extensions may define subordination rules.

\section{Implementation Considerations}

A public key identifier is used to identify a TAMP message signer. Since there is no guarantee that the same public key identifier is not associated with more than one public key, implementations MUST be prepared for one or more trust anchors to have the same public key identifier. In practical terms, this means that when a digital signature validation fails, the implementation MUST see if there is another trust anchor with the same public key identifier that can be used to validate the digital signature. While duplicate public key identifiers are expected to be rare, implementations MUST NOT fail to find the correct trust anchor when they do occur.

An X.500 distinguished name is used to identify certificate issuers and certificate subjects. The same X.500 distinguished name can be associated with more than one trust anchor. However, the trust anchor public key will be different. The probability that two trust anchors will have the same X.500 distinguished name and the same public key identifier but a different public key is diminishingly small. Therefore, the authority key identifier certificate extension can be used to resolve X.500 distinguished name collisions.

TAMP assumes a reliable underlying transport protocol.

9. Wrapped Apex Contingency Key Certificate Extension

An apex trust anchor MAY contain contingency key information using the WrappedApexContingencykey extension. The extension uses the ApexContingencykey structure as defined below.

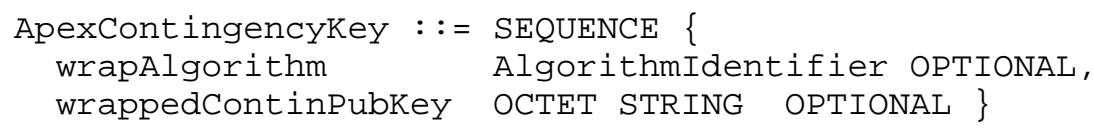

The fields of ApexContingencykey are used as described below. When one field is present, both MUST be present. When one field is absent, both MUST be absent. The fields are allowed to be absent to enable usage of this extension as a means of indicating that the corresponding public key is recognized as an apex trust anchor by some relying parties.

- wrapAlgorithm identifies the symmetric algorithm used to encrypt the apex trust anchor contingency public key. If this public key is ever needed, the symmetric key needed to decrypt it will be 
provided in the message that is to be validated using it. The algorithm identifier is an AlgorithmIdentifier, which contains an object identifier and OPTIONAL parameters. The object identifier indicates the syntax of the parameters, if present.

- wrappedContinPubkey is the encrypted apex trust anchor contingency public key. Once decrypted, it yields the PublickeyInfo structure, which consists of the algorithm identifier followed by the public key itself. The algorithm identifier is an AlgorithmIdentifier that contains an object identifier and OPTIONAL parameters. The object identifier indicates the format of the public key and the syntax of the parameters, if present. The public key is encoded as a BIT STRING.

The WrappedApexContingencykey certificate extension MAY be critical, and it MUST appear at most one time in a set of extensions. The apex trust anchor info extension is identified by the id-pe-wrappedApexContinkey object identifier:

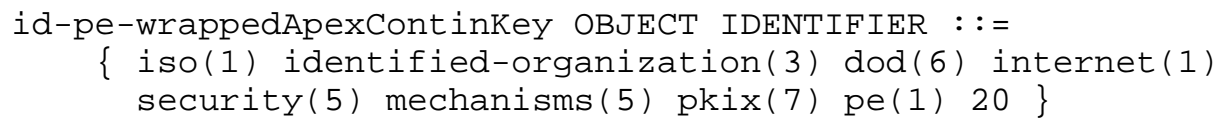

10. Security Considerations

The majority of this specification is devoted to the syntax and semantics of TAMP messages. It relies on other specifications, especially [RFC5914], [RFC3852], and [RFC5280], for the syntax and semantics of trust anchors, intermediate CMS content types, and X.509 certificates, respectively. Since TAMP messages that change the trust anchor state of a trust anchor store are always signed by a Trust Anchor Manager, no further data integrity or data origin authentication mechanisms are needed; however, no confidentiality for these messages is provided. Similarly, certificates are digitally signed, and no additional data integrity or data origin authentication mechanisms are needed. Trust anchor configurations, Trust Anchor Manager certificates, and trust anchor store certificates are not intended to be sensitive. As a result, this specification does not provide for confidentiality of TAMP messages.

Security factors outside the scope of this specification greatly affect the assurance provided. The procedures used by certification authorities (CAs) to validate the binding of the subject identity to their public key greatly affect the assurance associated with the resulting certificate. This is particularly important when issuing certificates to other CAs. In the context of TAMP, the issuance of an end entity certificate under a management trust anchor is an act of delegation. However, such end entities cannot further delegate. 
On the other hand, issuance of a CA certificate under a management trust anchor is an act of delegation where the CA can perform further delegation. The scope of the delegation can be constrained by including appropriate certificate extensions in a CA certificate.

X.509 certification path construction involves comparison of X.500 distinguished names. Inconsistent application of name comparison rules can result in acceptance of invalid X.509 certification paths or rejection of valid ones. Name comparison can be extremely complex. To avoid imposing this complexity on trust anchor stores, any certificate profile used with TAMP SHOULD employ simple name structures and impose rigorous restrictions on acceptable distinguished names, including the way that they are encoded. The goal of that certificate profile should be to enable simple binary comparison. That is, case conversion, character set conversion, white space compression, and leading and trailing white space trimming SHOULD be avoided.

Some digital signature algorithms (DSAs) require the generation of random one-time values. For example, when generating a DSA digital signature, the signer MUST generate a random k value [DSS]. Also, the generation of public/private key pairs relies on random numbers.

The use of an inadequate random number generator (RNG) or an inadequate pseudo-random number generator (PRNG) to generate such cryptographic values can result in little or no security. An attacker may find it much easier to reproduce the random number generation environment, searching the resulting small set of possibilities, rather than brute-force searching the whole space.

Compromise of an identity trust anchor private key permits unauthorized parties to issue certificates that will be acceptable to all trust anchor stores configured with the corresponding identity trust anchor. The unauthorized private key holder will be limited by the certification path controls associated with the identity trust anchor. For example, clearance constraints in the identity trust anchor will determine the clearances that will be accepted in certificates that are issued by the unauthorized private key holder.

Compromise of a management trust anchor private key permits unauthorized parties to generate signed messages that will be acceptable to all trust anchor stores configured with the corresponding management trust anchor. All devices that include the compromised management trust anchor can be configured as desired by the unauthorized private key holder within the limits of the subordination checks described in section 7. If the management trust anchor is associated with content types other than TAMP, then the unauthorized private key holder can generate signed messages of that 
type. For example, if the management trust anchor is associated with firmware packages, then the unauthorized private key holder can install different firmware.

Compromise of the apex trust anchor operational private key permits unauthorized parties to generate signed messages that will be acceptable to all trust anchor stores configured with the corresponding apex trust anchor. All devices that include that apex trust anchor can be configured as desired by the unauthorized private key holder, and the unauthorized private key holder can generate signed messages of any content type. The optional contingency private key offers a potential way to recover from such a compromise.

The compromise of a $\mathrm{CA}^{\prime}$ s private key leads to the same type of problems as the compromise of an identity or a management trust anchor private key. The unauthorized private key holder will be limited by the certification path controls and extensions associated with the trust anchor.

The compromise of an end entity private key leads to the same type of problems as the compromise of an identity or a management trust anchor private key, except that the end entity is unable to issue any certificates. The unauthorized private key holder will be limited by the certification path controls and extensions associated with the trust anchor.

Compromise of a trust anchor store's digital signature private key permits unauthorized parties to generate signed TAMP response messages, masquerading as the trust anchor store.

Premature disclosure of the key-encryption key used to encrypt the apex trust anchor contingency public key may result in early exposure of the apex trust anchor contingency public key.

TAMP implementations need to be able to parse messages and certificates. Care must be taken to ensure that there are no implementation defects in the TAMP message parser or the processing that acts on the message content. A validation suite is one way to increase confidence in the parsing of TAMP messages, CMS content types, attributes, certificates, and extensions.

TrustAnchorlist messages do not provide a replay detection mechanism. Where TrustAnchorlist messages are accepted as an alternative means of adding trust anchors to a trust anchor store, applications may require additional mechanisms to address the risks associated with replay of old TrustAnchorList messages. 
As sequence number values are used to detect replay attempts, trust anchor store managers must take care to maintain their own sequence number state, i.e., knowledge of which sequence number to include in the next TAMP message generated by the trust anchor store manager. Loss of sequence number state can result in generation of TAMP messages that cannot be processed due to seqNumFailure. In the event of loss, sequence number state can be restored by inspecting the most recently generated TAMP message, provided the messages are logged, or in collaboration with a trust anchor store manager who can successfully issue a TAMPStatusQuery message.

\section{IANA Considerations}

The details of TAMP requests and responses are communicated using object identifiers (OIDs). The objects are defined in an arc delegated by IANA to the PKIX working group. This document also includes eleven media type registrations in Appendix B. No further action by IANA is necessary for this document or any anticipated updates.

12. References

12.1. Normative References

[RFC2119] Bradner, S., "Key words for use in RFCs to Indicate Requirement Levels", BCP 14, RFC 2119, March 1997.

[RFC2616] Fielding, R., Gettys, J., Mogul, J., Frystyk, H., Masinter, L., Leach, P., and T. Berners-Lee, "Hypertext Transfer Protocol -- HTTP/1.1", RFC 2616, June 1999.

[RFC3986] Berners-Lee, T., Fielding, R., and L. Masinter, "Uniform Resource Identifier (URI): Generic Syntax", STD 66, RFC 3986, January 2005.

[RFC5280] Cooper, D., Santesson, S., Farrell, S., Boeyen, S., Housley, R., and W. Polk, "Internet X.509 Public Key Infrastructure Certificate and Certificate Revocation List (CRL) Profile", RFC 5280, May 2008.

[RFC5652] Housley, R., "Cryptographic Message Syntax (CMS)", RFC 5652, september 2009.

[RFC5912] Hoffman, P. and J. Schaad, "New ASN.1 Modules for the Public Key Infrastructure Using X.509 (PKIX)", RFC 5912, June 2010. 
[RFC5914] Housley, R., Ashmore, S., and C. Wallace, "Trust Anchor Format", RFC 5914, June 2010.

$[\mathrm{X} .680]$

"ITU-T Recommendation X.680 - Information Technology

- Abstract Syntax Notation One", 1997.

$[\mathrm{X} .690]$

"ITU-T Recommendation X.690 - Information Technology - ASN.1 encoding rules: Specification of Basic Encoding Rules (BER), Canonical Encoding Rules (CER) and Distinguished Encoding Rules (DER)", 1997.

\subsection{Informative References}

[DSS ]

[PKCS\# 6]

[RFC32 79]

[RFC3370]

[RFC 4049 ]

[RFC 4108 ]

[RFC5753]

[RFC5754]

[RFC5755]

[ TA-MGMT-REQS ]
"FIPS Pub 186: Digital Signature Standard", May 1994.

"PKCS \#6: Extended-Certificate Syntax Standard, Version 1.5", November 1993.

Bassham, L., Polk, W., and R. Housley, "Algorithms and Identifiers for the Internet X.509 Public Key Infrastructure Certificate and Certificate Revocation List (CRL) Profile", RFC 3279, April 2002.

Housley, R., "Cryptographic Message Syntax (CMS) Algorithms", RFC 3370, August 2002 .

Housley, R., "BinaryTime: An Alternate Format for Representing Date and Time in ASN.1", RFC 4049, April 2005 .

Housley, R., "Using Cryptographic Message Syntax (CMS) to Protect Firmware Packages", RFC 4108, August 2005 .

Turner, S. and D. Brown, "Use of Elliptic Curve Cryptography (ECC) Algorithms in Cryptographic Message Syntax (CMS)", RFC 5753, January 2010.

Turner, S., "Using SHA2 Algorithms with Cryptographic Message Syntax", RFC 5754, January 2010.

Farrell, S., Housley, R., and S. Turner, "An Internet Attribute Certificate Profile for Authorization", RFC 5755, January 2010 .

Reddy, R. and C. Wallace, "Trust Anchor Management Requirements", Work in Progress, March 2010. 
$[\mathrm{X} .208]$

"ITU-T Recommendation X.208 - Specification of Abstract Syntax Notation One (ASN.1)", 1988.

$[\mathrm{X} .509]$

"ITU-T Recommendation X.509 - The Directory Authentication Framework", 2000. 
Appendix A. ASN.1 Modules

Appendix A.1 provides the normative ASN.1 definitions for the structures described in this specification using ASN.1 as defined in [X.680]. Appendix A.2 provides a module using ASN.1 as defined in [X.208]. The module in Appendix A.2 removes usage of newer ASN.1 features that provide support for limiting the types of elements that may appear in certain SEQUENCE and SET constructions. Otherwise, the modules are compatible in terms of encoded representation, i.e., the modules are bits-on-the-wire compatible aside from the limitations on SEQUENCE and SET constituents. Extension markers are not used due to lack of support in [X.208]. Appendix A.2 is included as a courtesy to developers using ASN.1 compilers that do not support current ASN.1. Appendix A.1 includes definitions imported from [RFC5280], [RFC5912], and [RFC5914].

A.1. ASN.1 Module Using 1993 Syntax

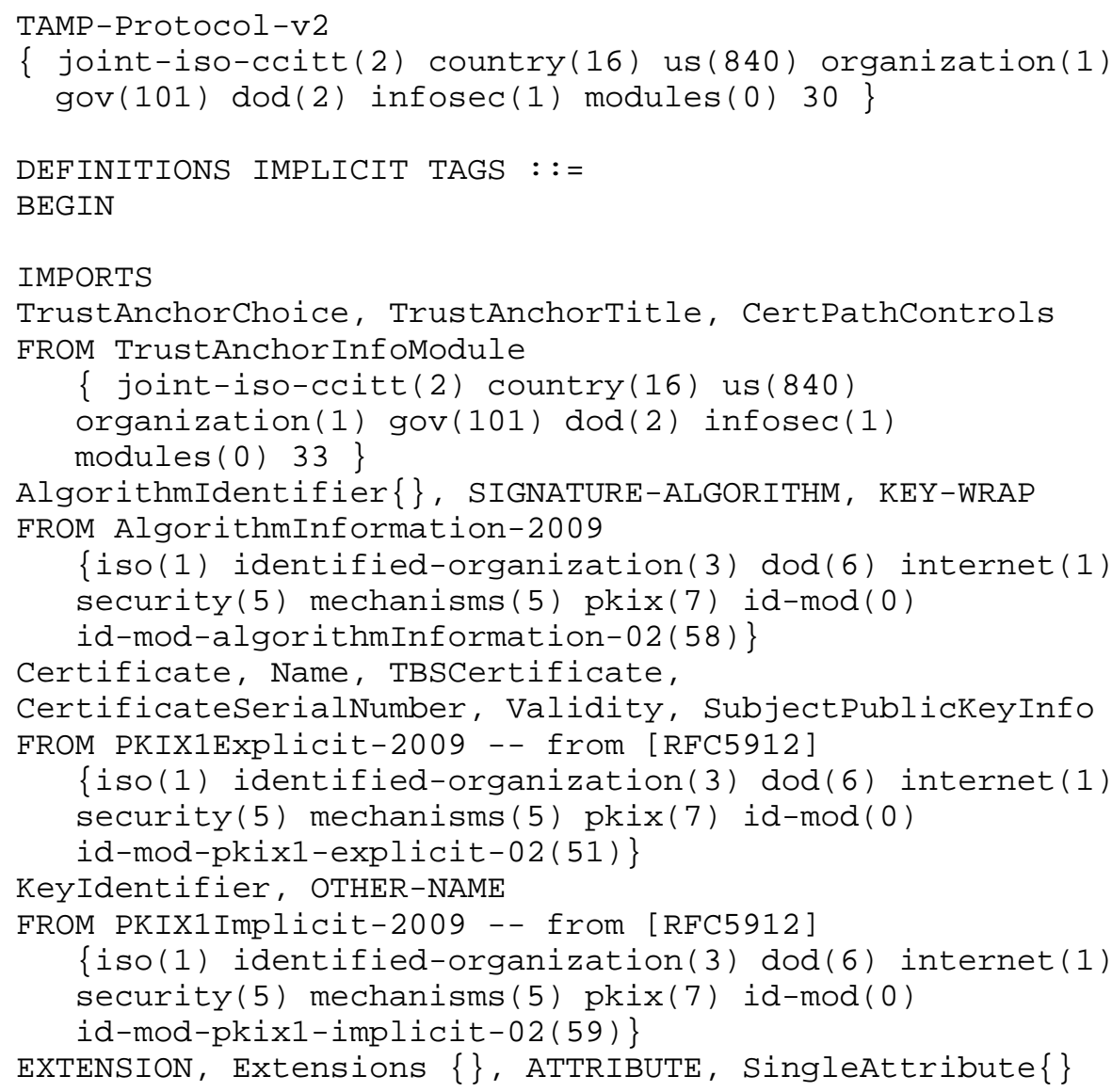




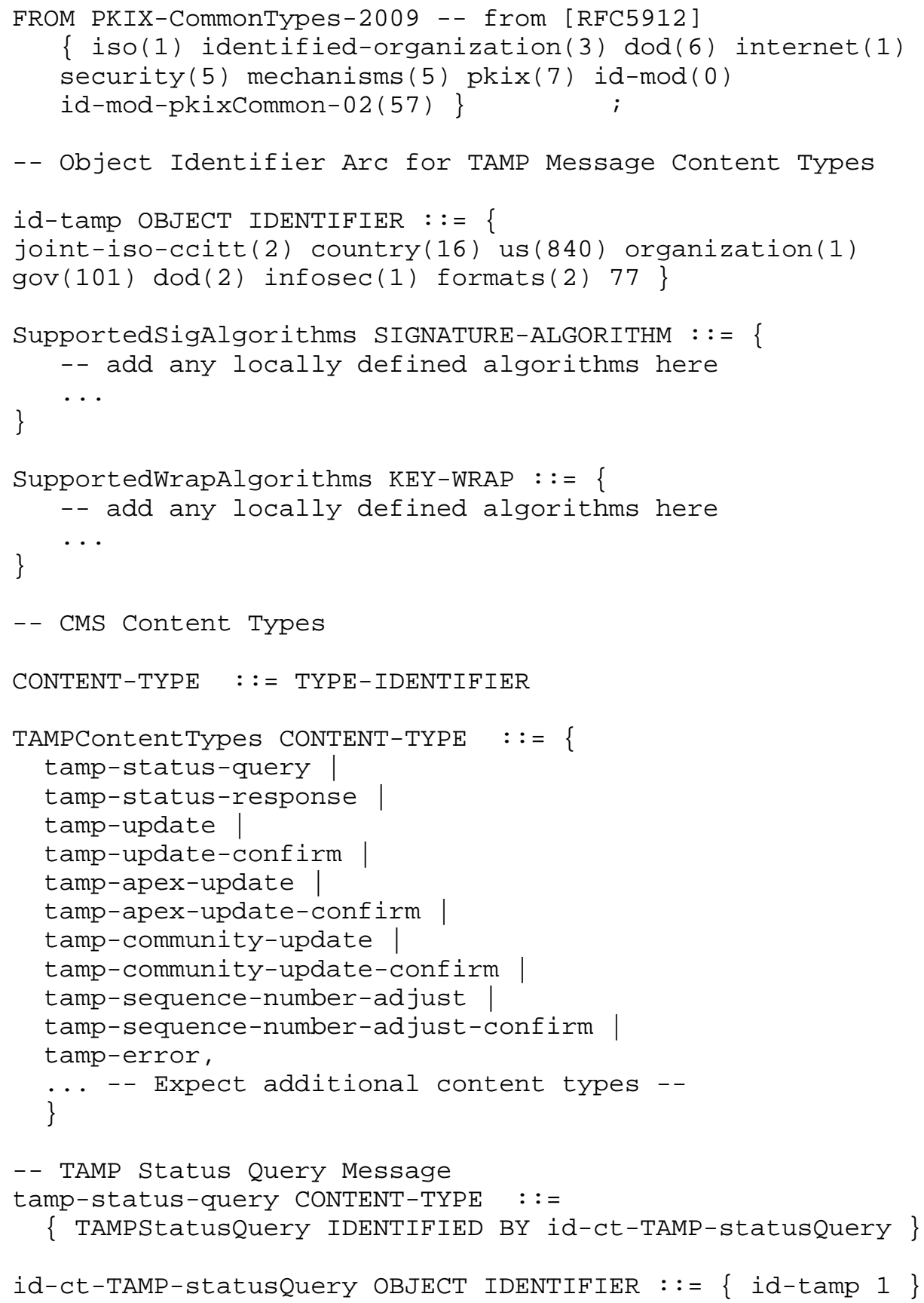




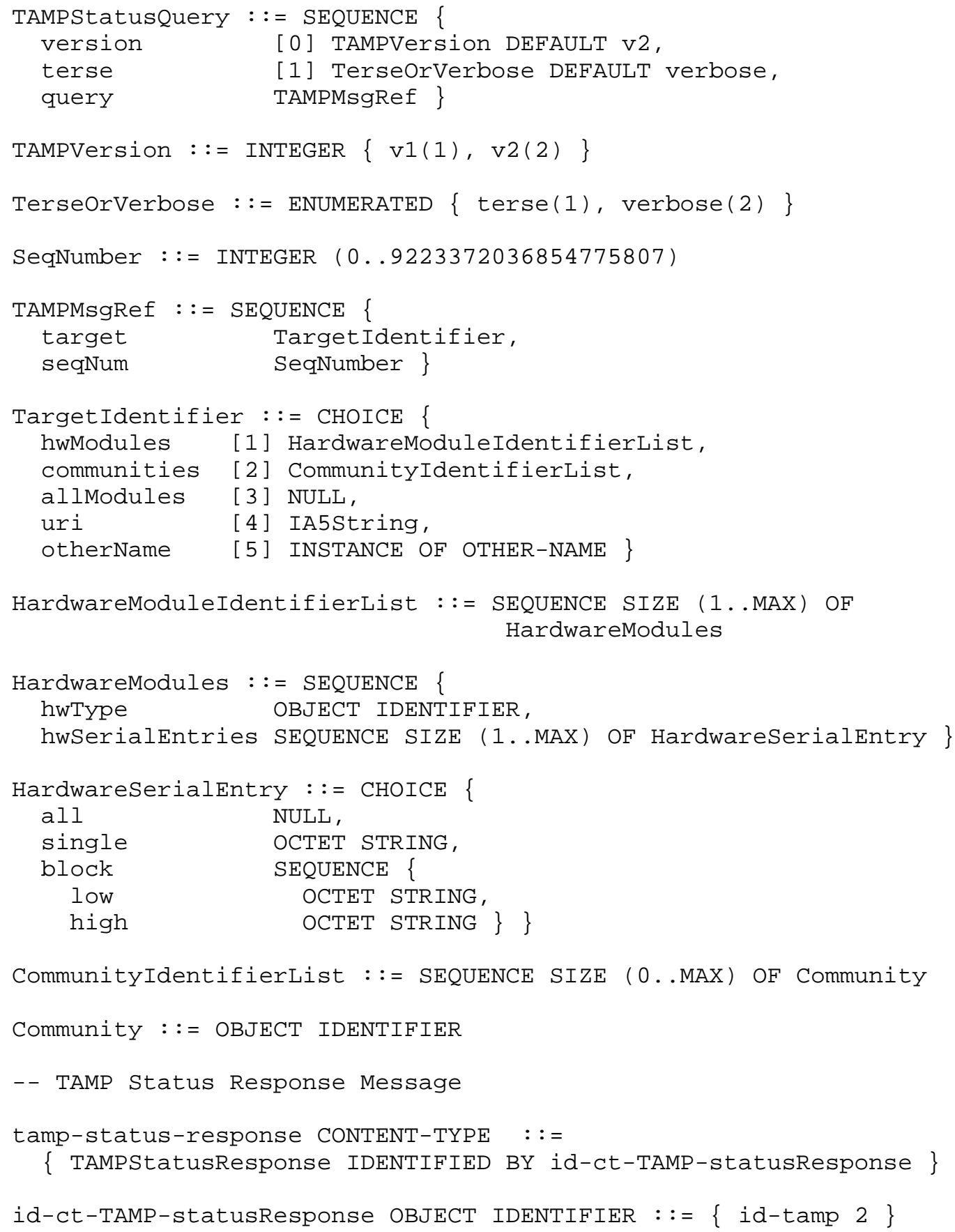




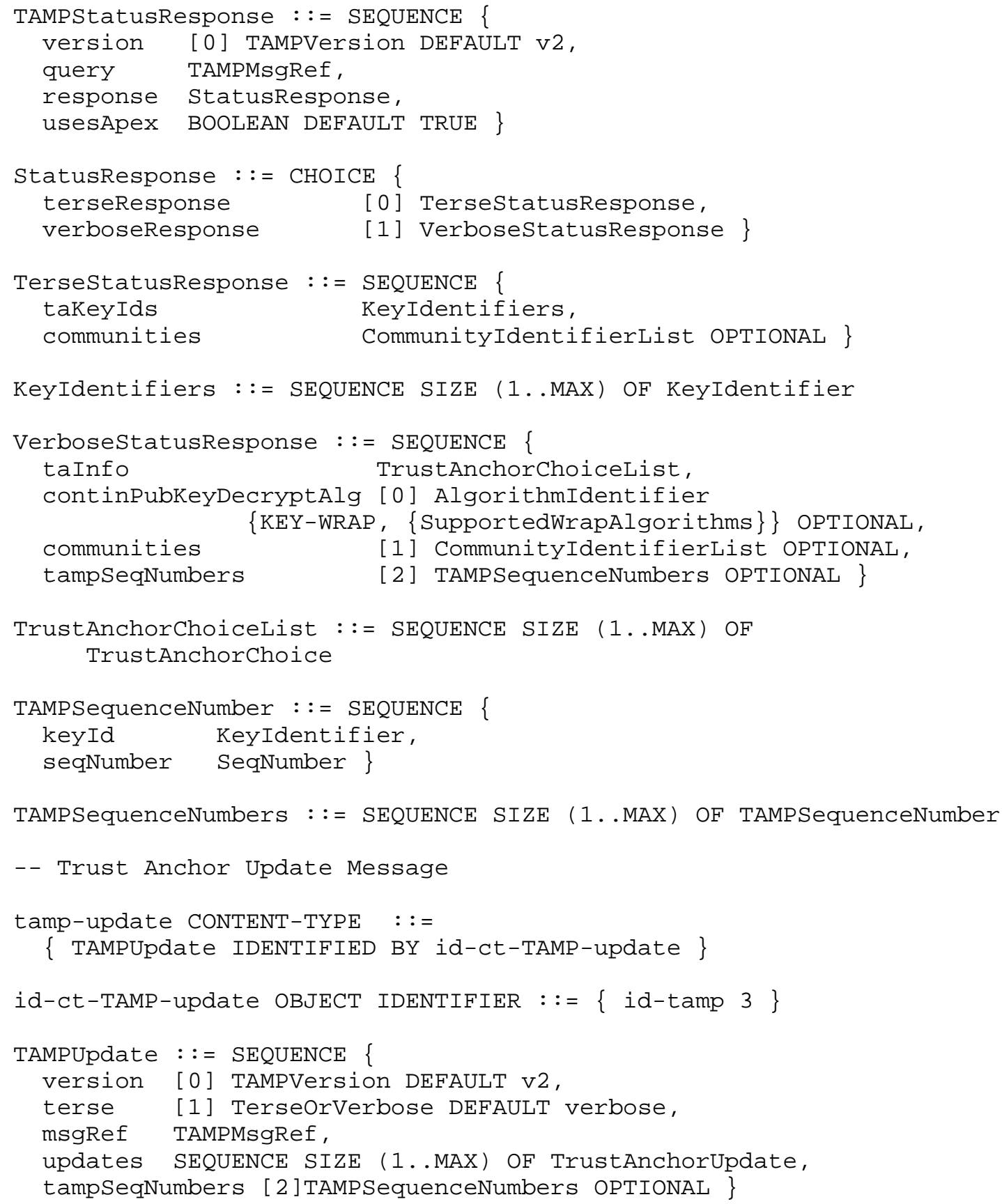




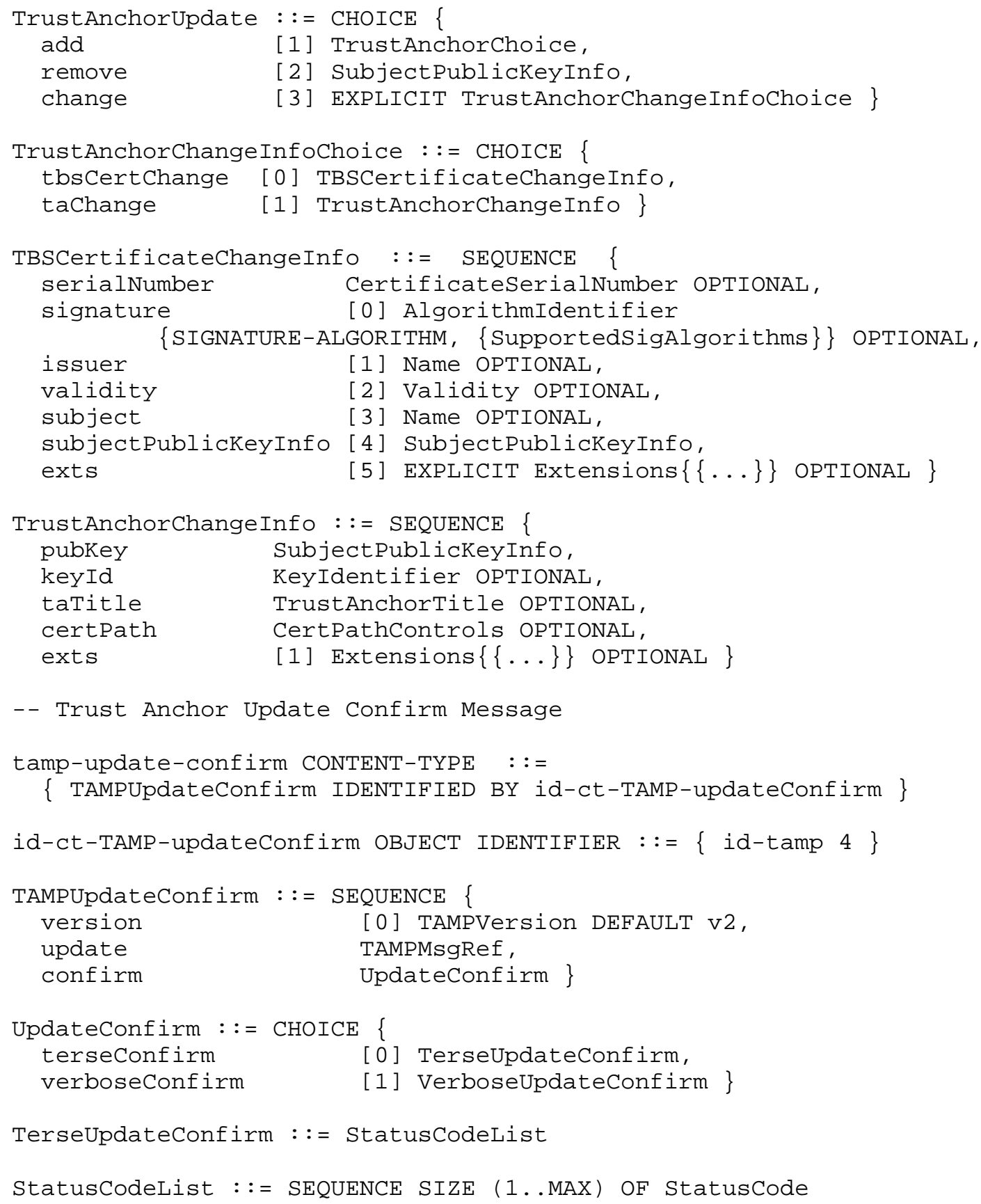




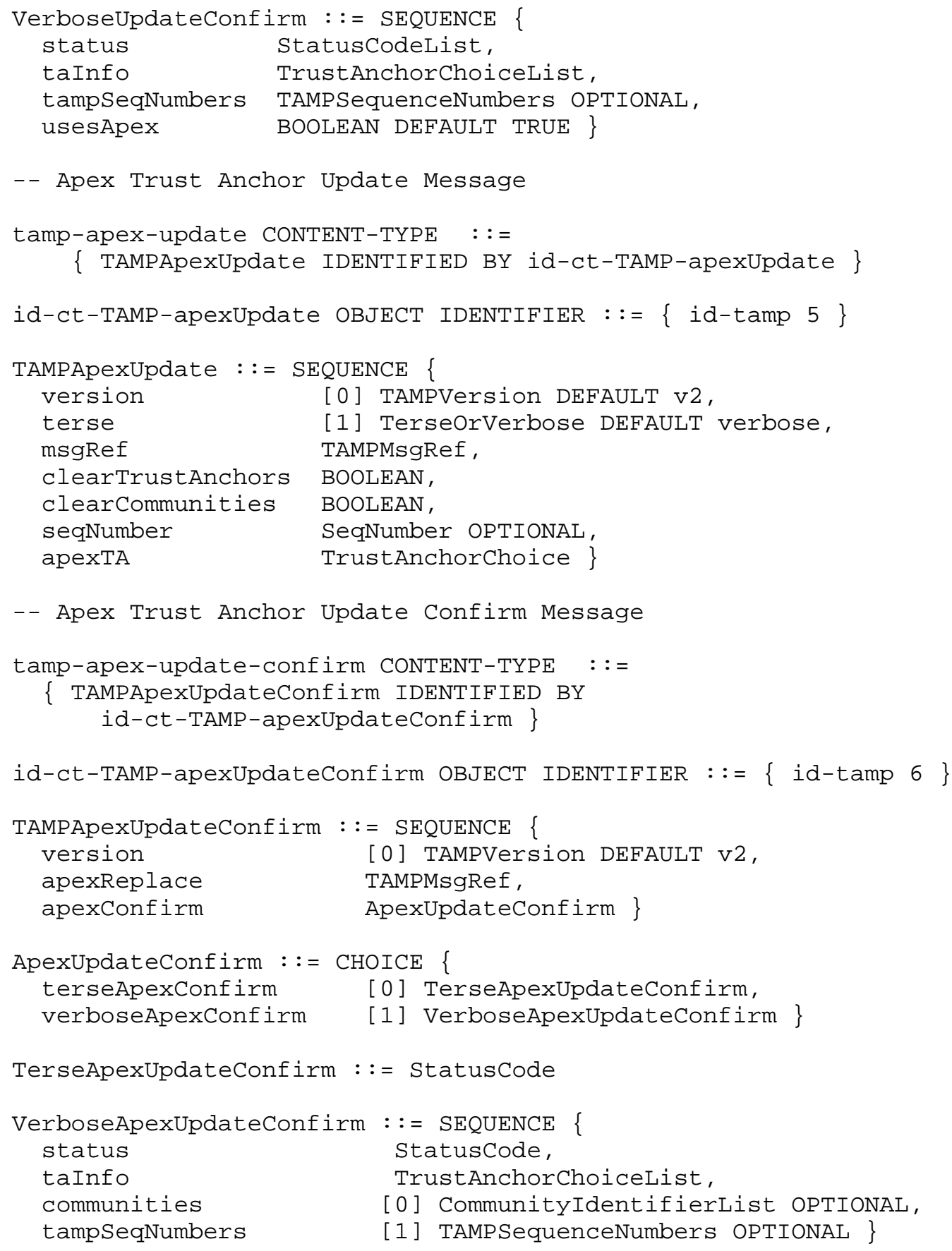




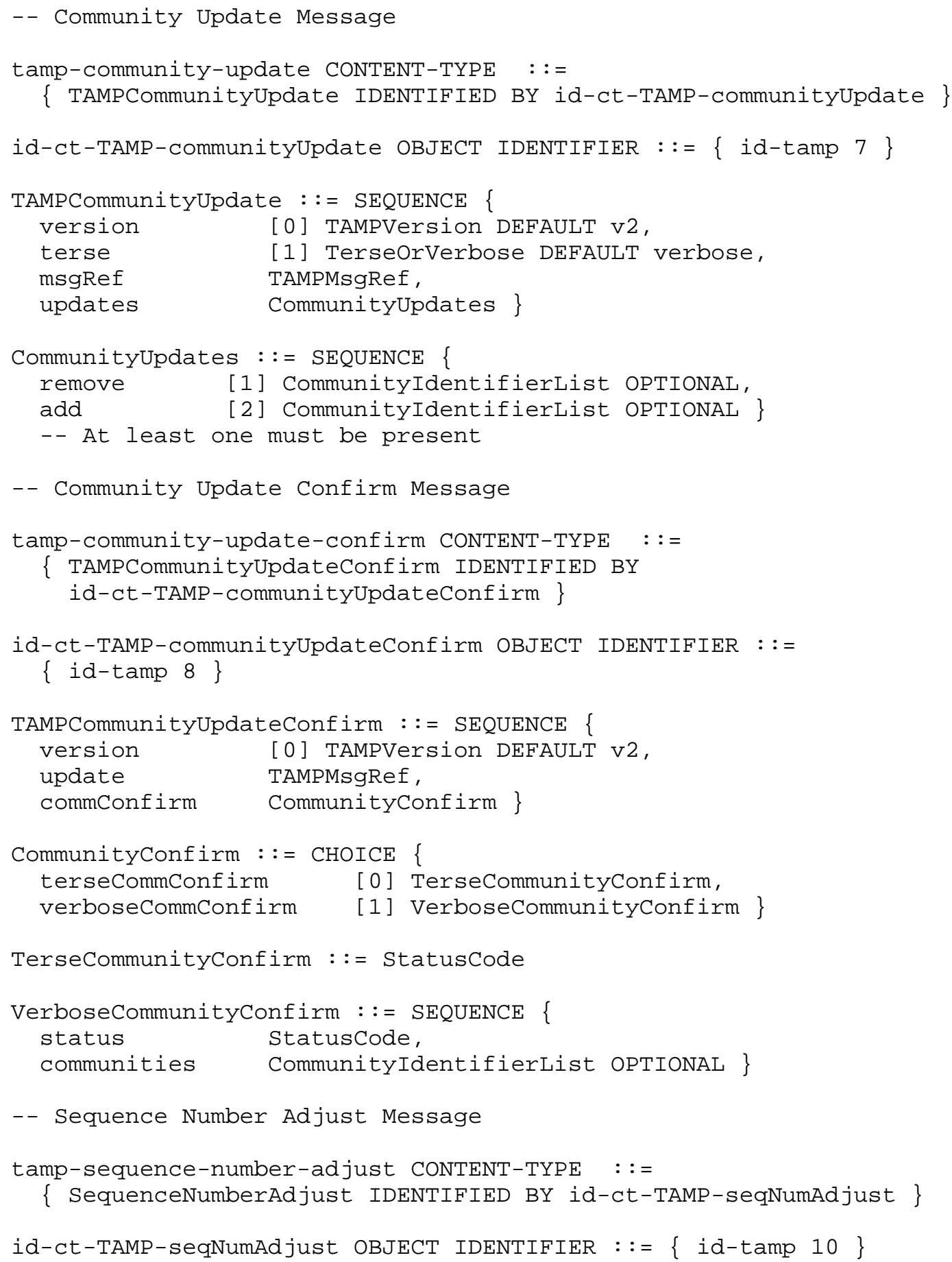




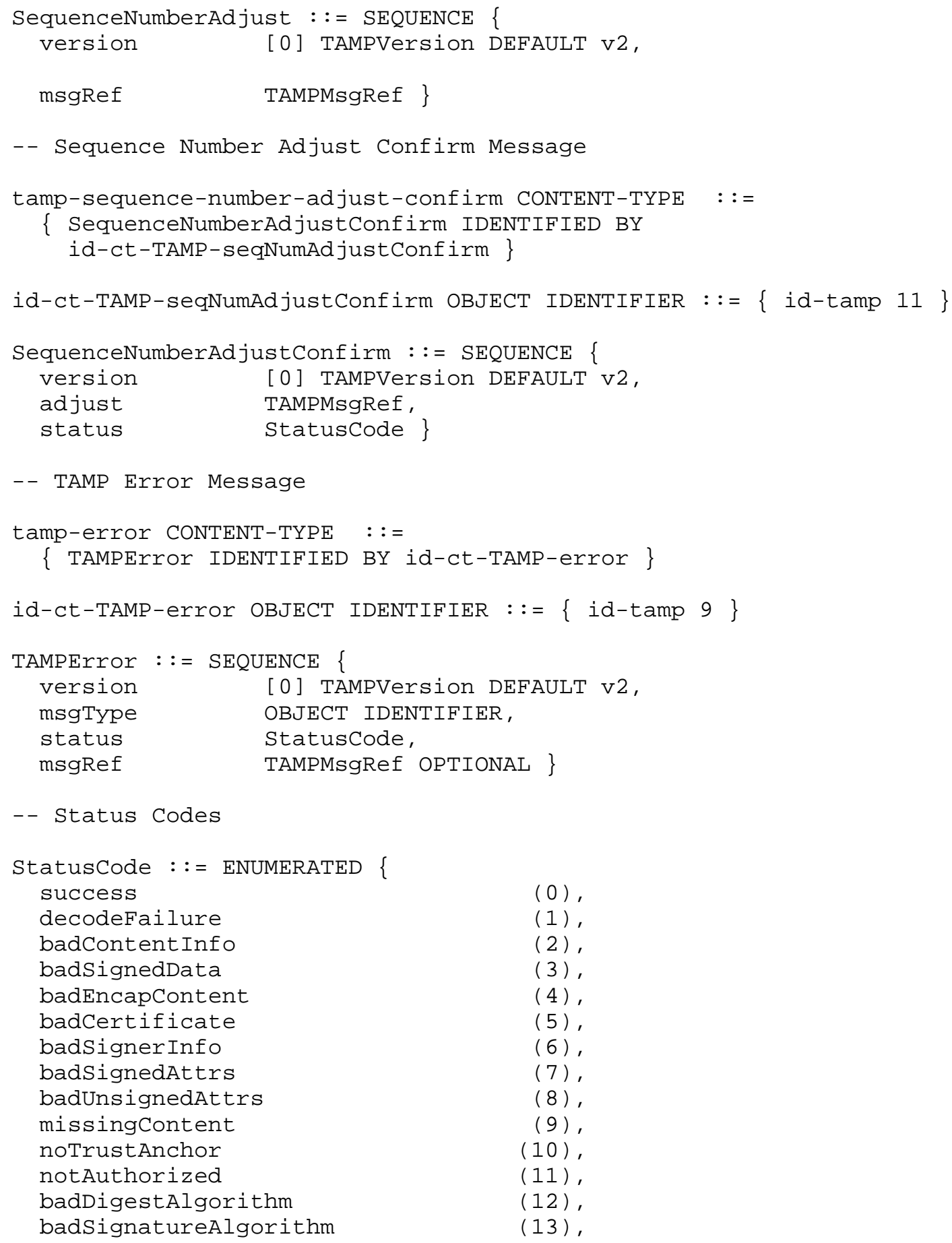




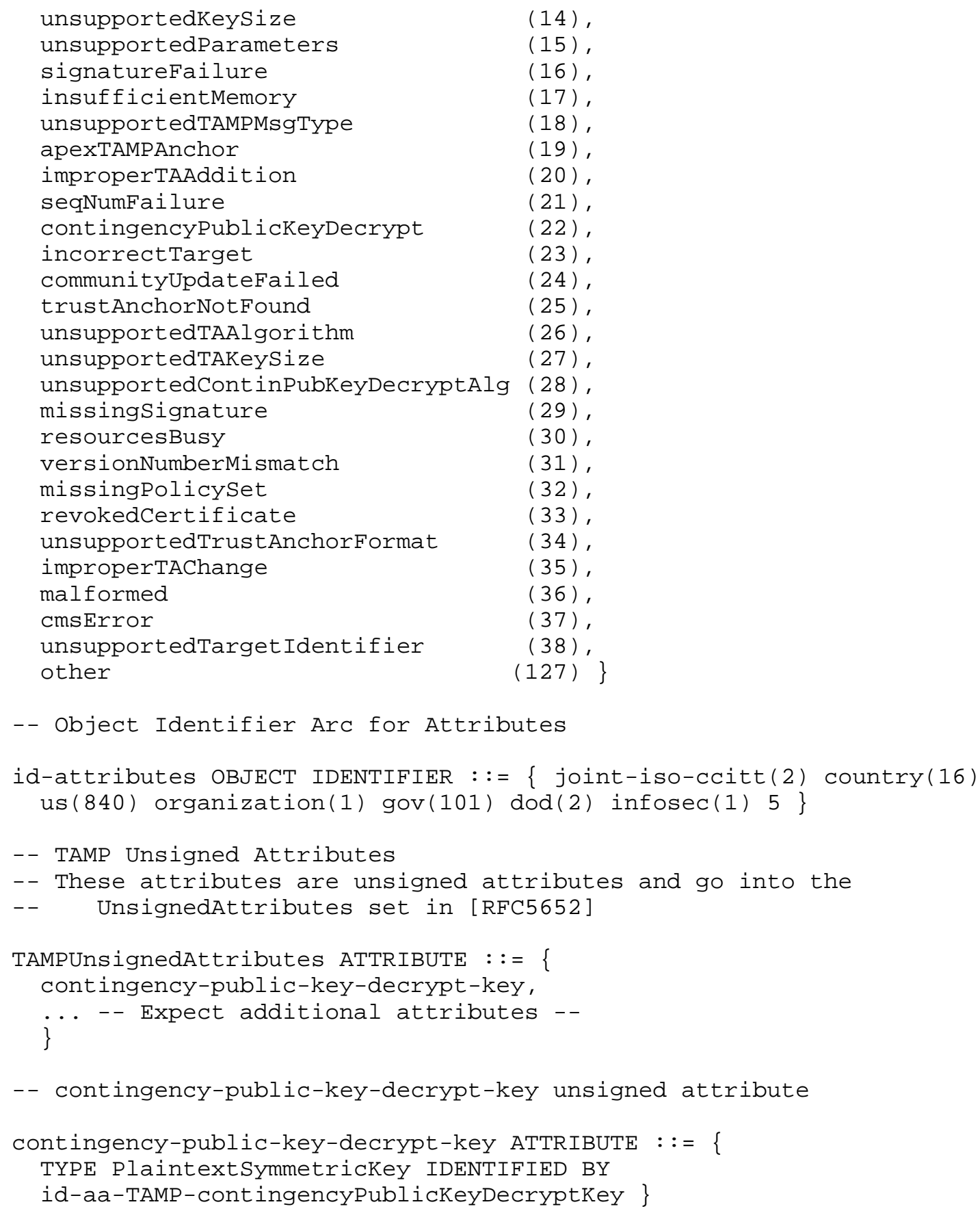




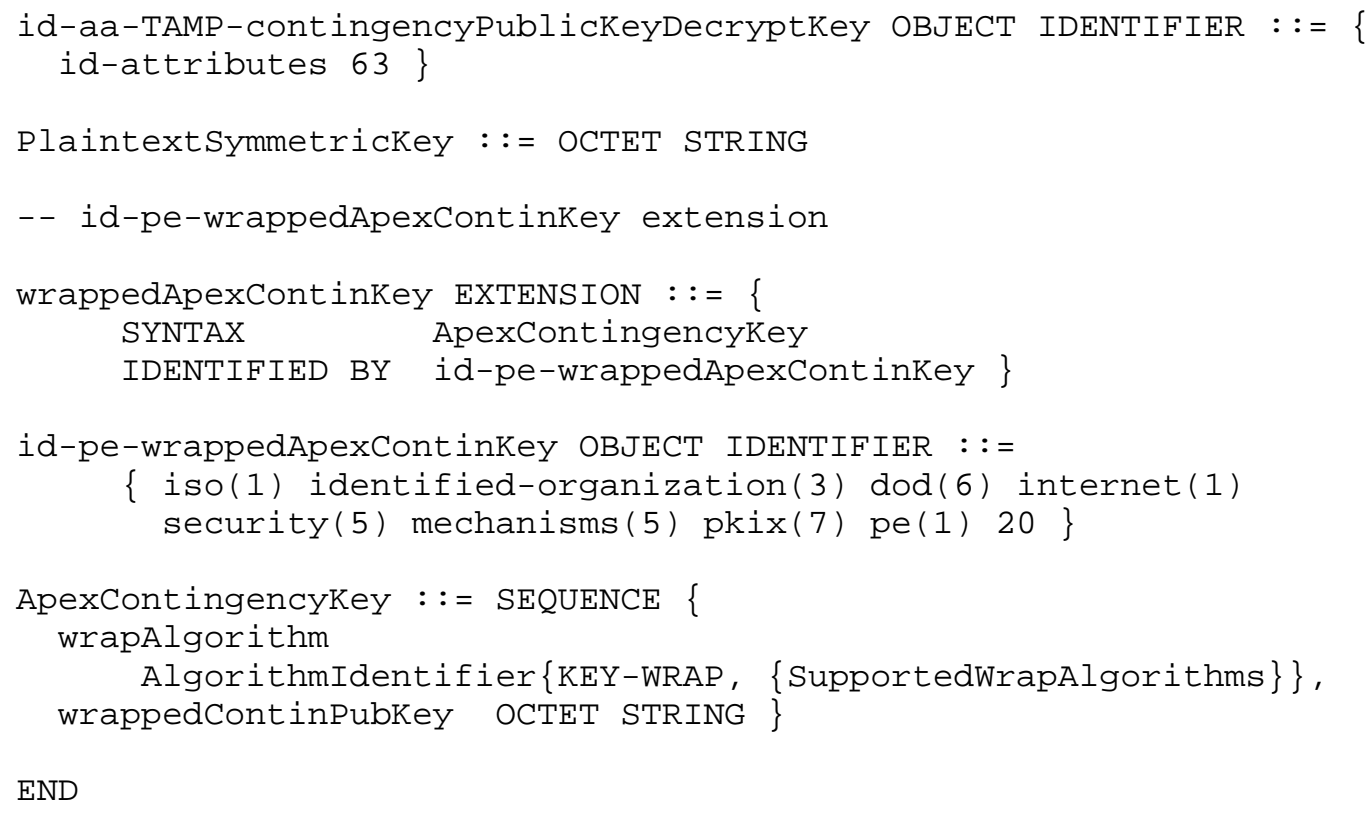




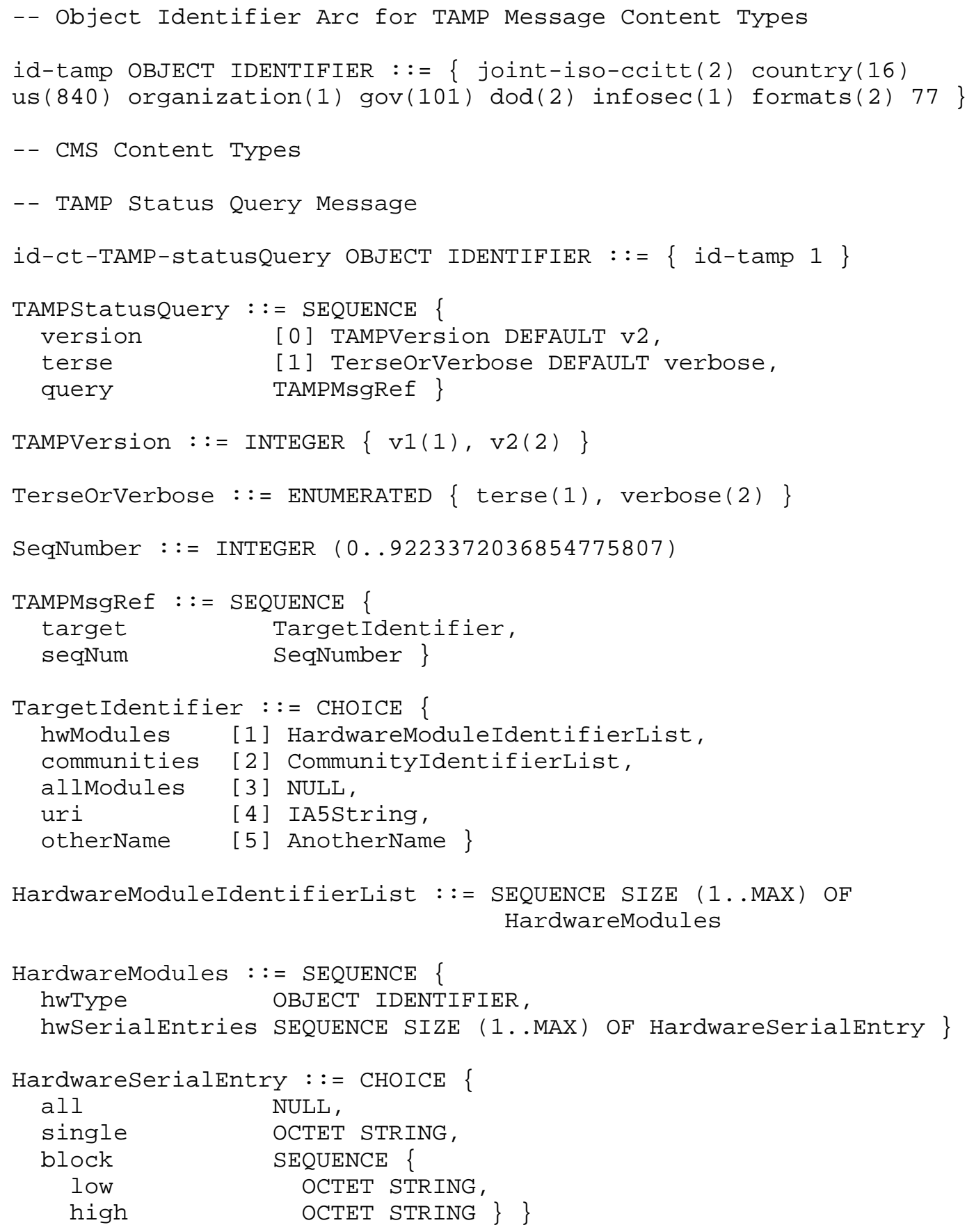




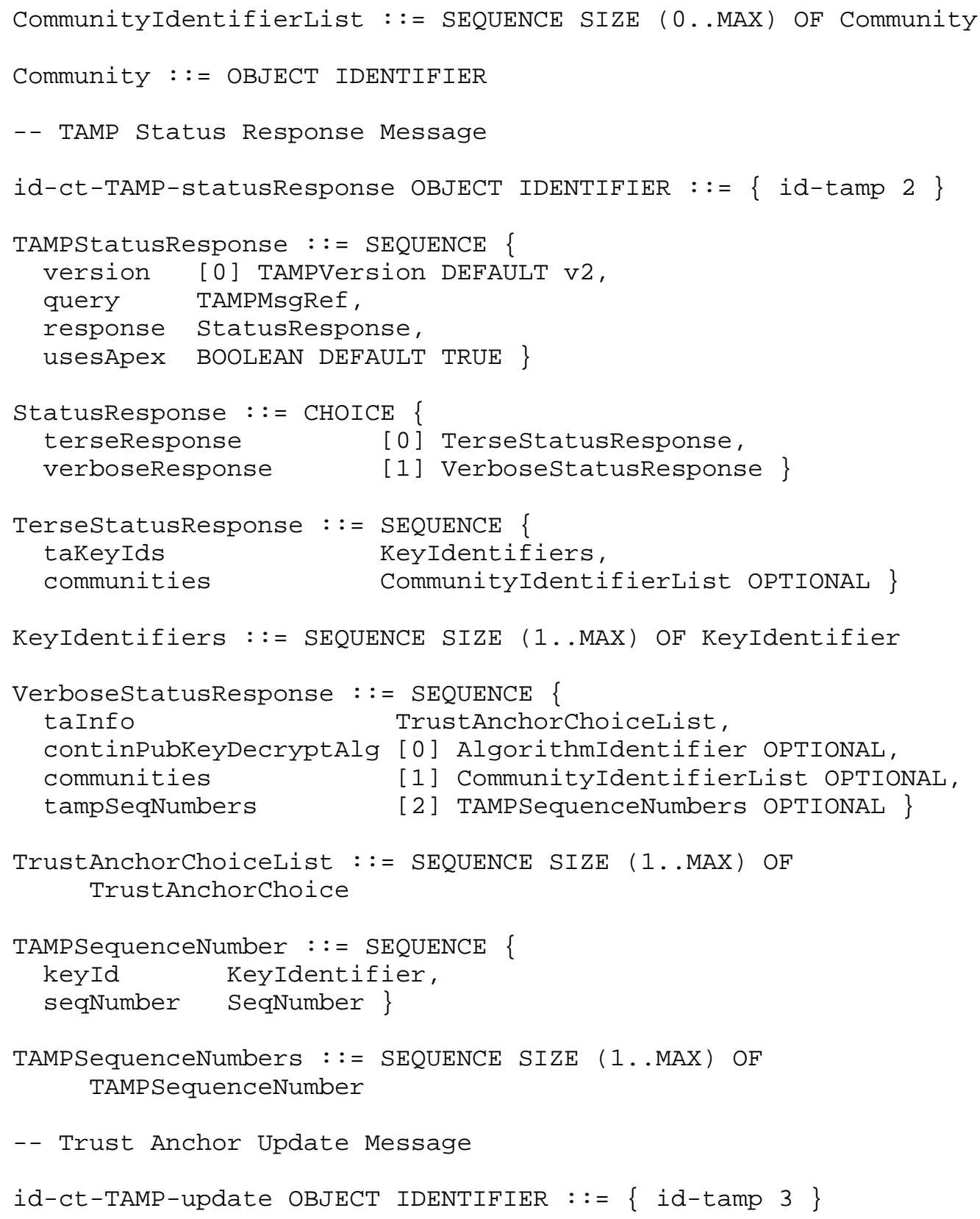




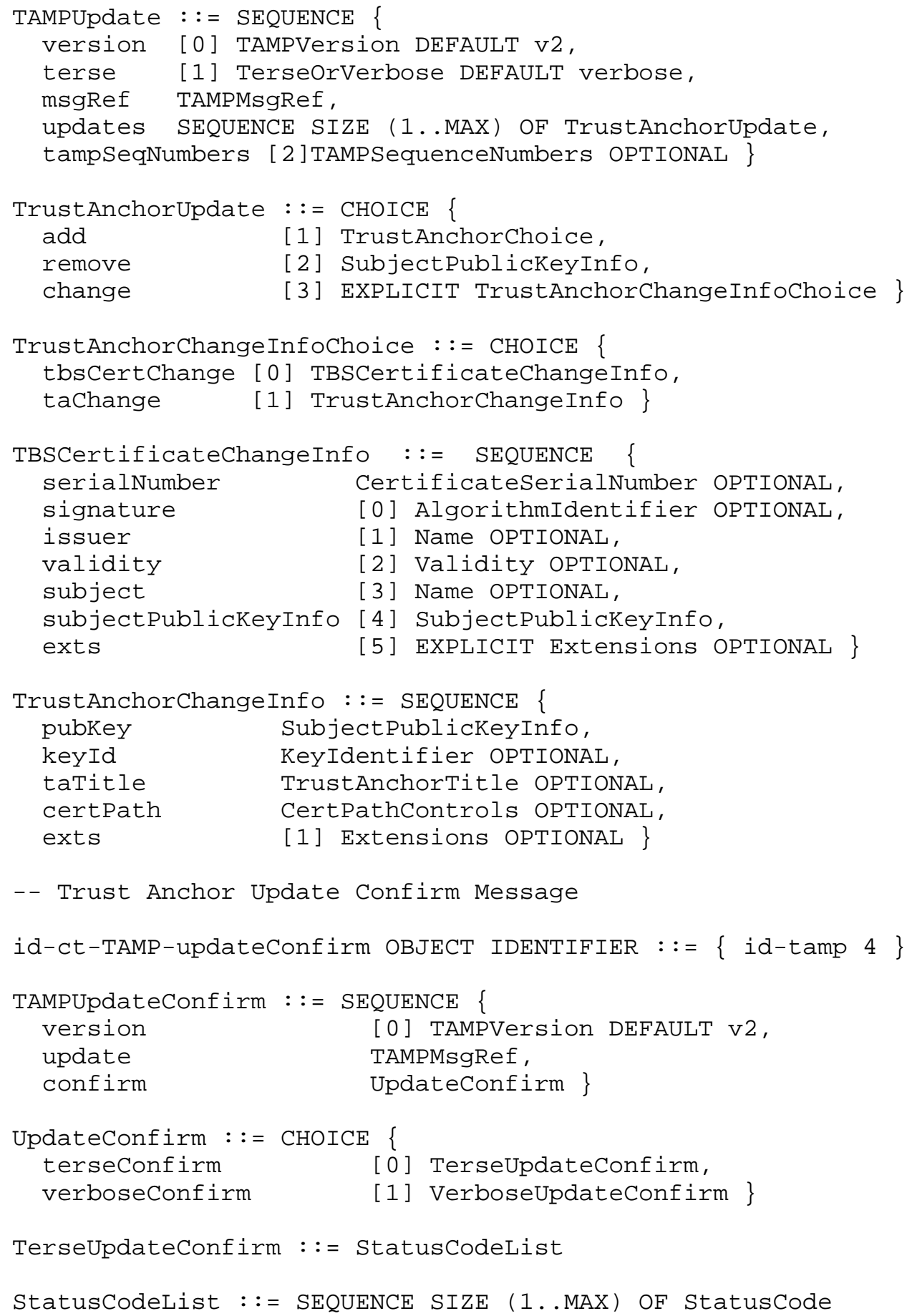




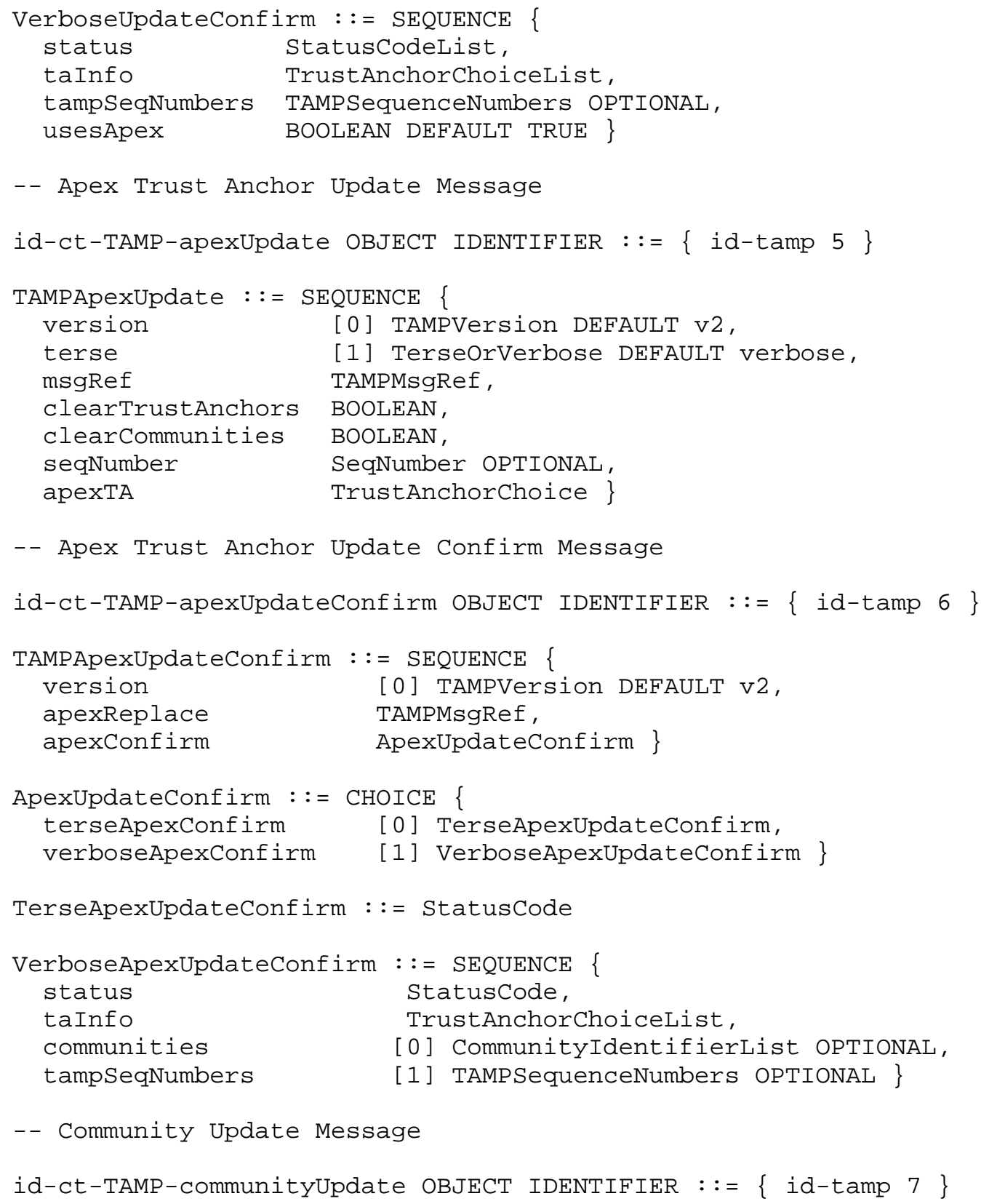




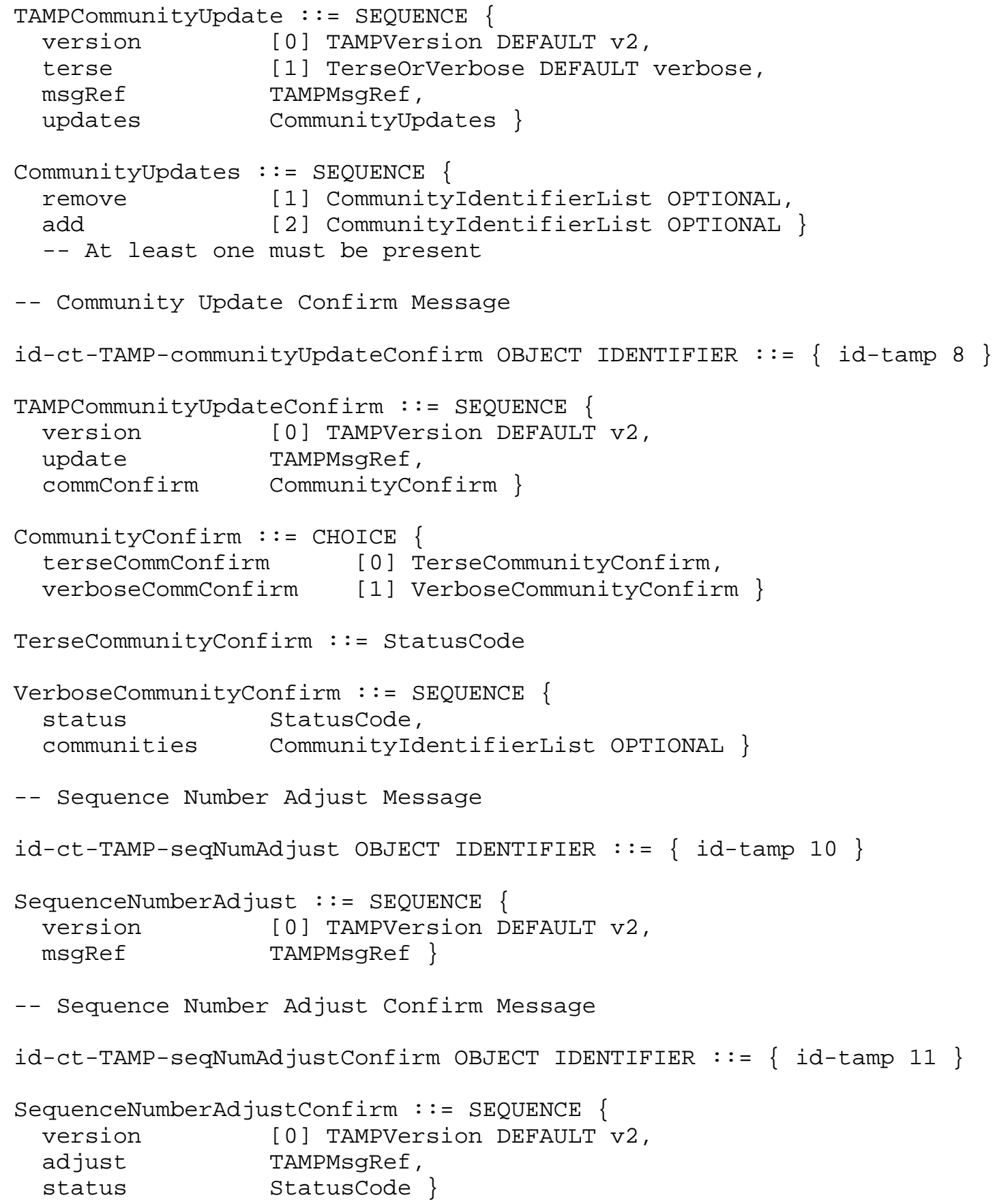




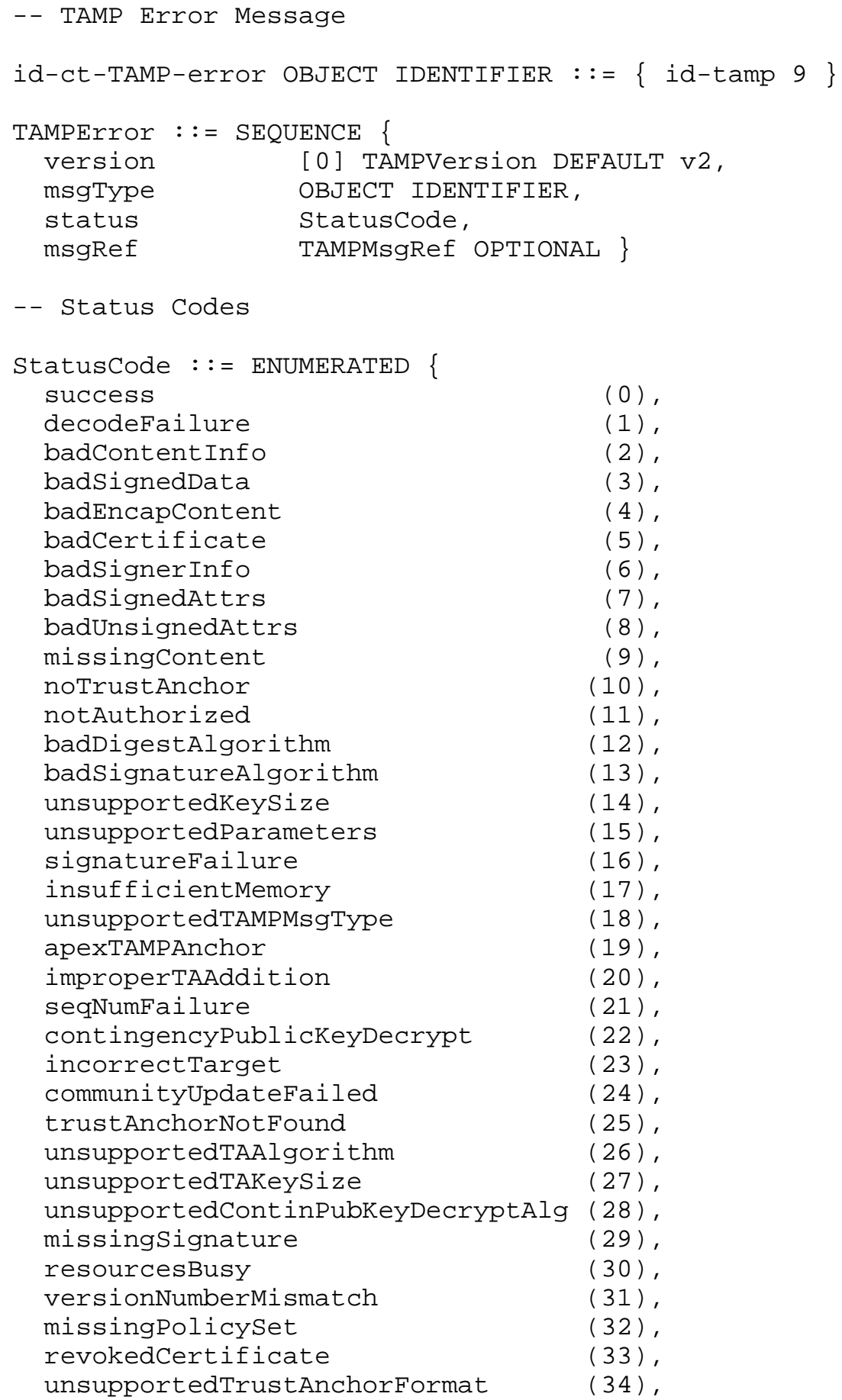




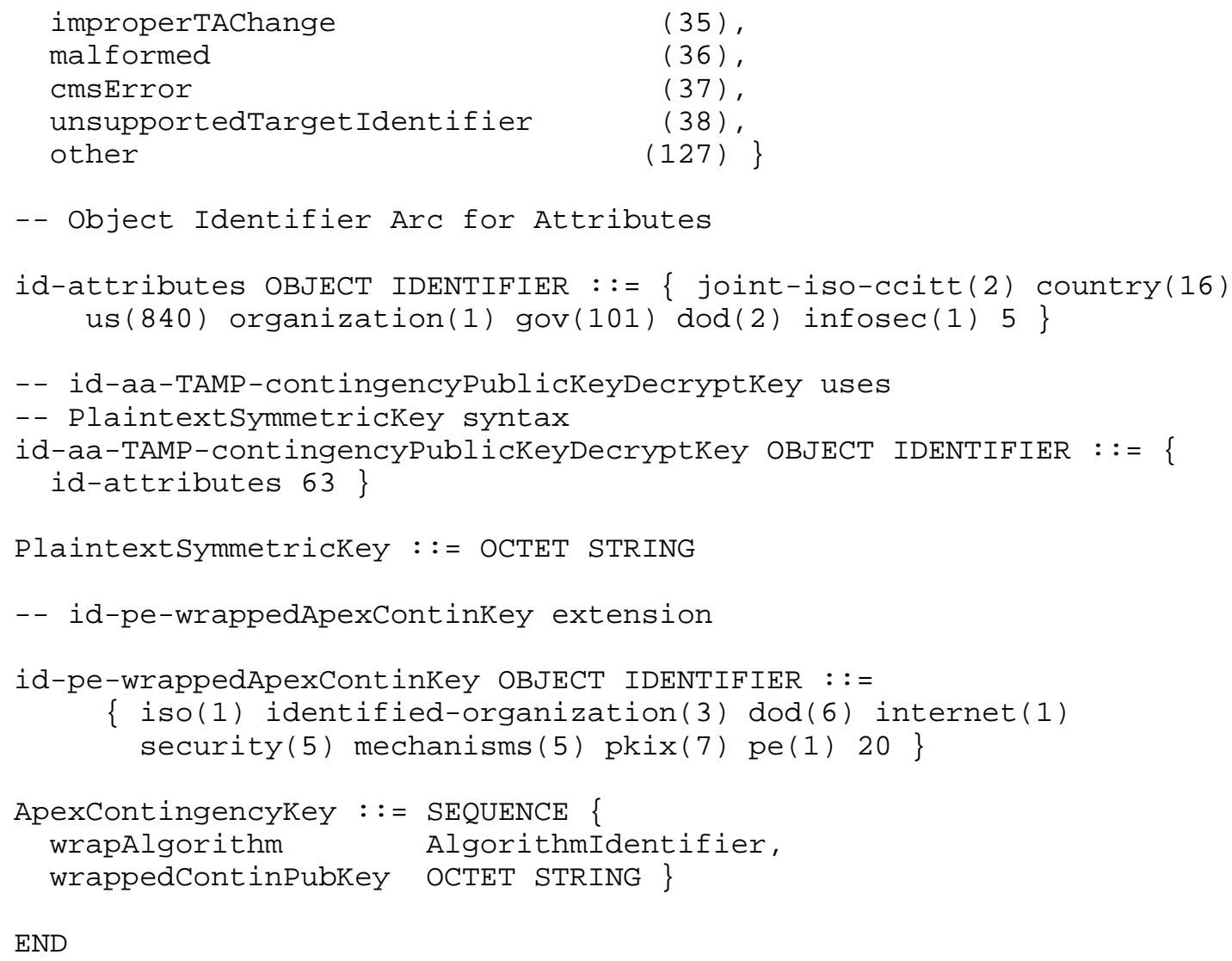




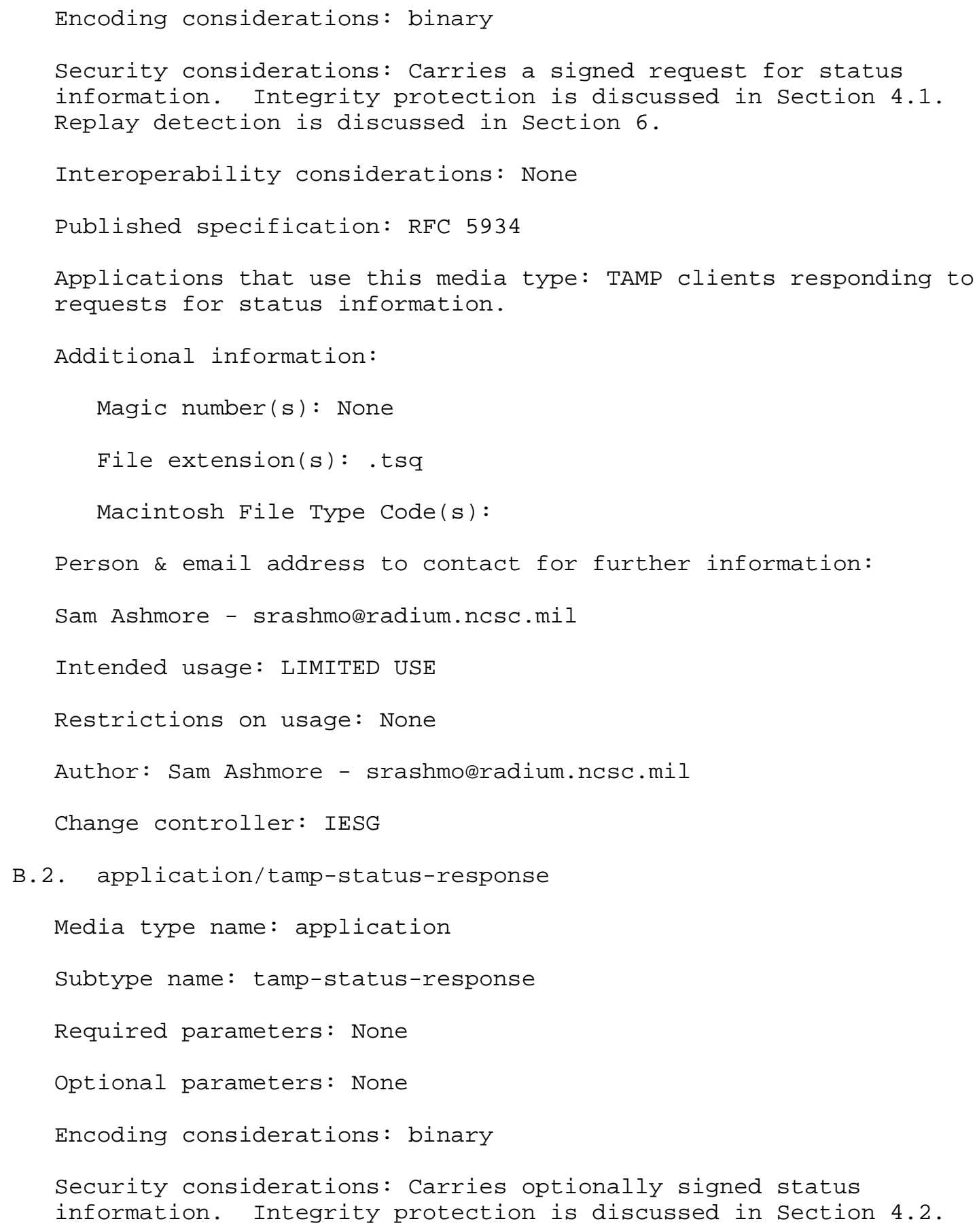




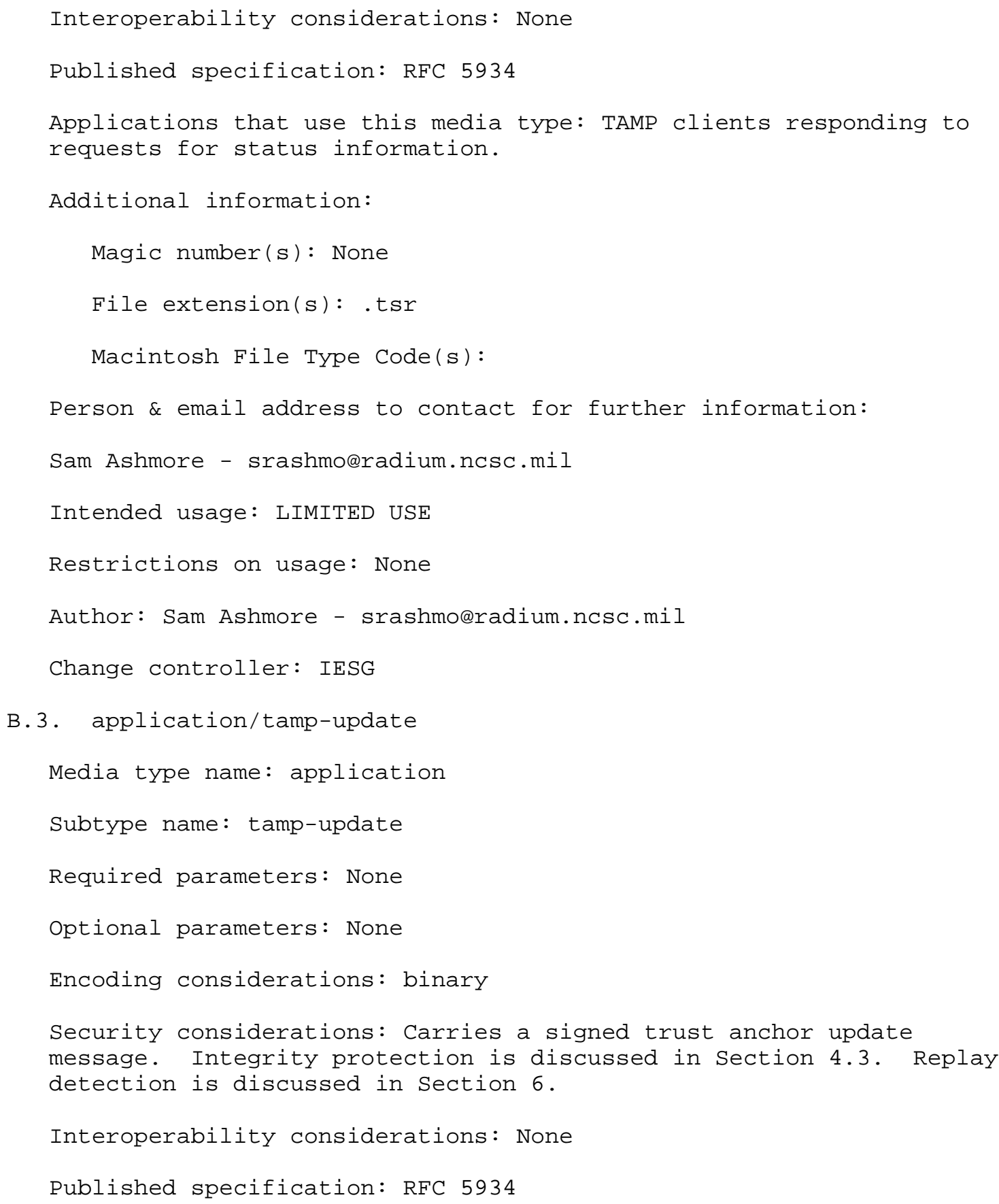




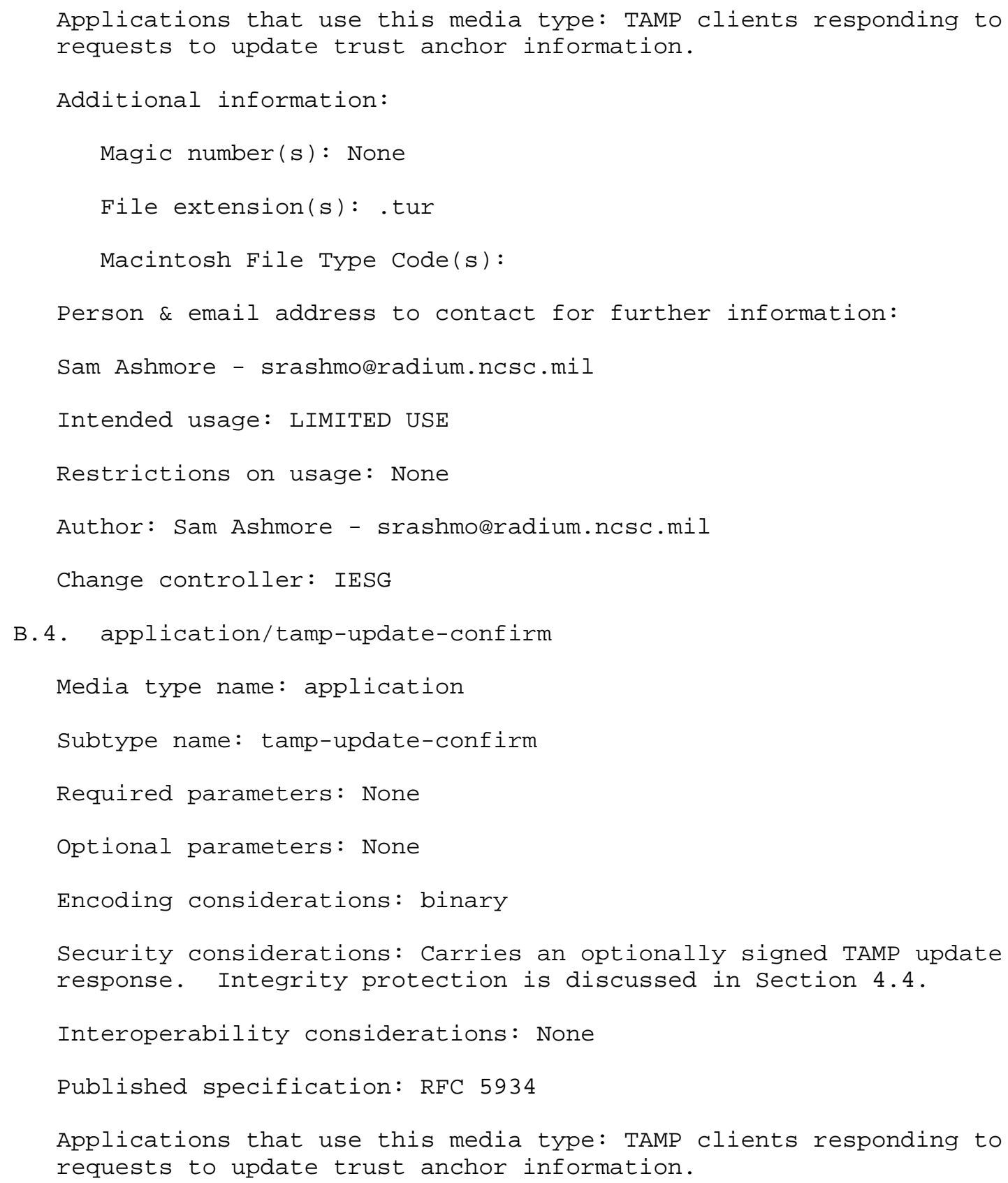




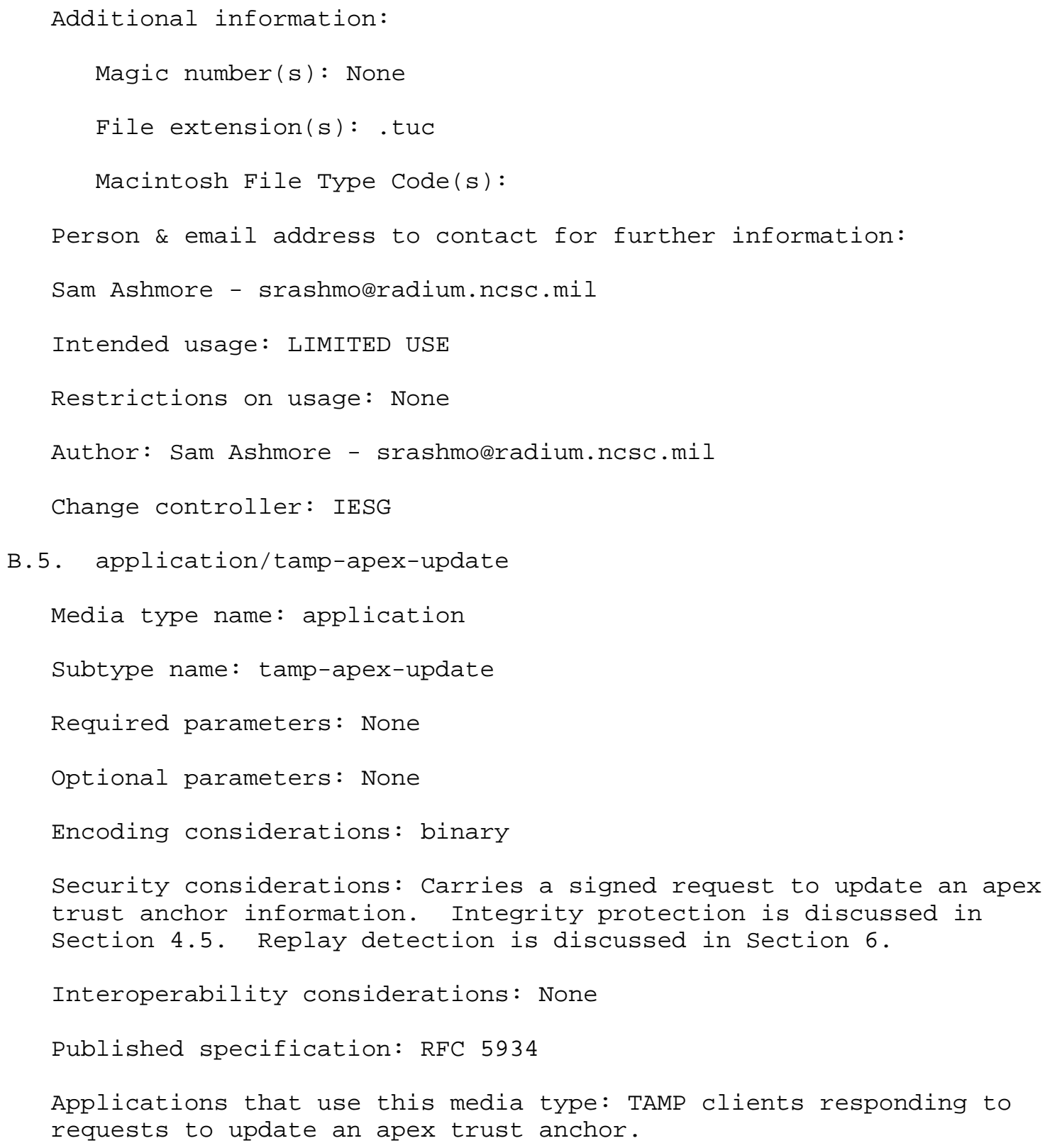




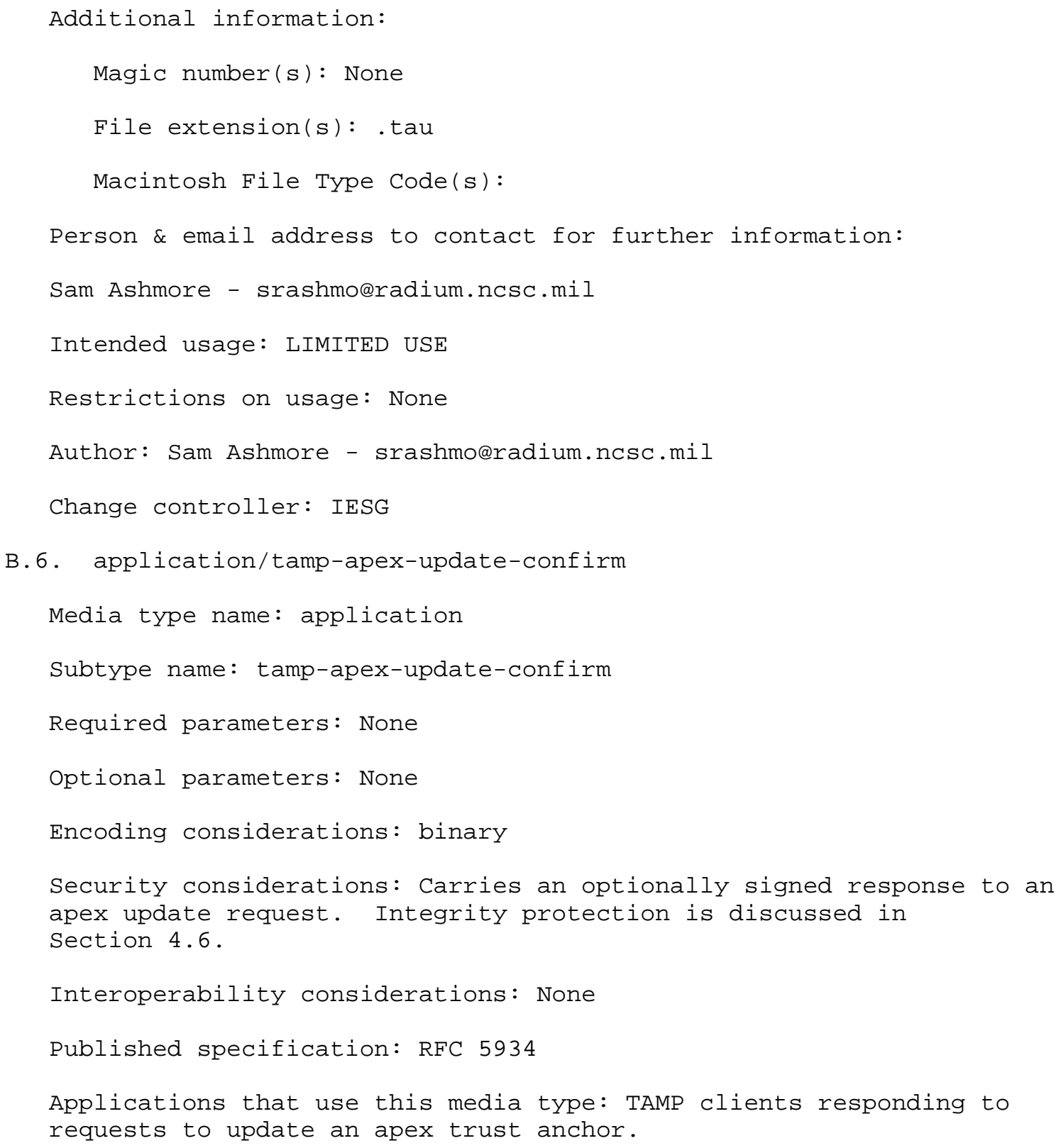




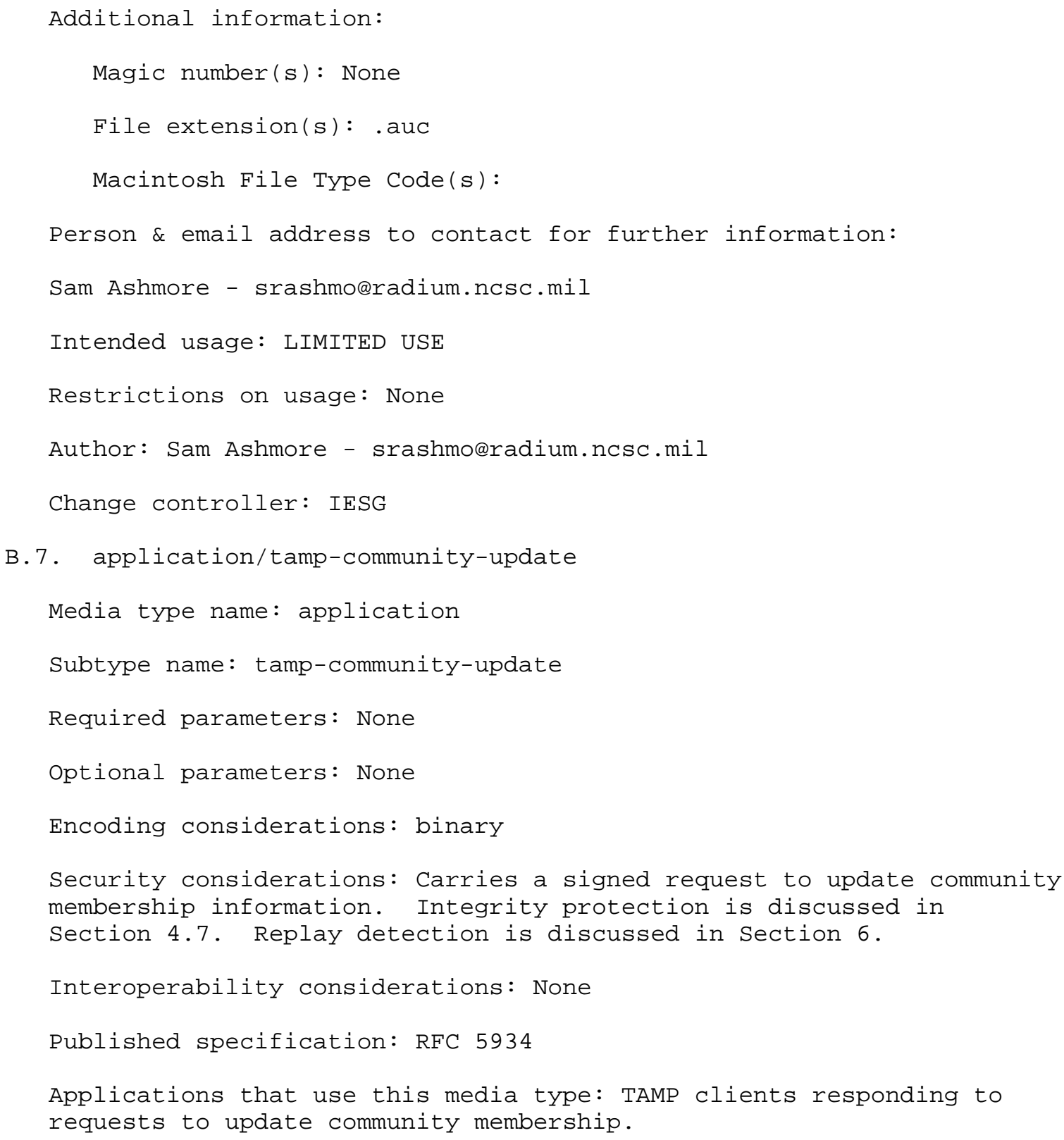




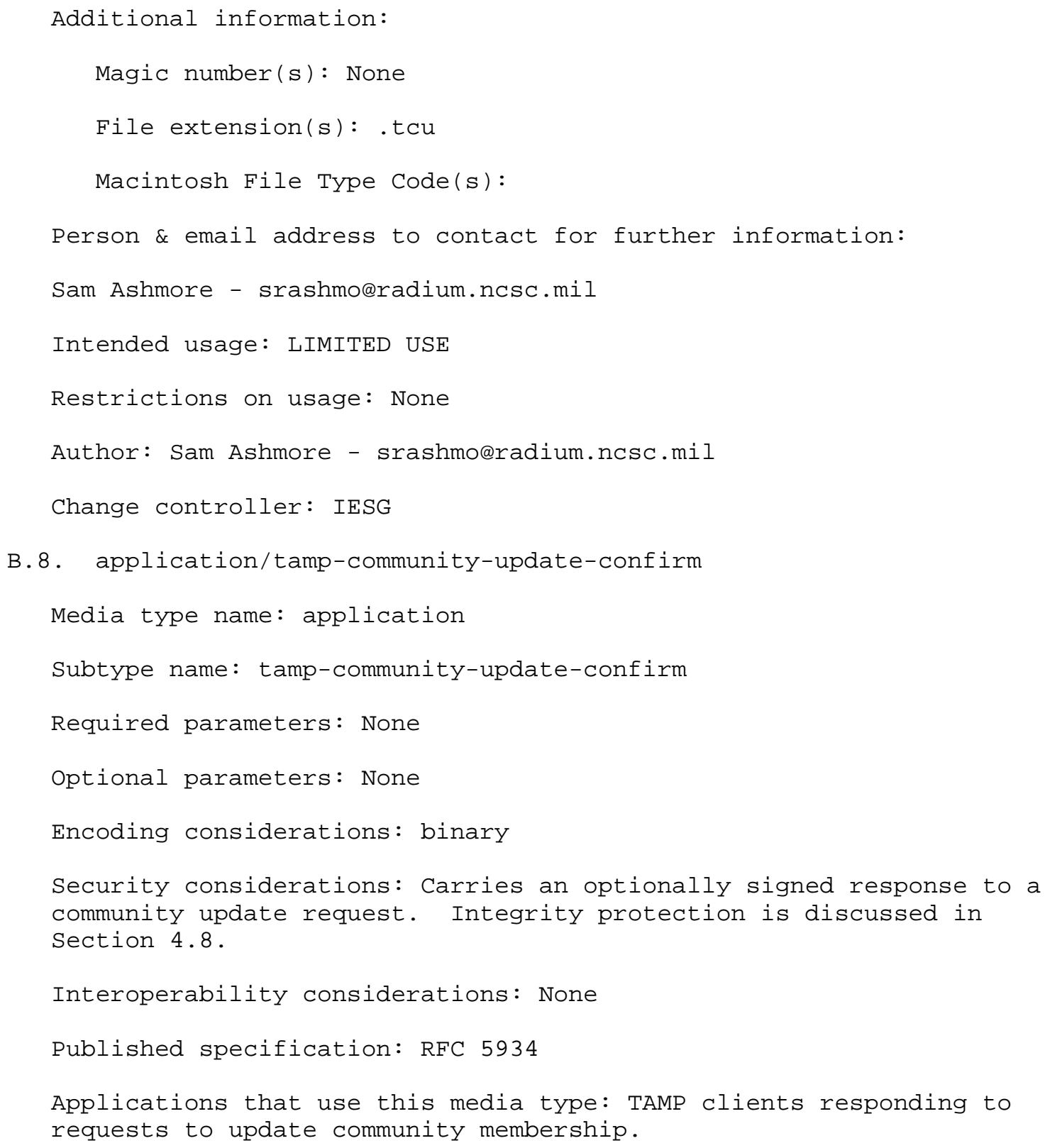




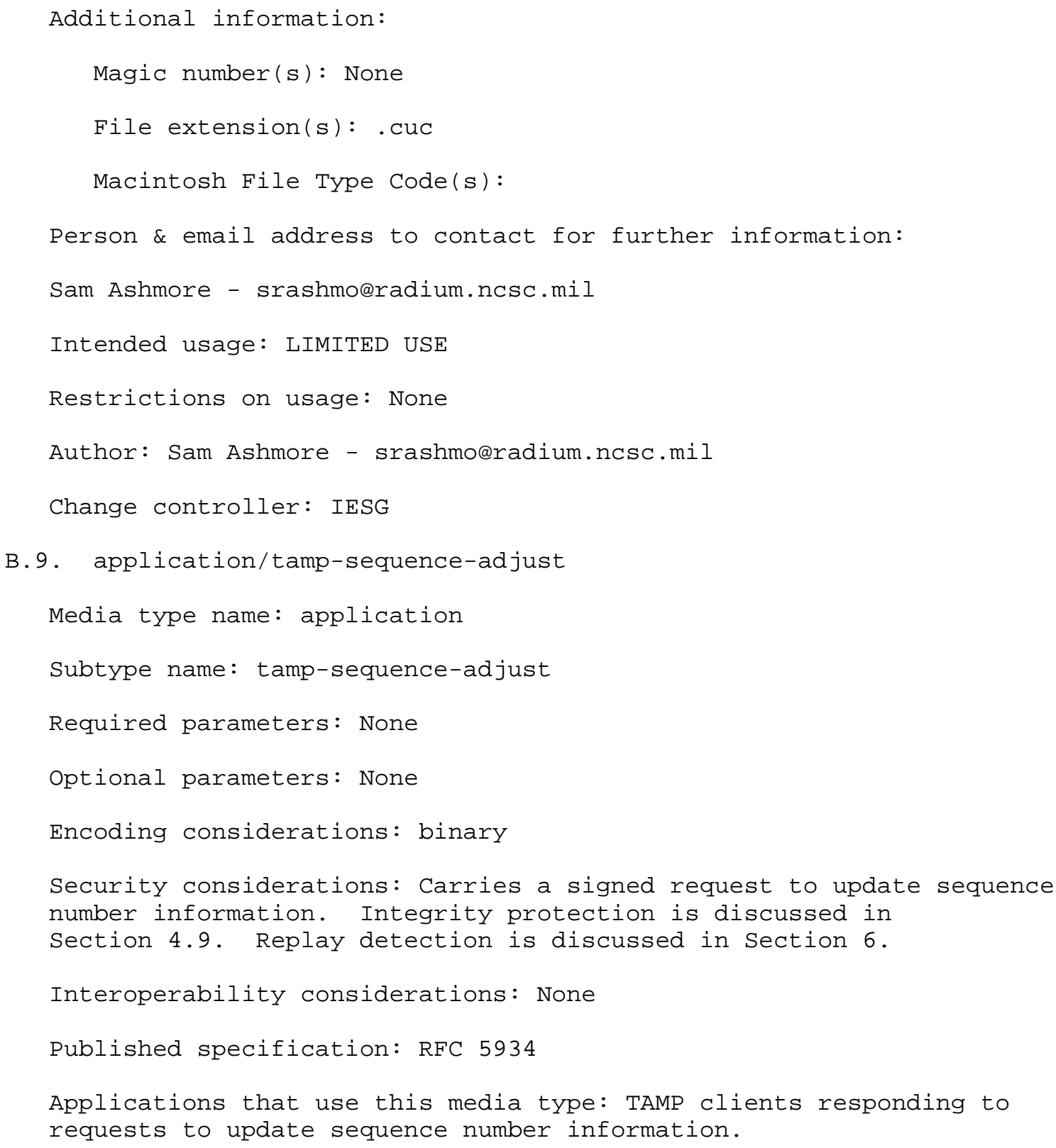




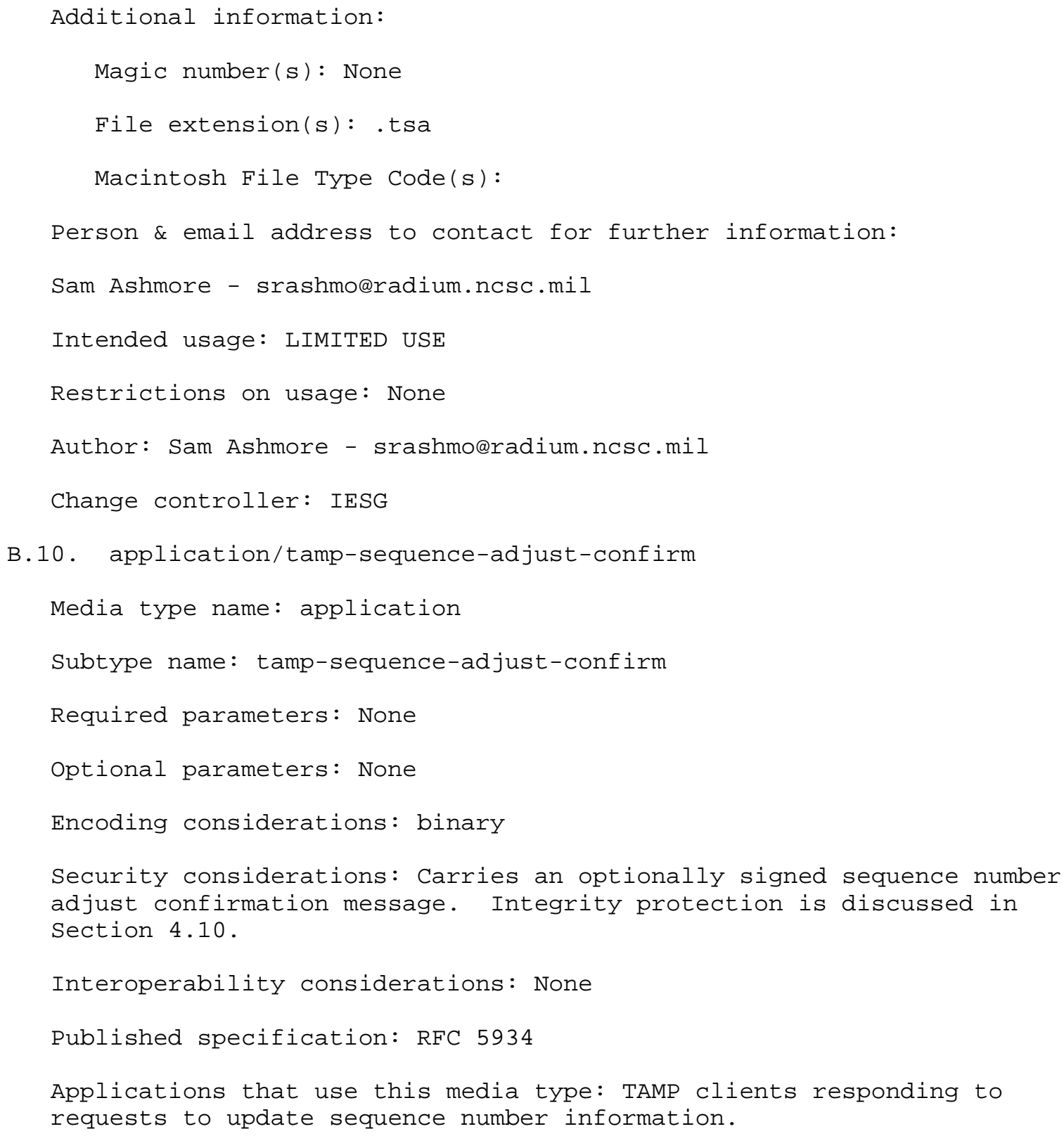




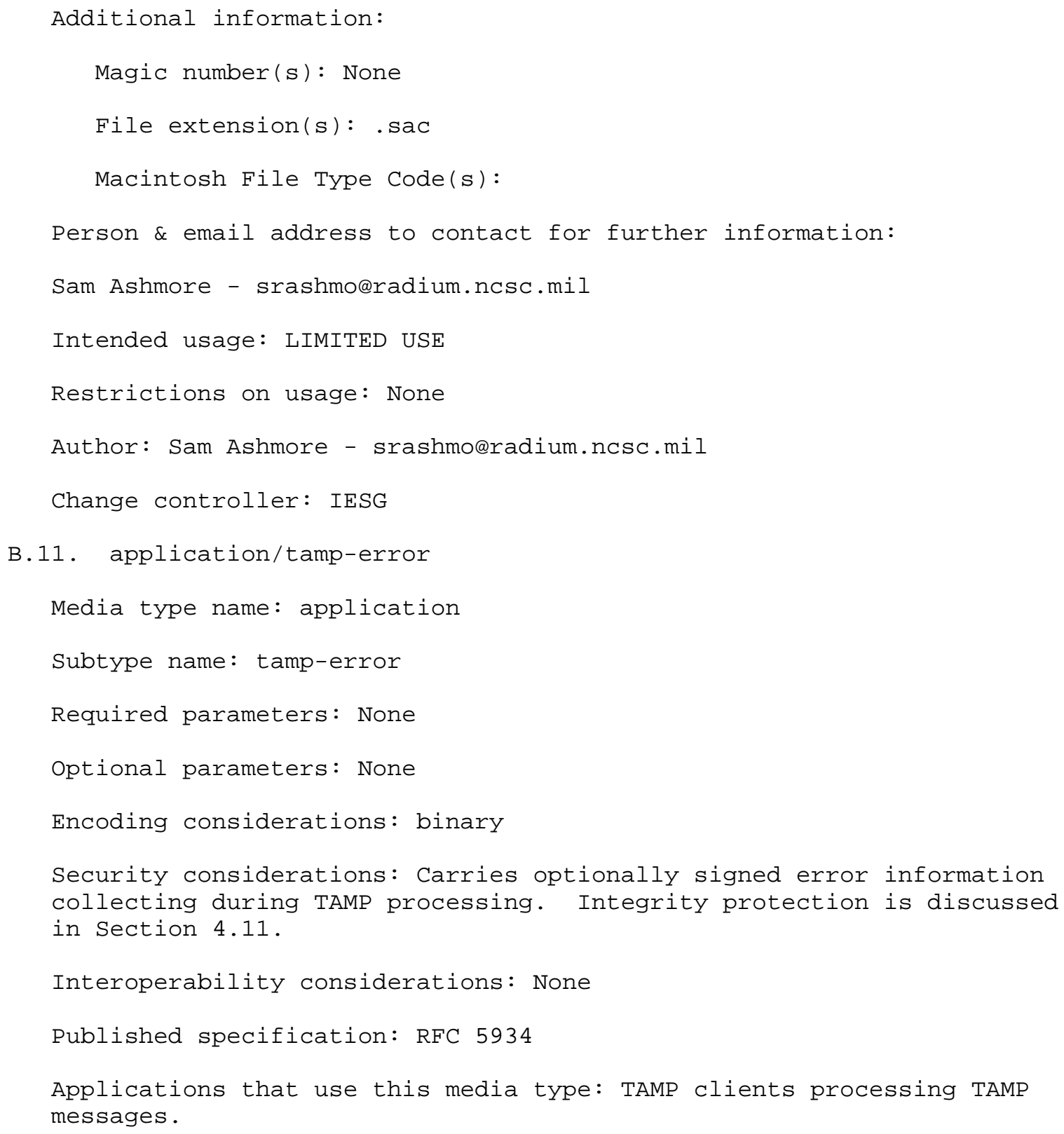


Additional information:

Magic number $(s)$ : None

File extension(s): .ter

Macintosh File Type Code(s):

Person \& email address to contact for further information:

Sam Ashmore - srashmo@radium.ncsc.mil

Intended usage: LIMITED USE

Restrictions on usage: None

Author: Sam Ashmore - srashmolradium.ncsc.mil

Change controller: IESG

Appendix C. TAMP over HTTP

This appendix describes the formatting and transportation conventions for the TAMP messages when carried by HTTP [RFC2616]. Each TAMP message type is covered by a subsection below. Each TAMP request message sent via HTTP is responded to either with an HTTP response containing a TAMP response or error or, if failure occurs prior to invoking TAMP, an HTTP error. TAMP response, confirmation, and error messages are not suitable for caching. In order for TAMP clients and servers using HTTP to interoperate, the following rules apply.

- Clients MUST use the POST method to submit their requests.

- Servers MUST use the 200 response code for successful responses.

- Clients MAY attempt to send HTTPS requests using Transport Layer Security (TLS) 1.0 or later, although servers are not required to support TLS.

- Servers MUST NOT assume client support for any type of HTTP authentication such as cookies, Basic authentication, or Digest authentication.

- Clients and servers are expected to follow the other rules and restrictions in [RFC2616]. Note that some of those rules are for HTTP methods other than POST; clearly, only the rules that apply to POST are relevant for this specification. 


\section{C.1. TAMP Status Query Message}

A TAMP Status Query Message using the POST method is constructed as follows: The Content-Type header MUST have the value "application/ tamp-status-query" .

The body of the message is the binary value of the DER encoding of the TAMPStatusquery, wrapped in a CMS body as described in section 2 .

\section{C.2. TAMP Status Response Message}

An HTTP-based TAMP Status Response message is composed of the appropriate HTTP headers, followed by the binary value of the DER encoding of the TAMPStatusResponse, wrapped in a CMS body as described in Section 2 .

The Content-Type header MUST have the value "application/ tamp-status-response."

C.3. Trust Anchor Update Message

A Trust Anchor Update Message using the POST method is constructed as follows: The Content-Type header MUST have the value "application/ tamp-update".

The body of the message is the binary value of the DER encoding of the TAMPUpdate, wrapped in a CMS body as described in section 2 .

C.4. Trust Anchor Update Confirm Message

An HTTP-based Trust Anchor Update Confirm message is composed of the appropriate HTTP headers, followed by the binary value of the DER encoding of the TAMPUpdateConfirm, wrapped in a CMS body as described in Section 2 .

The Content-Type header MUST have the value "application/ tamp-update-confirm".

\section{C.5. Apex Trust Anchor Update Message}

An Apex Trust Anchor Update Message using the POST method is constructed as follows: The Content-Type header MUST have the value "application/tamp-apex-update".

The body of the message is the binary value of the DER encoding of the TAMPApexUpdate, wrapped in a CMS body as described in Section 2. 


\section{C.6. Apex Trust Anchor Update Confirm Message}

An HTTP-based Apex Trust Anchor Update Confirm message is composed of the appropriate HTTP headers, followed by the binary value of the DER encoding of the TAMPApexUpdateConfirm, wrapped in a CMS body as described in section 2.

The Content-Type header MUST have the value "application/ tamp-apex-update-confirm".

C.7. Community Update Message

A Community Update Message using the POST method is constructed as follows: The Content-Type header MUST have the value "application/ tamp-community-update".

The body of the message is the binary value of the DER encoding of the TAMPCommunityUpdate, wrapped in a CMS body as described in Section 2 .

\section{C.8. Community Update Confirm Message}

An HTTP-based Community Update Confirm message is composed of the appropriate HTTP headers, followed by the binary value of the DER encoding of the TAMPCommunityUpdateConfirm, wrapped in a CMS body as described in section 2.

The Content-Type header MUST have the value "application/ tamp-community-update-confirm" .

\section{C.9. Sequence Number Adjust Message}

A Sequence Number Adjust Message using the POST method is constructed as follows: The Content-Type header MUST have the value "application/ tamp-sequence-adjust" .

The body of the message is the binary value of the DER encoding of the sequenceNumberAdjust, wrapped in a CMS body as described in Section 2 .

\section{C.10. Sequence Number Adjust Confirm Message}

An HTTP-based Sequence Number Adjust Confirm message is composed of the appropriate HTTP headers, followed by the binary value of the DER encoding of the SequenceNumberAdjustConfirm, wrapped in a CMS body as described in section 2. 
The Content-Type header MUST have the value "application/ tamp-sequence-adjust-confirm" .

\section{C.11. TAMP Error Message}

An HTTP-based TAMP Error message is composed of the appropriate HTTP headers, followed by the binary value of the DER encoding of the TAMPError, wrapped in a CMS body as described in section 2 .

The Content-Type header MUST have the value "application/tamp-error". Authors' Addresses

Russ Housley

Vigil Security, LLC

918 Spring Knoll Drive

Herndon, VA 20170

USA

EMail: housley@vigilsec.com

Sam Ashmore

National Security Agency

Suite 6751

9800 Savage Road

Fort Meade, MD 20755

USA

EMail: srashmo@radium.ncsc.mil

Carl Wallace

Cygnacom Solutions

Suite 5400

7925 Jones Branch Drive

McLean, VA 22102

USA

EMail: cwallacedcygnacom.com 